Florida International University FIU Digital Commons

FIU Electronic Theses and Dissertations

University Graduate School

9-26-2017

\title{
Epigenetic Instability Induced by DNA Base Lesion via DNA Base Excision Repair
}

Zhongliang Jiang

Florida International University, zjian005@fiu.edu

DOI: 10.25148 /etd.FIDC003999

Follow this and additional works at: https://digitalcommons.fiu.edu/etd

Part of the Amino Acids, Peptides, and Proteins Commons, Biochemistry Commons, Enzymes and Coenzymes Commons, Nucleic Acids, Nucleotides, and Nucleosides Commons, and the Other Pharmacology, Toxicology and Environmental Health Commons

\section{Recommended Citation}

Jiang, Zhongliang, "Epigenetic Instability Induced by DNA Base Lesion via DNA Base Excision Repair" (2017). FIU Electronic Theses and Dissertations. 3566.

https://digitalcommons.fiu.edu/etd/3566

This work is brought to you for free and open access by the University Graduate School at FIU Digital Commons. It has been accepted for inclusion in FIU Electronic Theses and Dissertations by an authorized administrator of FIU Digital Commons. For more information, please contact dcc@fiu.edu. 


\section{FLORIDA INTERNATIONAL UNIVERSITY \\ Miami, Florida}

\section{EPIGENETIC INSTABILITY INDUCED BY DNA BASE LESIONS VIA DNA BASE EXCISION REPAIR}

A dissertation submitted in partial fulfillment of

the requirements for the degree of

DOCTOR OF PHILOSOPHY

in

BIOCHEMISTRY

by

Zhongliang Jiang 
To: Dean Michael R. Heithaus

College of Arts, Sciences and Education

This dissertation, written by Zhongliang Jiang, and entitled Epigenetic Instability Induced by DNA Base Lesions via DNA Base Excision Repair, having been approved in respect to style and intellectual content, is referred to you for judgment.

We have read this dissertation and recommend that it be approved.

Irina Agoulnik

Lou Kim

Yuk-Ching Tse-Dinh

Xiaotang Wang

Yuan Liu, Major Professor

Date of Defense: September 26, 2017

The dissertation of Zhongliang Jiang is approved.

Dean Michael R. Heithaus

College of Arts, Sciences and Education

Andrés G. Gil

Vice President for Research and Economic Development and Dean of the University Graduate School

Florida International University, 2017 
C Copyright 2017 by Zhongliang Jiang

All rights reserved. 


\section{DEDICATION}

I dedicate this dissertation to my parents, Ying Li and Fengming Jiang, for their love, support, and encouragement. 


\section{ACKNOWLEDGMENTS}

I would like to thank my advisor and all the members in the Liu Laboratory who have helped me and given me support throughout the completion of my $\mathrm{Ph} . \mathrm{D}$. research and training. First of all, I would like to thank my advisor, Dr. Yuan Liu, for her unending assistance, advice, and support. Under her excellent mentorship and guidance, I have gained invaluable experience in scientific research, writing, and presentation. She has encouraged and supported me every step of the way.

I would like to thank my committee members, Dr. Irina Agoulnik, Dr. Lou Kim, Dr. Yuk-Ching Tse-Dinh, and Dr. Xiaotang Wang, for their advice, guidance, and support throughout my Ph.D. training and career development.

I would like to thank Dr. Yanhao Lai, who has been providing great assistance and guidance for my research on almost each step. She has offered so much help and support throughout my research training. I would also like to thank Dr. Meng $\mathrm{Xu}$, Dr. Jill Beaver, Yaou Ren, Ruipeng Lei, Eduardo Laverde, and Shantell Rolle, as well as all other past and present members of the Liu Laboratory for their great help and support.

Finally, I thank the FIU Dissertation Year Fellowship (DYF) which provided me with great support for me to finish the last part of my graduate research. 


\section{ABSTRACT OF THE DISSERTATION \\ EPIGENETIC INSTABILITY INDUCED BY DNA BASE LESIONS VIA DNA BASE \\ EXCISION REPAIR}

by

Zhongliang Jiang

Florida International University, 2017

Miami, Florida

\section{Professor Yuan Liu, Major Professor}

DNA damage can cause genome instability, which may lead to human cancer. The most common form of DNA damage is DNA base damage, which is efficiently repaired by DNA base excision repair (BER). Thus BER is the major DNA repair pathway that maintains the stability of the genome. On the other hand, BER mediates DNA demethylation that can occur on the promoter region of important tumor suppressor genes such as Breast Cancer 1 (BRCA1) gene that is also involved in prevention and development of cancer. In this study, employing cell-based and in vitro biochemical approaches along with bisulfite DNA sequencing, we initially discovered that an oxidized nucleotide, 5',2cyclo-2-deoxyadenosine in DNA duplex can either cause misinsertion by DNA polymerase $\beta$ (pol $\beta$ ) during pol $\beta$-mediated BER or inhibit lesion bypass of pol $\beta$ resulting in DNA strand breaks. We then explored how a $\mathrm{T} / \mathrm{G}$ mismatch resulting from active DNA demethylation can affect genome integrity during BER and found that pol $\beta$ can extend the mismatched T to cause mutation. We found that AP endonuclease 1 (APE1) can use its 3'$5^{\prime}$ exonuclease to remove the mismatched $\mathrm{T}$ before pol $\beta$ can extend the nucleotide preventing a $\mathrm{C}$ to $\mathrm{T}$ mutation. The results demonstrate that the $3^{\prime}-5^{\prime}$ exonuclease activity of 
APE1 can serve as a proofreader for pol $\beta$ to prevent mutation. We further explored the effects of exposure of environmental toxicants, bromate and chromate on the DNA methylation pattern on the promoter region of BRCA1 gene with bisulfite DNA sequencing. We found that bromate and chromate induced demethylation of 5methylcytosines $(5 \mathrm{mC})$ at the $\mathrm{CpG}$ sites as well as created additional methylation at several unmethylated CpG sites at BRCA1 gene in human embryonic kidney (HEK) 293 cells. We further demonstrated that the demethylation was mediated by pol $\beta$ nucleotide misinsertion and an interaction between pol $\beta$ and DNA methyltransferase 1 (DNMT1) suggesting a cross-talk between BER and DNA methyltransferases. We suggest that DNA base damage and BER govern the interactions among the environment, the genome and epigenome, modulating the stability of the genome and epigenome and disease development. 


\section{TABLE OF CONTENTS}

CHAPTER

PAGE

INTRODUCTION

\section{1}

A. DNA DAMAGE CAUSED BY ENVIRONMENTAL TOXICANTS, BROMATE AND CHROMATE

B. BASE EXCISION REPAIR IS THE MAJOR PATHWAY TO REPAIR OXIDATIVE DNA DAMAGE AND PLAYS A CRITICAL ROLE IN ACTIVE DEMETHYLATION IN CpG DINUCLEOTIDE CLUSTERS .11 C. DNA DAMAGE DISRUPTS DNA METHYLATION PATTERN BY MODULATING ACTIVITIES OF DNA METHYLTRANSFERASES (DNMTs). .18

D. BREAST CANCER 1 (BRCA1) PLAYS A CRITICAL ROLE IN PREVENTING

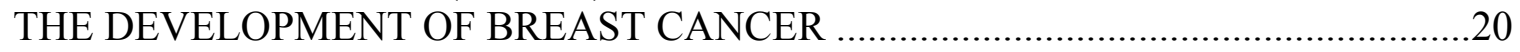
OVERVIEW

CHAPTER 1: BYPASS OF A 5',8-CYCLOPURINE-2'DEOXYNUCLEOSIDE BY DNA POLYMERASE $\beta$ DURING REPLICATION AND BASE EXCISION REPAIR LEADS TO NUCLEOTIDE MISINSERTIONS AND DNA STRAND BREAKS

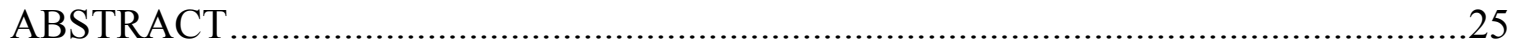

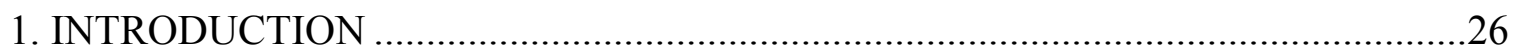

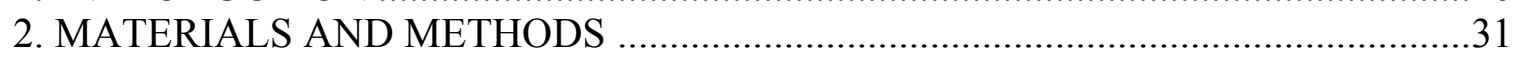

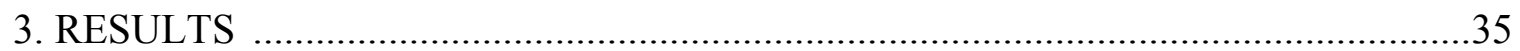

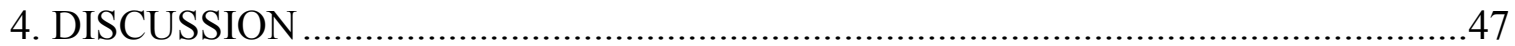

CHAPTER 2: AP ENDONUCLEASE 1 COMBATS THE EXTENSION OF A T/G MISMATCH BY DNA POLYMERASE $\beta$ TO PREVENT MUTATIONS IN CpGs DURING BASE EXCISION REPAIR

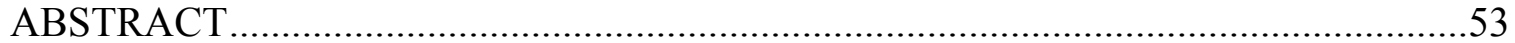

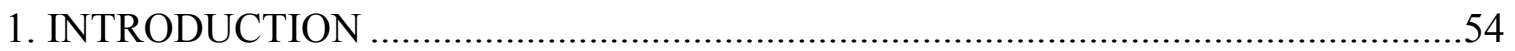

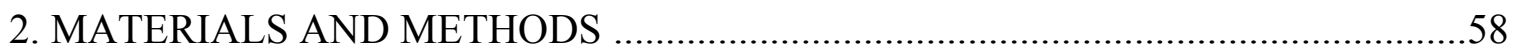

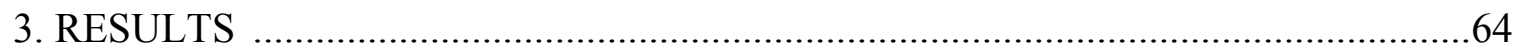

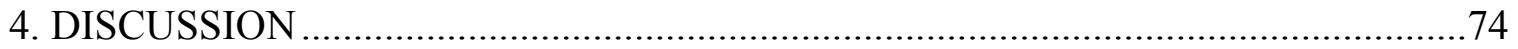

CHAPTER 3: ENVIRONMENTALLY-INDUCED OXIDATIVE DNA DAMAGE DISRUPTS DNA METHYLATION PATTERN IN HUMAN BREAST CANCER 1 (BRCA1) GENE VIA BASE EXCISION REPAIR ABSTRACT

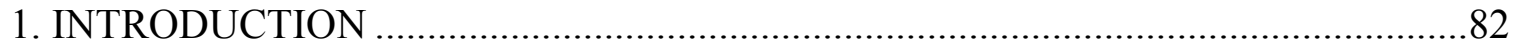

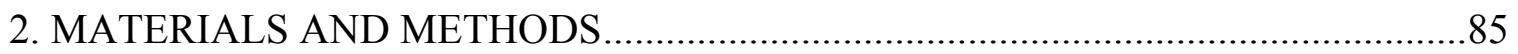

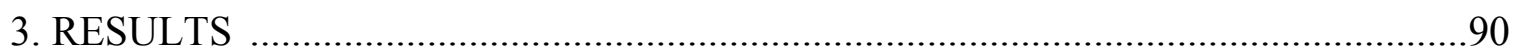

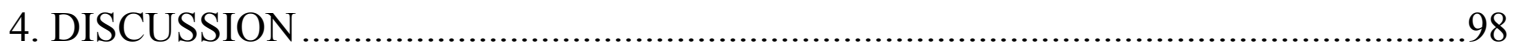




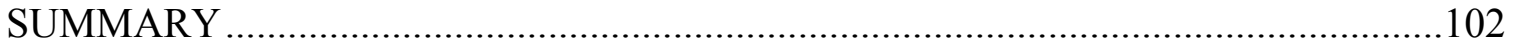

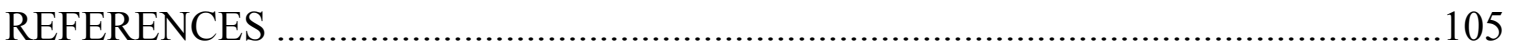

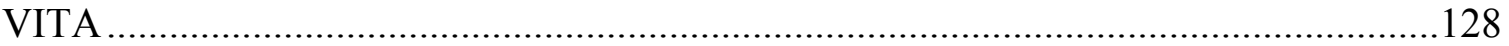




\section{LIST OF FIGURES}

FIGURE

PAGE

\section{INTRODUCTION}

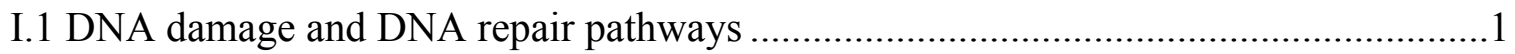

I.2 FPG removes the DNA damage induced by $\mathrm{KBrO} 3$...............................................

I.3 A possible mechanism of guanine oxidation induced by $\mathrm{KBrO} 3$ in the presence of

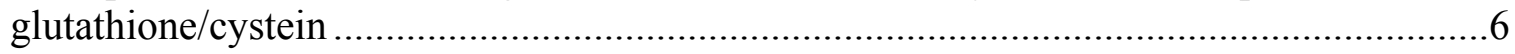

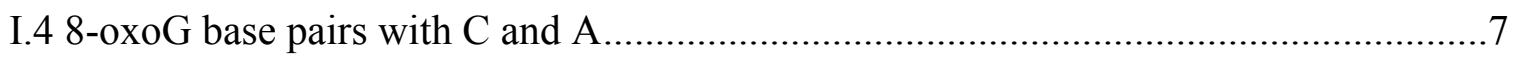

I.5 Major steps in uptake, metabolism and formation of DNA damage by $\mathrm{Cr}(\mathrm{VI})$...........9

I.6 Pathways of DNA Base Excision Repair ........................................................ 12

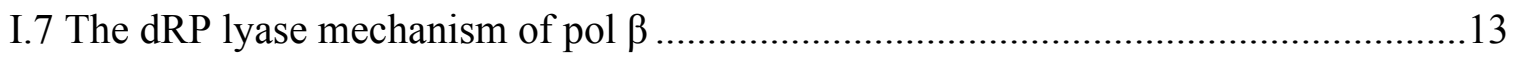

I. 8 Cytosine methylation and pathways of active DNA demethylation..........................15

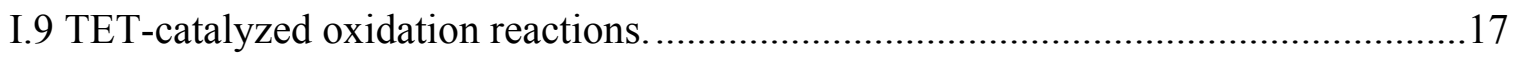

I.10 BRCA1 gene is located on the 17th chromosome in human genome ......................20

\section{CHAPTER 1}

1.1 The structures of 5',8-cyclo-2'-deoxyadenosines

1.2 Pol $\beta$ plays an important role in bypassing a cdA lesion during DNA replication and BER

1.3 Pol $\beta$ DNA synthesis with a template cdA lesion during DNA replication and BER .39

1.4 FEN1 flap cleavage during pol $\beta$ bypass of a cdA

1.5 Completion of DNA lagging strand maturation and BER through pol $\beta$ bypass of a cdA lesion.

1.6 Pol $\beta$ nucleotide insertions in bypassing a cdA lesion during DNA replication and BER 
1.7 Pol $\beta$ bypass of a cdA lesion leads to nucleotide misinsertion and DNA strand breaks during DNA replication and BER

\section{CHAPTER 2}

2.1 The activity of TDG in removing a T/G mismatch at a $\mathrm{CpG}$ dinucleotide is inhibited by an adjacent abasic lesion

2.2 APE1 can efficiently incise an abasic site that is adjacent to a $\mathrm{T} / \mathrm{G}$ mismatch in a $\mathrm{CpG}$ dinucleotide in the presence of TDG.

2.3 Pol $\beta$ extends a 3'-terminus mismatched $\mathrm{T}$ at a $\mathrm{CpG}$ dinucleotide.

2.4 APE1 3'-5'exonuclease activity removes a 3'-terminus mismatched $\mathrm{T}$ in a $\mathrm{CpG}$ dinucleotide.

2.5 FEN1 flap cleavage in the presence of a $\mathrm{T} / \mathrm{G}$ mismatch in a $\mathrm{CpG}$ dinucleotide

2.6 The activities of pol $\beta$ DNA synthesis and APE1 3' -5 ' exonuclease in the context of a $\mathrm{T} / \mathrm{G}$ mismatch in a $\mathrm{CpG}$ dinucleotide

2.7 BER reconstitution in the context of a $\mathrm{T} / \mathrm{G}$ mismatch in a $\mathrm{CpG}$ dinucleotide

2.8 APE1 3' -5 ' exonuclease combats the extension of a T/G mismatch by pol $\beta$ and $\mathrm{C}$ to $\mathrm{T}$ mutations in CpGs during BER..

\section{CHAPTER 3}

3.1 The DNA methylation pattern on BRCA1 promoter from -189 to +27 in HEK293 cells with and without bromate and chromate treatment

3.2 The DNA methylation pattern on BRCA1 gene promoter and encoding region from -189 to +27 in HEK293 cells with or without treatment of bromate and chromate.

3.3 The mutation spectrum of BRCA1 gene in HEK293 cells induced by chromate (A) and bromate $(\mathrm{B})$ treatment.

3.4 HEK293 cells transfected with GFP-pol $\beta$ were treated with $10 \mu \mathrm{M}$ chromate for 1 hour. 
3.6 Chromate exposure resulted in the interaction between pol $\beta$ and DNMT1

3.7 The hypothetical model for bromate and chromate to alter DNA methylation pattern of the BRCA1 gene via DNA base lesions and BER in human cells 


\section{ABBREVIATIONS AND ACRONYMS}

${ }^{\circ} \mathrm{C}$

A

AAG

ADP

AID

APE1

AP site

APOBEC

ATM

ATP

ATR

BER

BRCA1

BRCC36

BRCC45

BRCTs

BSA

C

CCDC98/Abraxas

$\operatorname{cdA}$

$\mathrm{cdG}$ degree Celsius

adenine

3-alkyladenine DNA glycosylase

adenosine diphosphate

activation-induced cytidine deaminase

AP endonuclease 1

apurinic/apyrimidinic site

apolipoprotein B mRNA editing enzyme, catalytic polypeptide-like

ataxia telangiectasia mutated

adenosine triphosphate

ataxia telangiectasia and $\operatorname{rad} 3$ related

base excision repair

breast cancer gene 1

BRCA1/BRCA2-containing complex subunit 36

BRCA1/BRCA2-containing complex subunit 45

BRCA1-C-termus-domain containing proteins

bovine serum albumin

cytosine

coiled-coil domain-containing protein 98/abraxas protein

5',8-cyclo-2'-deoxyadenosine

5',8-cyclo-2'-deoxyguanosine 


\begin{tabular}{|c|c|}
\hline $\mathrm{CDC} 2$ & cell division cycle protein 2 homolog \\
\hline $\mathrm{CDC} 25$ & cell division cycle 25 homolog \\
\hline $\mathrm{cdPu}$ & 5',8-cyclo-2'-deoxypurines \\
\hline CFSs & common fragile sites \\
\hline Chk1 & checkpoint kinase 1 \\
\hline CLC & checkpoint loading complex \\
\hline CPDs & cyclobutane-pyrimidine dimers \\
\hline $\mathrm{CSC}$ & checkpoint sliding clamp \\
\hline DMEM & dulbecco's modified eagle's medium \\
\hline DMSO & dimethyl sulfoxide \\
\hline DNA & deoxyribonucleic acid \\
\hline dNTP & deoxyribonucleotide triphosphate \\
\hline $\mathrm{dRP}$ & deoxyribose phosphate \\
\hline DSB & double-strand break \\
\hline dsDNA & double-stranded DNA \\
\hline DTT & dithiothreitol \\
\hline 8-oxoG & 8-oxoguanine \\
\hline E. coli & Escherichia coli \\
\hline EDTA & ethylenediaminetetraacetic acid \\
\hline ERCFSs & early replication fragile sites \\
\hline 5',8-cdA & 5',8-cyclo-2'-deoxyadenosine \\
\hline $5^{\prime}-\mathrm{dRP}$ & 5'-deoxyribose phosphate \\
\hline
\end{tabular}




\begin{tabular}{|c|c|}
\hline $5 \mathrm{hmC}$ & 5-hydroxymethylcytosine \\
\hline $5 \mathrm{hmU}$ & 5-hydroxymethyluracil \\
\hline $5 \mathrm{mC}$ & 5-methylcytosine \\
\hline FEN1 & flap endonuclease 1 \\
\hline FPG & formamidopyrimidine-DNA glycosylase \\
\hline FPLC & fast protein liquid chromatography system \\
\hline G & guanine \\
\hline HEPES & 4-(2-hydroxyethyl)-1-piperazineethanesulfonic acid \\
\hline HL60 & human promyelocytic leukemia cells \\
\hline HP100 & human promyelocytic leukemia cells \\
\hline HR & homologous recombination \\
\hline$\bullet$ & hydroxyl radical \\
\hline IARC & international agency for research on cancer \\
\hline IPTG & isopropyl $\beta$-D-1-thiogalactopyranoside \\
\hline IR & ionizing radiation \\
\hline LB & lysogeny broth \\
\hline LIG I & DNA ligase I \\
\hline LIG III & DNA ligase III \\
\hline LP-BER & long patch-base excision repair \\
\hline MEF & mouse embryonic fibroblast \\
\hline $\mathrm{MgCl}_{2}$ & magnesium chloride \\
\hline MLH & MutL homologue \\
\hline
\end{tabular}




\begin{tabular}{ll} 
MMR & mismatch repair \\
MRN & Mre11-Rad50-Nbs1 complex \\
MSH & MutS homologue \\
N & Nitrogen \\
Nbs1 & nibrin \\
NER & nucleotide excision repair \\
NHEJ & nonhomologous end joining \\
NP-40 & nonidet P-40 \\
nt & nucleotide \\
OGG1 & 8-oxoguanine DNA glycosylase \\
PAGE & polyacrylamide gel electrophoresis \\
PARP1 & poly(ADP-ribose) polymerase 1 \\
PBS & phosphate buffered saline \\
PCNA & proliferating cell nuclear antigen \\
PDG & propano-2'-deoxyguanosine \\
PI3KK & PI3-kinase-like kinase \\
PIP & PCNA-interacting protein \\
PMSF & phenylmethylsulfonyl fluoride \\
pol $\beta$ & DNA polymerase $\beta$ \\
\hline DNA & DNA polymerase $\varepsilon$ \\
&
\end{tabular}




\begin{tabular}{|c|c|}
\hline RAD51 & DNA recombination/repair protein rad51 \\
\hline RAD3-RAD26 & reactive attachment disorder 3-reactive attachment disorder 26 receptor \\
\hline RAP80 & associated protein 80 \\
\hline RNA & ribonucleic acid \\
\hline ROS & reactive oxygen species \\
\hline SDS & sodium dodecyl sulfate \\
\hline SP-BER & short patch base excision repair \\
\hline ssDNA & single-strand DNA \\
\hline $\mathrm{T}$ & thymine \\
\hline TDG & thymine DNA glycosylase \\
\hline TET & ten eleven translocation protein \\
\hline THF & tetrahydrofuran \\
\hline $\mathrm{XRCC} 1$ & $\mathrm{X}$-ray repair cross-complementing protein 1 \\
\hline $\mathrm{U}$ & uracil \\
\hline UDG & uracil DNA glycosylase \\
\hline UNG & uracil DNA N-glycosylase \\
\hline UTR & untranslated region \\
\hline UV & ultraviolet \\
\hline
\end{tabular}




\section{INTRODUCTION}

\section{A. DNA DAMAGE CAUSED BY ENVIRONMENTAL TOXICANTS, BROMATE}

\section{AND CHROMATE}

Deoxyribonucleic acid (DNA) is recognized as the "life code" which carries all the genetic information of a cell in the form of genes, which are the blueprint that determines all the protein sequences, structures, and the biological functions in a specific organism. However, double-strand DNA in living cells constantly encounters endogenous and exogenous DNA damaging agents (Hoeijmakers, 2001). As a result, DNA lesions are generated throughout every cell's life cycle. It has been estimated that there are

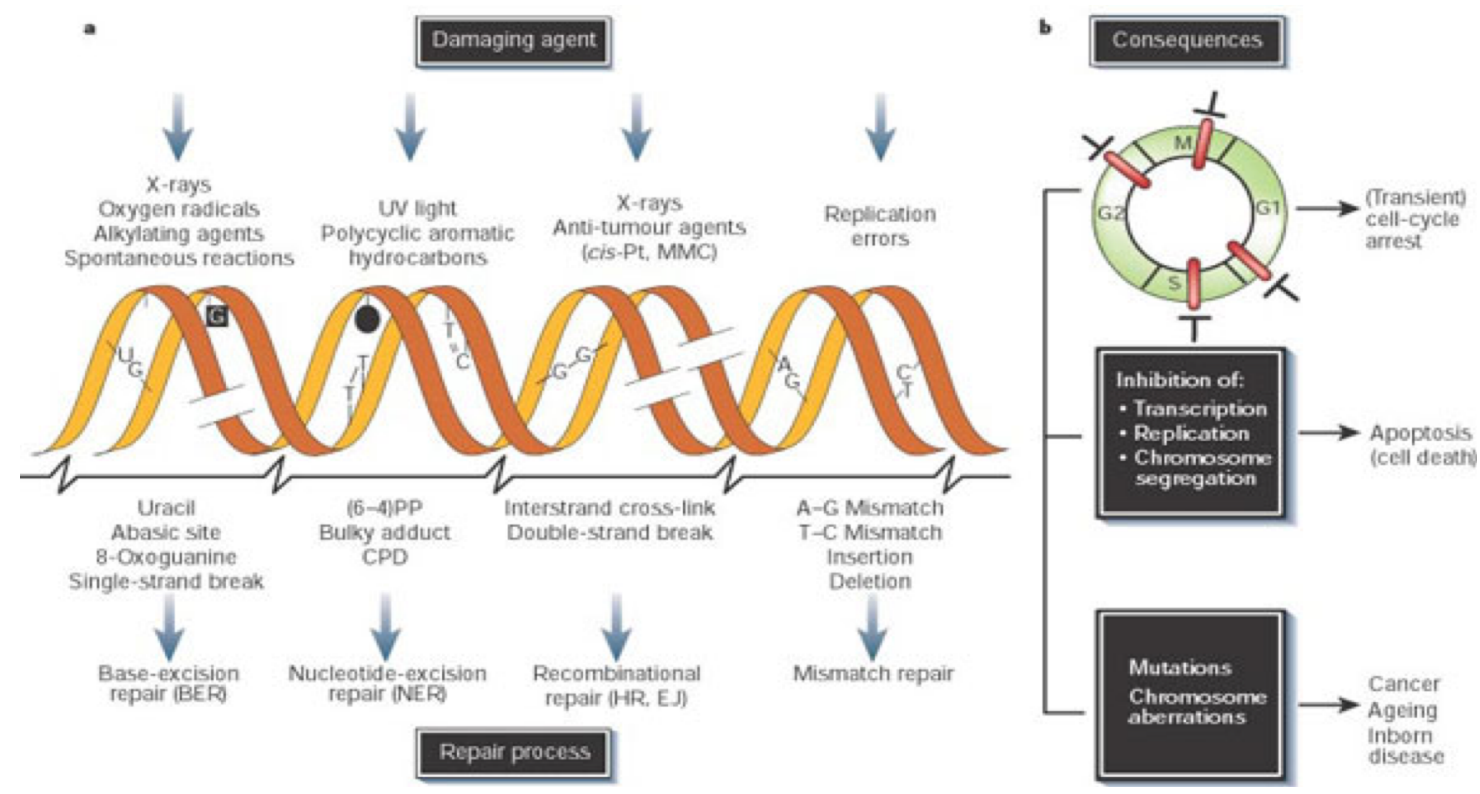

Figure I.1 DNA damage and DNA repair pathways. The DNA lesions induced by the DNA damaging agents are listed in the middle of Fig. I.1. DNA base lesions including uracil, abasic site, 8-oxoguanine (8-oxoG), and single-strand DNA (ssDNA) breaks are repaired by DNA base excision repair (BER). The bulky adducts, such as CPD (cyclobutane pyrimidine dimer) are repaired through nucleotide-excision repair (NER). The interstrand cross-links, double strand breaks are repaired through homologous recombination and nonhomologous end-joining (HR, NHEJ). The A-G, T-G mismatches, insertion, deletion errors are repaired by mismatch repair (Hoeijmakers, 2001). 
approximately 70,000 DNA lesions that are generated endogenously in a single human cell per day under normal physiological conditions (Lindahl and Barnes, 2000). Among them about $75 \%$ are single-strand DNA (ssDNA) breaks, which are produced through cell metabolism or spontaneous base hydrolysis which disrupts the molecule (Tubbs and Nussenzweig, 2017).

Another endogenous source of generating DNA damage is through replication and repair DNA polymerases. Since the human genome contains approximately 3 billion base pairs formed on the 23 chromosomes in the nucleus of each cell (Voet and Voet, 2011), which means that about $6 \times 10^{9}$ nucleotides are synthesized by DNA polymerases during DNA replication each time a cell divides. During DNA replication an error, can be made by nucleotide misinsertions of DNA polymerases at a low rate. Thus, a high frequency of cell divisions increases the probability of DNA replication errors by DNA polymerases which means the total number of cell divisions in normal stem cells derived from a specific type of tissue is usually used to assess the risk for cancer development in different tissues (Tomasetti and Vogelstein, 2015). Moreover, specific loci and DNA sequences in the genome also leads to susceptibility of DNA damage and mutations. For example, early replicating fragile sites (ERCFSs) that occur at the origins of replication in mammalian cells usually have highly expressed gene clusters enriched for repetitive sequences and CpG dinucleotides (Barlow et al., 2013) compared with late replicating common fragile sites (CFSs). These loci are hotspots of oncogene-induced DNA damage that preferentially attack the fragile sites (Sarni and Kerem, 2016).

Gene transcription can also form a source of endogenous DNA damage and plays a role in causing DNA damage and mutations. For example, transcription can induce the 
formation of an R-loop that in turn forms hotspots of endogenous DNA damage resulting from ROS and activation-induced cytidine deaminase (AID) leading to high frequencies of mutations and recombination (Tubbs and Nussenzweig, 2017). An increase in gene transcription stimulates spontaneous mutations in yeast (Datta and Jinks-Robertson, 1995). In mammalian cells, transcriptionally active gene loci bear more gene translocations and are more fragile than less-active loci (Barlow et al., 2013; Chiarle et al., 2011; Klein et al., 2011). A recent result from DNA sequencing of the genome of individual neurons in the human prefrontal cortex has shown that each neuron has more than 1,000 mutations that are induced by DNA damage in its own distinct genome (Lodato et al., 2015) which also appears to be a result of highly active transcriptional activity in human neurons. The mutations induced by DNA damage in neuron genomes further indicates that neuronal genome are constantly exposed to endogenous and exogenous DNA damage leading to mutations.

The exogenous DNA damaging sources include ionizing radiation, UV and a variety of environmental toxicants and pollutants that can induce DNA base lesions and DNA strand breaks. If not repaired, the damage can result in point mutations, recombination, genome instability, cell death and ultimately development of diseases including cancer and neurodegeneration. Early studies have shown that exposure to environmental pollutants can significantly increase the risk of cancer development. One well-known case is the high rate of skin carcinoma in chimney sweeps, which was first described in 1775 by Percivall Pott (Waldron, 1983b), and the soot exposure was confirmed as the cause of scrotal cancer in chimney sweeps (Waldron, 1983a). Another case was the experiment done in 1912 by Bayon who injected coal-tar into rabbit ears (Bayon, 1912) 
which resulted in coal tar-induced cancer. Katsusaburo Yamagiwa and Koichi Ichikawa repeated this experiment in 1915 by brushing coal-tar on to the ears of rabbits and also successfully induced cancer (Fujiki, 2014). These studies raised the awareness in the public that environmental pollutants play an important role in cancer development. Throughout many years of research, now we understand that DNA damage result from the exposure to both endogenous and exogenous sources plays a significant role in cancer development.

The environmental pollutant, potassium bromate $\left(\mathrm{KBrO}_{3}\right)$ had been a widely used as a food additive that was mainly added to flour as maturing agent to improve its texture in the bread-making process. Bromate was also widely used in beer malting and cheese making (Ahmad et al., 2014). However, later it was found that oral administration of bromate for 104 weeks induced renal cell tumor in F344 rats (Kurokawa et al., 1986) and was prohibited from being used as a food additive (IARC 1999). Nevertheless, bromate is currently still used in cold-wave hair lotions and textile dyeing with sulfur dyes (Ahmad et al., 2012; Ajarem et al., 2016; Khan et al., 2004; Kurokawa et al., 1986). Furthermore, because of the use of ozonation of drinking water, bromate has been generated as one of the major water disinfection byproducts (Campbell, 2006; Cavanagh, 1992; Dongmei et al., 2015). In vivo studies have shown that expose of human cell lines (HL-60 cells, HP100 cells) to bromate can significantly increased the cellular level of 8-oxo-7,8-dihydro-2'deoxyguanine (8-oxoG) in comparison with the non-treated cells (Murata et al., 2001). Employing an in vitro experiment, the Murata group found that bromate did not induce alkylated DNA bases or DNA backbone breakage (Figure I. 2A). Instead, bromate directly induced the formation of an 8-oxoG, which can be removed by formamidopyrimidineDNA glycosylase (FPG) (Figure I.2B). Since FPG can specifically remove an 8-oxoG, the 
results along with the previous results all indicate that bromate can induce the production of 8-oxoGs in living cells. A possible mechanism by which bromate generates 8-oxoGs in cells have been proposed and demonstrated in Figure I.3.

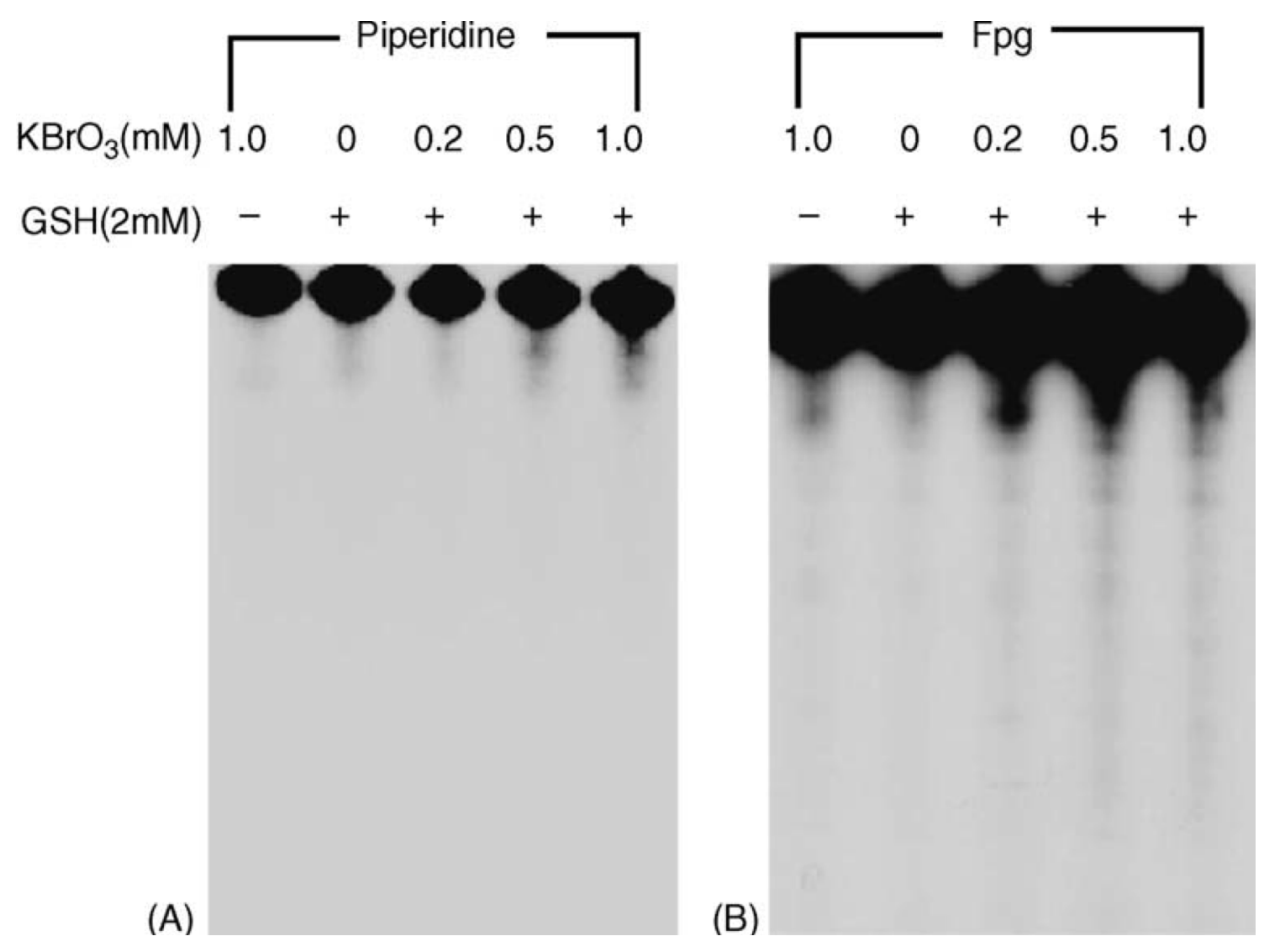

Figure I.2 FPG removes the DNA damage induced by $\mathrm{KBrO}_{3}$. DNA was treated with hot piperidine (A) or Fpg protein (B). Data are taken from (Murata et al., 2001) 


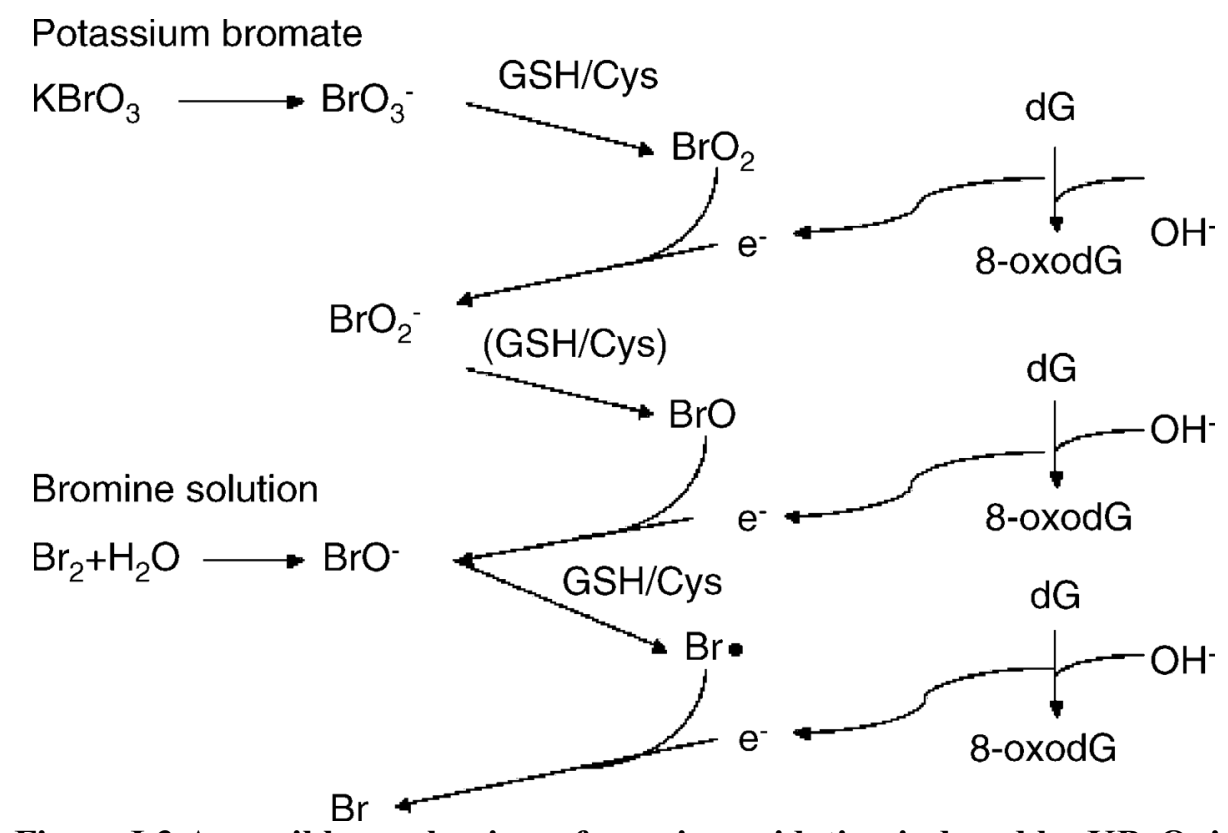

Figure I.3 A possible mechanism of guanine oxidation induced by $\mathrm{KBrO}_{3}$ in the presence of glutathione/cystein (Kawanishi and Murata, 2006).

As is shown in Figure I.3, guanine is the hotspot for bromate to generate the most prevalent DNA damage, 8-oxoGs. It was estimated that under physiological conditions, more than 10,000 8-oxoGs can be generated per cell per day. 8-oxoGs can be generated at a guanine at any location in the genome, and they can base pair with adenine through Hoogsteen base pair that results in mismatched base pairs and point mutations (Figure I.4) (David et al., 2007). Thus, mutations created by an 8-oxoG can happen at any site of any genes in the human genome. 

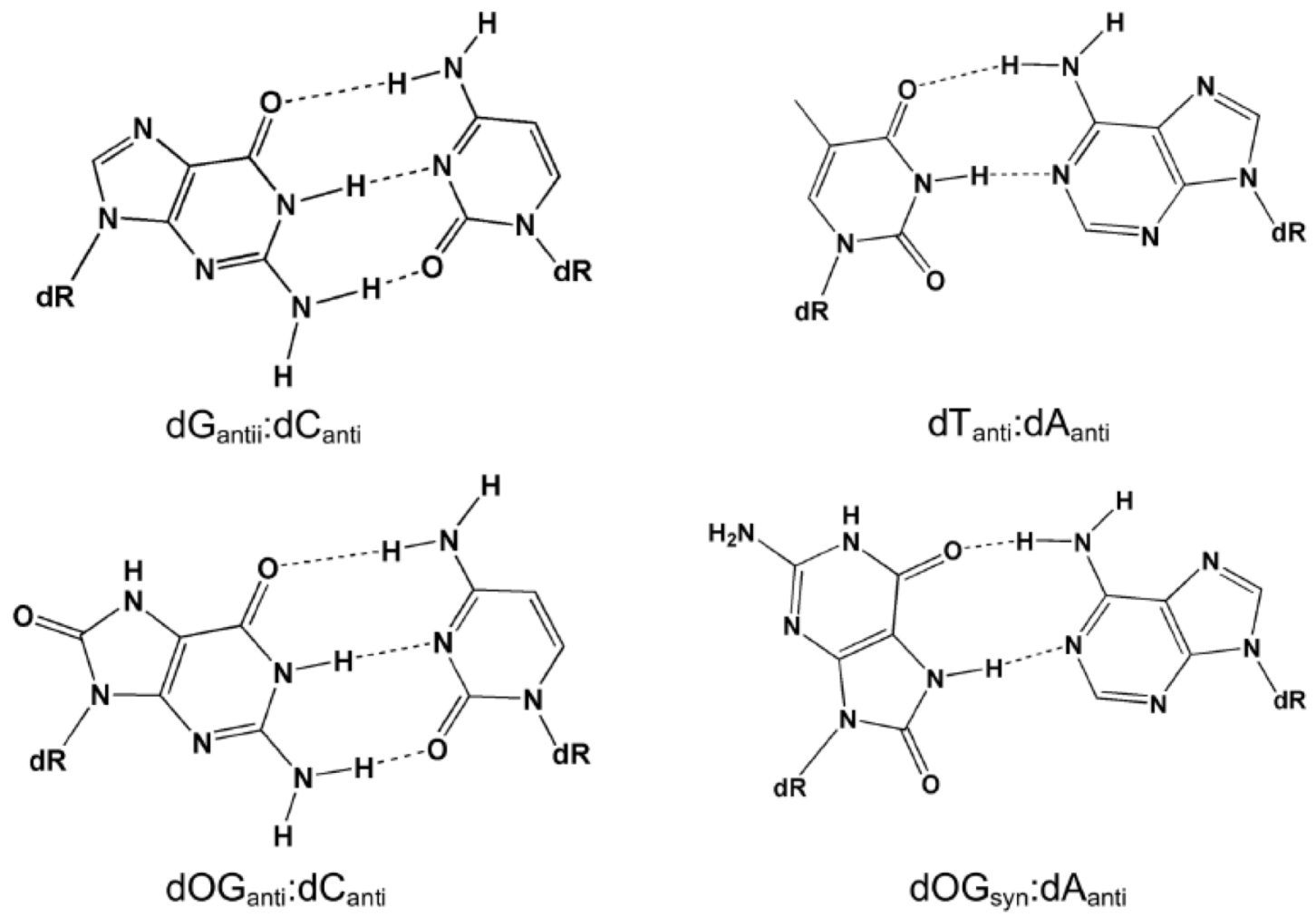

Figure I.4 8-oxoG base pairs with C and A (David et al., 2007)

Chromium (Cr) VI and its compounds including chromate. have been used in manufacturing of stainless steel and pressure-treated wood for years. About half a million industrial workers in the US and other countries undergo occupational exposure to $\mathrm{Cr}$ (VI) (Occupational and Health Administration, 2006). Chromium VI is a potent carcinogen and mutagen. The oxidation state of chromium has been found to be the key factor that determines the carcinogenicity and mutagenicity of various $\mathrm{Cr}$ (VI) compounds (Jennette, 1979). Under neutral $\mathrm{pH}, \mathrm{Cr}$ (VI) exists as a mixture of chromate $\left(\mathrm{CrO}_{4}{ }^{2-}\right)$ or hydrochromate $\left(\mathrm{HCrO}_{4}^{-}\right)$anions with the ratio around 3:1 (Zhitkovich, 2005). Chromium VI enters human cells readily through the sulfate channels (Alexander and Aaseth, 1995). In cells $\mathrm{Cr}$ (VI) can be reduced to $\mathrm{Cr}$ (III), the final oxidized form of $\mathrm{Cr}$ found in all 
biological systems which is thermodynamically stable. Animal studies have shown that all Cr (IV) compounds including calcium chromate, potassium chromate, lead chromate, chromic chromate, and strontium chromate, can result in sarcomas (spindle cell carcinomas and fibrosarcomas) in animals (Jennette, 1979). In contrast, Cr (III) compounds are not mutagenic. When $\mathrm{Cr}$ (VI) is reduced to Cr (III) extracellularly, it is usually considered as a detoxification $\mathrm{Cr}$ (VI) because of the poor cellular permeability of $\mathrm{Cr}$ (III) compounds. However, when Cr (VI) reduction occurs inside cells, it can exhibit genotoxicity (Salnikow and Zhitkovich, 2008). It has been shown that ascorbate is the main reducer of $\mathrm{Cr}$ (VI) in cells, particularly in cells that contain abundant ascorbate such as human lung cells (1.3 $\mathrm{mM})$. In cells with a low level of ascorbate such as human nonhepatic cells, thiols are responsible for reducing $\mathrm{Cr}(\mathrm{VI})$ (Zhitkovich, 2005). Although various amounts of $\mathrm{Cr}(\mathrm{V})$, $\mathrm{Cr}$ (IV) and organic chromium radicals can be generated during the Cr (VI) reduction process (Stearns and Wetterhahn, 1994), the end-product of its metabolism is always $\mathrm{Cr}$ (III), which forms complexes with proteins and nucleic acids.

The DNA damage generated during Cr (VI) reduction includes Cr-DNA adducts, DNA-protein crosslinks, DNA interstrand crosslinks, DNA breaks, and DNA base damage (Salnikow and Zhitkovich, 2008). Adducts of the Cr-DNA are the most abundant form of DNA lesions. They are induced by $\mathrm{Cr}(\mathrm{VI})$ reduction mediated by cysteine and ascorbate in mammalian cells (Figure I.5) (Salnikow and Zhitkovich, 2008). The reduction products include glutathione-Cr-DNA, cysteine-Cr-DNA, histidine-Cr-DNA and ascorbate-CrDNA complexes (Quievryn et al., 2002; Voitkun et al., 1994; Zhitkovich et al., 1995). It has been shown that acorbate-Cr-DNA crosslinks are the major pre-mutagenic Cr-DNA 
modifications (Quievryn et al., 2003; Voitkun et al., 1998). Chromate causes elevation of

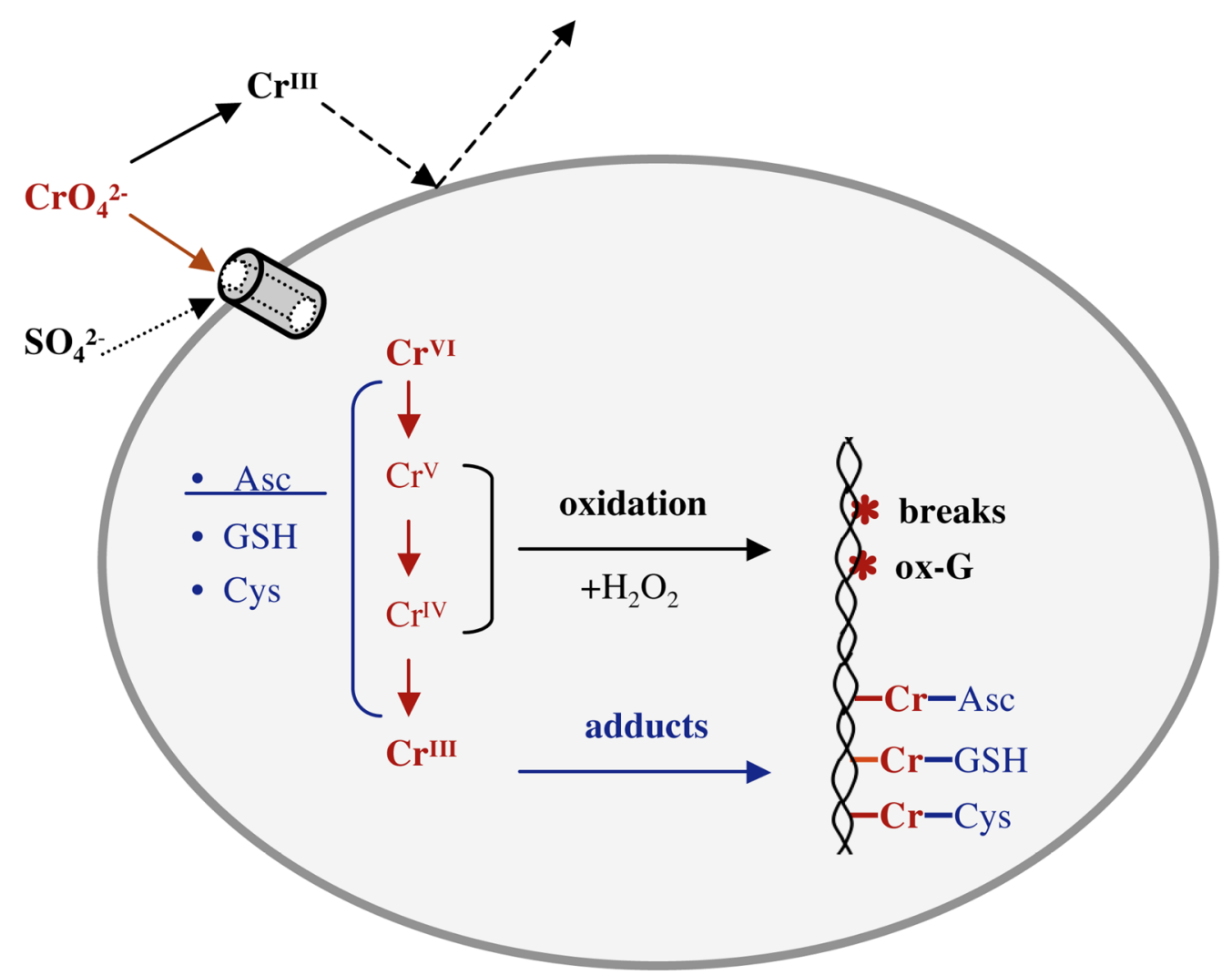

Figure I.5 Major steps in uptake, metabolism and formation of DNA damage by Cr (VI) (Salnikow and Zhitkovich, 2008)

NADPH oxidase activity and mitochondrial damage, which can ultimately lead to an increase of $\mathrm{H}_{2} \mathrm{O}_{2}$ level (Salnikow and Zhitkovich, 2008). In addition, it can result in the single-strand DNA (ssDNA) breaks in cells of animal tissues (Gao et al., 1992; Hodges et al., 2001; Sugiyama et al., 1986; Ueno et al., 2001). The generation of ssDNA breaks has been shown to be caused by oxidizing free radicals generated during the reactions between $\mathrm{Cr}(\mathrm{V})$ and $\mathrm{H}_{2} \mathrm{O}_{2}$ (Messer et al., 2006).

Since $\mathrm{Cr}(\mathrm{VI})$ can lead to an increase of $\mathrm{H}_{2} \mathrm{O}_{2}$ level and oxidative stress in cells, which can further cause additional severe biological consequences. Chromium VI can 
damage a variety of cellular components including proteins, lipids, DNAs and RNAs. Cr (VI)-induced oxidative DNA damage plays critical roles in causing genome instability and carcinogenesis (Kawanishi et al., 2002; Kawanishi et al., 2001). Earlier studies showed that chromate exposure can induce both ssDNA breaks and abasic sites (Casadevall and Kortenkamp, 1994, 1995). Although there is no direct evidence showing that chromate exposure can induce 8-oxoG, the indicator of oxidative damage on DNA, Slade et al. have reported that ascorbate-mediated $\mathrm{Cr}$ (VI) reduction yields significant amount of spirominodihydantoin, which is a guanine oxidation product (Slade et al., 2005) indicating that chromate can also attack guanine in DNA.

Taken together, both bromate and chromate exposure can cause severe oxidative DNA damage in the genome by attacking guanines in the DNA, in particular the guanines located at $\mathrm{G}$ rich regions such as $\mathrm{CpG}$ islands in the promoter region of genes as well as the regions containing trinucleotide repeats, which are the hotspots of DNA damage induced by bromate and chromate. If the mutations remain in the genome, they can further modulate genome and epigenome stability. Thus, understanding how bromate and chromate exposure can alter modulate genome and epigenome stability is important for us to further explore the etiology, prevention and diagnosis of environmentally-induced cancer. 


\section{B. BASE EXCISION REPAIR IS THE MAJOR PATHWAY TO REPAIR OXIDATIVE DNA DAMAGE AND PLAYS A CRITICAL ROLE IN ACTIVE DEMETHYLATION IN CpG DINUCLEOTIDE CLUSTERS.}

As summarized in the previous section, the unrepaired DNA damage can cause DNA base lesions, strand breaks, distortion of the double helix structure of DNA, ultimately mutations which can alter the expression of tumor suppressors or oncogenes promoting cell proliferation which will result in cancer development. In mammalian cells, different types of DNA damage are repaired thorough different DNA repair pathways as summarized in Figure I.1. The pathway that removes oxidative DNA damage including DNA base lesions and single-strand DNA breaks, which are the most common form of DNA damage, is the DNA base excision repair (BER) pathway (Neeley and Essigmann, 2006). The BER pathway was discovered more than thirty years ago, when Thomas Lindahl searched for enzymes that repair an uracil, which is the product of cytosine deamination. Later the enzyme that removes the lesion, uracil DNA glycosylase was discovered (Friedberg and Lindahl, 2004). The enzyme recognizes and removes the uracil which initiates the first step of the BER pathway (Barnes and Lindahl, 2004; David and Williams, 1998; Fromme and Verdine, 2004). There are 2 subpathways of BER that have been discovered, the short-patch BER (SP-BER, also known as single-nucleotide BER, (SN-BER) and long-patch BER (LP-BER) (Figure I-6). The difference between the two subpathways falls into the number of the nucleotides synthesized and replaced during the repair, as well as the repair enzymes and cofactors that participate in the repair process (Biade et al., 1998b; Fortini et al., 1998; Frosina et al., 1996; Klungland and Lindahl, 1997; Liu and Wilson, 2012). The enzymes that initiate the BER pathway are called DNA 
glycosylases (Fromme and Verdine, 2004). These enzymes catalyze the cleavage of the glycosidic bond that link a damaged base with a deoxyribose, thereby removing the base lesion from the DNA backbone (Fromme and Verdine, 2004). In human cells, different DNA glycosylases exhibit their specific activity to different types of base lesions (Brooks et al., 2013; Fromme et al., 2004; Sampath et al., 2012; Wallace, 2014). Uracil DNA glycosylase (UDG) that specifically removes an uracil. 8-oxoG DNA glycosylase 1

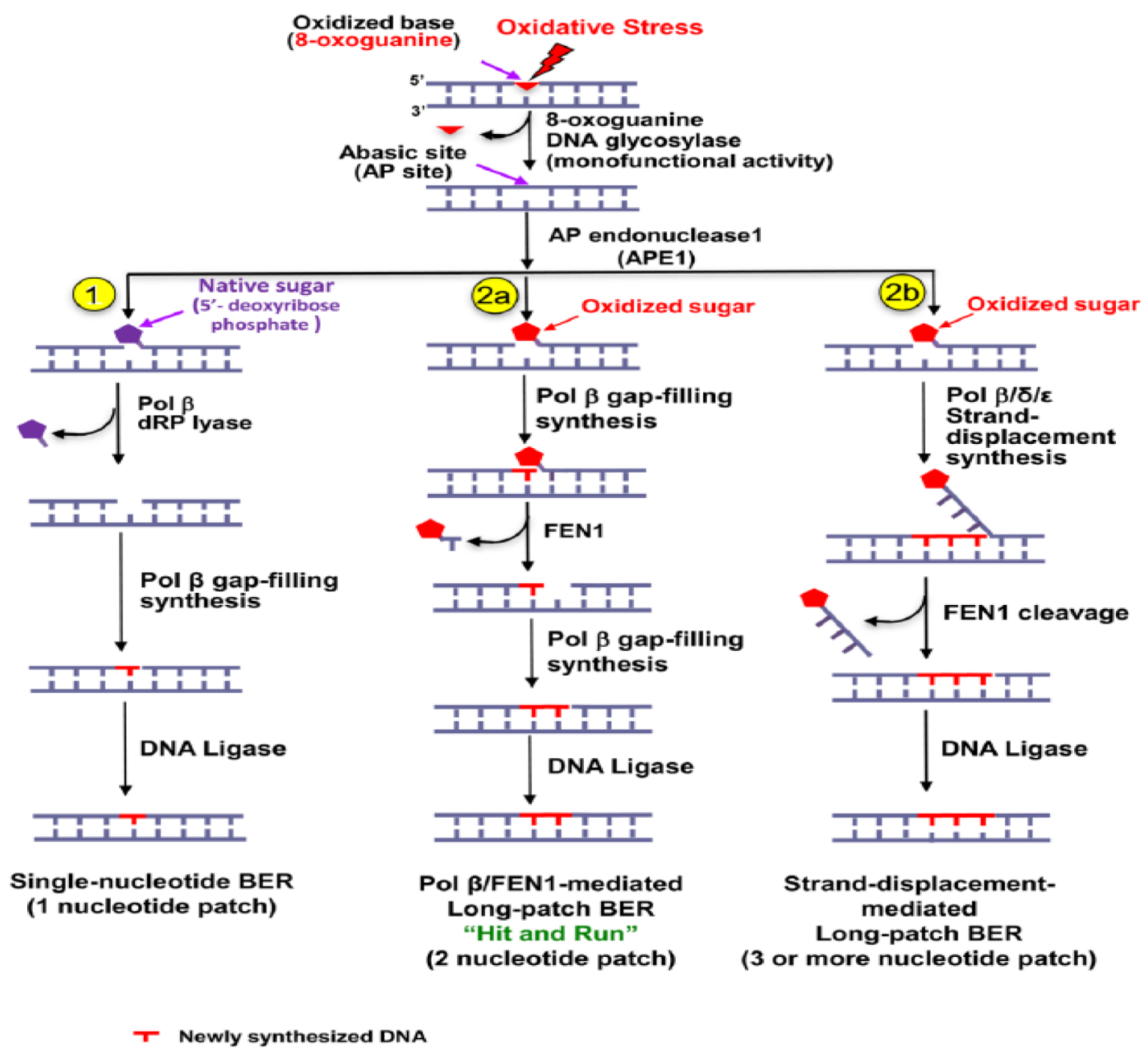

Figure I.6 Pathways of DNA Base Excision Repair (Liu and Wilson, 2012) 
(OGG1) specifically removes an 8-oxoG, and 3-alkyladenine DNA glycosylase (AAG) preferentially
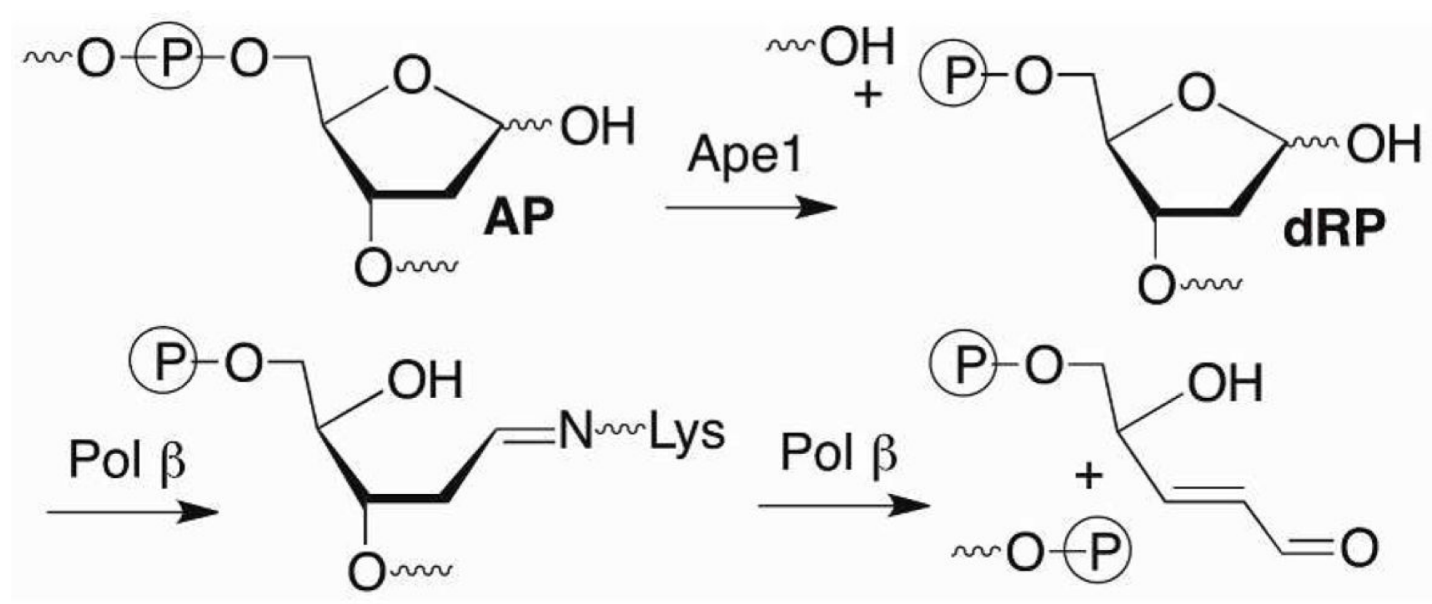

Figure I.7 The dRP lyase mechanism of pol $\boldsymbol{\beta}$ (Stevens et al., 2013)

remove an alkylated base such as 3-methyl-A. Removal of a base lesion will leave an abasic site (AP site), which is then recognized by AP endonuclease 1 (APE1). Subsequently, APE1 incises the AP site at the 5'-side of the site resulting in the formation of a onenucleotide gap with an 3'-hydroxyl in the upstream, and a 5'-dRP in the downstream (Liu and Wilson, 2012; Masuda et al., 1998; Wilson et al., 1995). Then the oxidation of the sugar on the 5'-dRP group determines which BER subpathway the lesion follows. If the 5'-dRP group is unmodified and remains as a native sugar, pol $\beta$ dRP lyase directly removes the group with its dRP lyase activity (Stevens et al., 2013) through $\beta$-elimination (Figure I.7) that involves the formation of a Schiff base and formation of a temporary pol $\beta$-DNA crosslink intermediate. The pol $\beta$ polymerase activity then synthesizes one nucleotide to fill in the gap generating a nick, which is then sealed by DNA Ligase I (LIG 
I) or DNA Ligase III (LIG III)-X-ray cross-complementing group 1 (XRCC1) complex. In above subpathway, the repair is accomplished with replacement of one nucleotide, i.e. SNBER or SP-BER.

However, if the 5'-dRP sugar is further oxidized by oxidative stress, pol $\beta \mathrm{dRP}$ lyase activity cannot remove the sugar. The repair of the lesion must be subject to LP-BER where pol $\beta$ fills the gap by synthesizing two or two more nucleotides. A 5'-sugar phosphate is then recognized and removed by flap endonuclease 1 (FEN1) (Figure I.6, subpathway 2a and 2b) (Frosina et al., 1996; Liu and Wilson, 2012). There are two subpathways for LP-BER, the "Hit and Run" subpathway and the "strand displacement synthesis" subpathway that fulfill the process of base lesion repair (Figure I.6). In the "Hit and Run" subpathway, APE1 incises an abasic site leaving a $1 \mathrm{nt}$ gap with a 5' modified sugar residue. One nucleotide is inserted by pol $\beta$ to fill the gap creating a sugar flap attached to a downstream nucleotide. Subsequently, FEN1 captures the dRP flap by cleaving the downstream nucleotide that is attached to the modified sugar, leaving an additional $1 \mathrm{nt}$ gap. The pol $\beta$ capture fill the gap, leaving a nick which is then sealed by LIG I. In the demonstrated subpathway, only two nt are replaced (Liu and Wilson, 2012). In another subpathway of LP-BER, three or more nucleotides are synthesized and replaced via the "strand displacement synthesis" that can be mediated by either pol $\beta$ or replication DNA polymerases, DNA polymerase $\delta$ (pol $\delta$ ) or DNA polymerase $\varepsilon($ pol $\varepsilon$ ). In this subpathway, after APE1 incision of an abasic site, pol $\beta$ and/or pol $\delta$ or pol $\varepsilon$ perform strand displacement synthesis by inserting 3 or more nucleotides to elongate the upstream strand. As a result, the downstream strand is displaced, resulting a 5'-flap (Dianov et al., 1999; Klungland and Lindahl, 1997; Parikh et al., 1998; Podlutsky et al., 2001). Flap 
endonuclease 1 (FEN1) can then capture the flap and be loaded flap through threading through the free 5 '-end of the flap and track down to the base of the flap where it makes cleavage of the flap, thereby removing the modified sugar (Harrington and Lieber, 1994; Murante et al., 1995; Prasad et al., 2000). The above process results in a nick that is sealed by LIG I completing LP-BER (Liu and Wilson, 2012). The efficiency of the LP-BER subpathways is also modulated by the coordination among BER enzymes and cofactors.

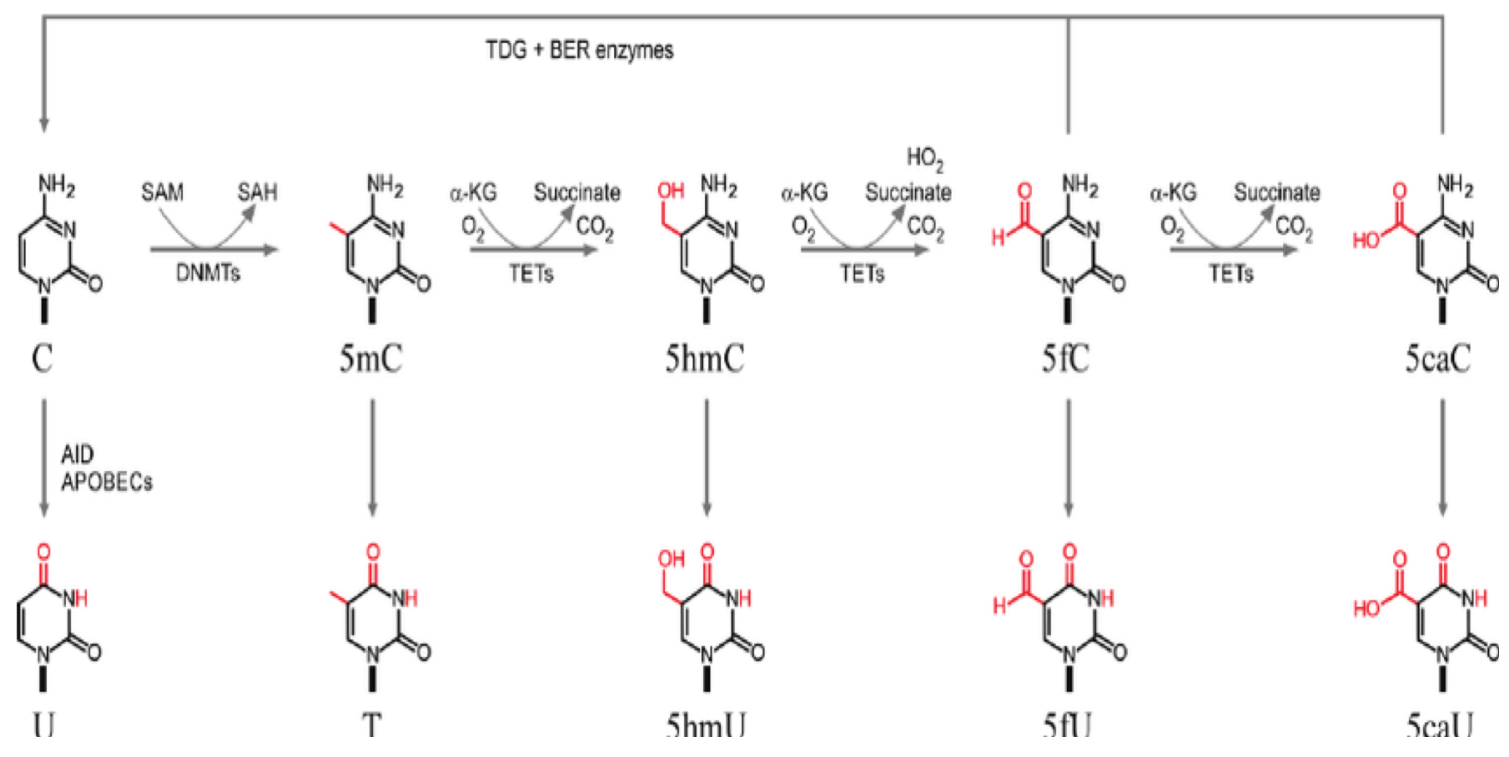

Figure I.8 Cytosine methylation and pathways of active DNA demethylation (Bacolla et al., 2014)

BER also plays essential role in mediating the active demethylation of 5'methylcytosines (5mCs). Active DNA demethylation regulates DNA methylation pattern in gene promoters and protein encoding regions, which plays critical roles in many biological processes including regulation of gene expression, retrovirus silencing, $\mathrm{X}$ chromosome inactivation among others (Ito and Kuraoka, 2015; Smith and Meissner, 2013). The pattern of DNA methylation, determined by the combination of methylation at the 5-position of a cytosine $(5 \mathrm{mC})$ and its demethylation at $\mathrm{CpG}$ clusters and islands in 
gene promoters, governs gene expression by regulating chromatin structures and accessibility of transcription factors to the promoter regions. If a $\mathrm{CpG}$ island in a promoter region is hypomethylated, i.e., a low level of $5 \mathrm{mCs}$, chromatin adopts an open configuration, allowing transcriptional factors to access the promoter regions to initiate gene transcription. If a $\mathrm{CpG}$ island is hypermethylated, i.e., a high level of $5 \mathrm{mCs}$, methylated Cs will attract methylC binding proteins (MBDs) to bind to the promoter region. The binding of MBDs to the promoter subsequently recruits histones that bear posttranslational modifications for a closed chromatin configuration, thereby preventing the binding of transcription factors to the DNA and inhibiting transcription initiation and silencing gene expression. Thus, hypermethylated CpG islands can serve as a "switch off" mark of a specific gene. By participating in the demethylation of $5 \mathrm{mCs}$ in the $\mathrm{CpG}$ islands, BER can modulate gene expression in embryonic and somatic cells (Bellacosa and Drohat, 2015; Drohat and Coey, 2016b; Fleming et al., 2017; Furlan et al., 2017; Li et al., 2013; Seisenberger et al., 2013).

The first step of the BER-mediated active demethylation is the oxidation of 5'methylcytosine that is catalyzed by the Ten-Elven Translocation (TET) oxidases during which $5 \mathrm{mCs}$ are converted to 5'-hydroxycytosines (5hmCs) (Figure I-8). Subsequently, an enzyme, AID/apolipoprotein B editing complex (APOBEC) protein, converts a $5 \mathrm{hmC}$ to a 5-hydroxymethyluracil (5hmU), which is recognized by the DNA glycosylase, singlestrand-selective monofunctional uracil-DNA glycosylase I (SMUG1). Furthermore, a 5mC can also be spontaneously deaminated into thymine, which can be recognized and removed by thymine DNA glycosylase (TDG). The oxygenases, TET 1, 2 and 3 enzymes can oxidize 5hmC into 5-formylcytosine (5fC) and 5-carboxylcytosine (5caC) (Figure I.8). Figure I-9 
illustrates the mechanisms by which TETs convert $5 \mathrm{mC}$ to $5 \mathrm{hmC}, 5 \mathrm{fC}$, and $5 \mathrm{caC}$ (Bochtler et al., 2017). The $5 \mathrm{fC}$ and $5 \mathrm{caC}$ base lesions that base pair with a guanine can also be recognized and removed by TDG (Bellacosa and Drohat, 2015; Drohat and Coey, 2016a; Gong and Zhu, 2011; Ito and Kuraoka, 2015; Mahfoudhi et al., 2016; Maiti and Drohat, 2011; Niehrs and Schafer, 2012; Wallace, 2014). Thus, the active DNA demethylation is mediated by enzymatic conversion of $5 \mathrm{mCs}$ into a series of modified cytosines that are recognized as DNA base lesions by DNA glycosylases. Removal of the DNA base lesions initiate the BER pathway that efficiently repairs the lesions generated from $5 \mathrm{mCs}$. Through $\mathrm{BER}$, a $5 \mathrm{mC}$ is replaced by an unmodified cytosine, thus resulting in the completion of active DNA demethylation (Wallace, 2014) that modulates gene expression.

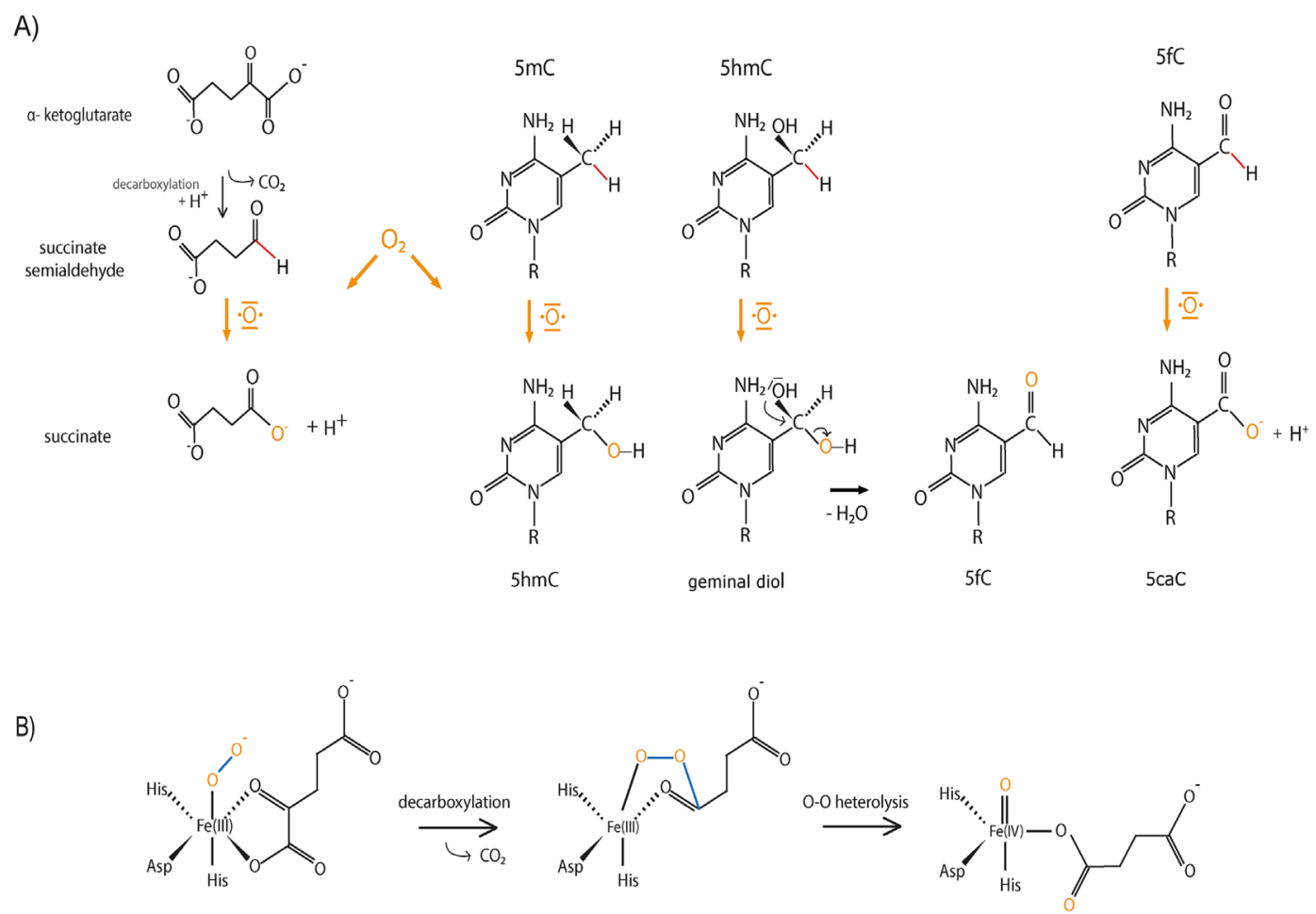

Figure I.9 TET-catalyzed oxidation reactions. A) Incorporation of an oxygen molecule into $5 \mathrm{mC}, 5 \mathrm{hmC}$, and $5 \mathrm{fC}$. B) Activation of molecular oxygen and formation of the $\mathrm{Fe}(\mathrm{IV})=\mathrm{O}$ intermediate (Bochtler et al., 2017). 


\section{DNA DAMAGE DISRUPTS DNA METHYLATION PATTERN BY MODULATING ACTIVITIES OF DNA METHYLTRANSFERASES (DNMTS).}

During DNA replication, mammalian cells, both genetic and epigenetic information including DNA methylation, chromatin structure and histone modifications, must be replicated (Mortusewicz et al., 2005). Methylation of a cytosine in the $\mathrm{CpG}$ islands is catalyzed by DNA methyltransferases (DNMTs), which transfer a methyl group from the methyl donor, S-adenosyl-L-methionine (SAM) to the 5-position of the cytosine in DNA (Robertson, 2005). The DNMTs family has four members including DNA methyltransferase 1 (DNMT1), DNMT3a, DNMT3b, and DNMT3L. Since DNMT3L does not exhibit any enzymatic activity in cells (Kareta et al., 2006), the mammalian DNA methylation is carried out by three DNA methyltransferases, DNMT1, DNMT3a, and DNMT3b.The compounds DNMT3a and $3 \mathrm{~b}$ can methylate a cytosine on $\mathrm{CpG}$ islands and are called de novo DNMTs. However, DNMT1 recognizes a hemi-methylated DNA strand as the substrate. Thus, it methylates cytosines at the newly synthesized DNA strand by recognizing a $5 \mathrm{mC}$ in the parental strand after DNA replication. As a result, DNMT1 sustains an established DNA methylation pattern (Chuang et al., 1997; Leonhardt et al., 1992). Thus, DNMT1 is called a maintenance DNA methyltransferase. Several studies also have shown that there is functional overlap between the de novo and maintenance methyltransferases (Egger et al., 2006; Riggs and Xiong, 2004), This indicates that DNMT1 may play more complicated roles in modulating methylation pattern than thought before. Previous studies showed that knockout of DNMT1 or DNMT3b resulted in

embryonic lethality in mice, indicating the critical role of DNA methylation during embryonic development. Previous studies have also found that the mutations of DNMTs 
caused genetic disorders (Li et al., 1992; Okano et al., 1999). Furthermore, disruption of a normal DNA methylation pattern such as the hypermethylation in the promoters of tumor suppressor genes and the global hypomethylation, is commonly detected in cancer cells (Jones and Baylin, 2002) indicating its association with cancer development. Because CpGs are composed of Cs and Gs that can be readily deaminated enzymatically and spontaneously as well as oxidized by endogenous and exogenous oxidative DNA damage, they can readily form hotspots of DNA base damage that can be repaired by BER. Recent studies have shown that DNMTs can cooperate with each other to efficiently methylate repetitive elements in the genome to prevent the expression of these elements in cells (Subramaniam et al., 2014). Moreover, earlier studies also show that DNMT1 helps restore the epigenetic information during DNA repair by interacting with a DNA repair cofactor, proliferating cell nuclear antigen (PCNA) (Mortusewicz et al., 2005). By interacting with PCNA through its PCNA binding domain, DNMT1 can be recruited to double strand DNA breaks (DSBs) (Chuang et al., 1997; Leonhardt et al., 1992; Mortusewicz et al., 2005). The recruitment of DNMT1 to DSBs is also mediated through the interaction between DNMT1 and Rad 9, which is a component of the Rad 9-Hus 1-Rad 1 sliding clamp (Ha et al., 2011). Furthermore, during DSB break repair mediated by homologous recombination (HR), DNMT1 can interact with the growth arrest and DNA-damage-inducible protein $\alpha$ (GADD45 $\alpha$ ) protein to precisely select the DNA strand that needs to be methylated (Lee et al., 2012). This suggests that DNMTs can modulate DNA repair by coordinating with DNA repair enzymes and cofactors. Since BER is the pathway to repair the small DNA lesions such as 8-oxoG as well as the modified DNA bases generated by active DNA demethylation, i.e., $5 \mathrm{hmC}, 5 \mathrm{fC}$, and $5 \mathrm{caC}$, it is possible that DNMT1 may also interact with 
BER enzymes such as pol $\beta$ during BER and/or active demethylation process. In this scenario, BER and DNMTs may crosstalk with each other on the sites with modified or damaged DNA bases to ensure the efficiency of damage repair as well as maintenance of integrity of DNA methylation and demethylation.

\section{BREAST CANCER 1 (BRCA1) PLAYS A CRITICAL ROLE IN PREVENTING THE DEVELOPMENT OF BREAST CANCER.}

Breast cancer is one of the most common invasive cancers in women and the leading cause of cancer death in women under 40 years old (Alabdulkareem et al., 2017). It has been found that development of breast cancer is associated with the breast cancer 1 gene (BRCA1), which is located at the $17^{\text {th }}$ chromosome in human genome (Figure I-10). The BRCA1 protein is a 1863 amino acid protein and functions as a tumor suppressor (Henderson, 2012). The problem is involved in DNA repair regulation, genome maintenance, cell survival, and apoptosis (Clark et al., 2012; Henderson, 2012; Long and Walter, 2012) and directly participates in repair of DSBs caused by endogenous and exogenous DNA damaging agents by interacting with other DNA repair proteins (Cousineau et al., 2005; Hu et al., 1999; Huen et al., 2010; Kote-Jarai and Eeles, 1999; Liu

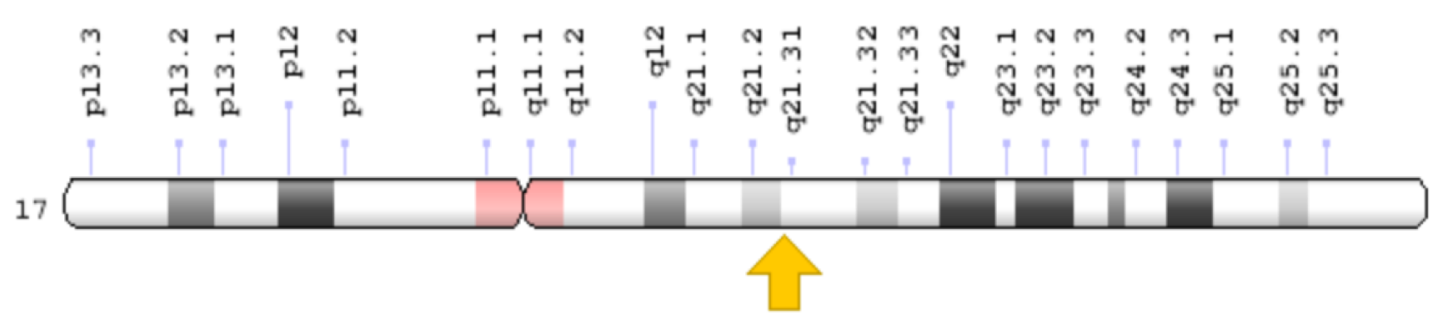

Figure I.10 BRCA1 gene is located on the $17^{\text {th }}$ chromosome in human genome (NIH U.S. National Library of Medicine BRCA1). 
et al., 2007a; Moynahan et al., 1999). For example, BRCA1 can interact with BRCA2 to promote efficient and precise repair of DSBs resulting in maintenance of genomic stability. During DSB repair, BRCA1 is recruited to DSB sites. This is mediated by ubiquitination of histone $\mathrm{H} 2 \mathrm{~A}$ and $\mathrm{H} 2 \mathrm{~B}$. Initially DSBs induces the phosphorylation of the histone protein, H2AX on the DNA damage via ATM and ATR (Burma et al., 2001). Subsequently, an E3 ubiquitin ligase RNF8 and a phosphor-module binding mediator MDC1 are recruited to DSB sites (Huen et al., 2007; Kolas et al., 2007; Mailand et al., 2007; Stucki et al., 2005). RNF8 then interacts with the $\mathrm{E} 3$ ubiquitin conjugase UBC14 to ubiquitinate histone $\mathrm{H} 2 \mathrm{~A}$ and $\mathrm{H} 2 \mathrm{~B}$ at the lesions in the chromatin. This then recruits BRCA1 to the DNA damage sites (Wu et al., 2009; Zhao et al., 2007). BRCA1 then recruits RAD51 to DSB sites mediating DNA strand branch migration during DSB repair via HR (Scully et al., 1997). BRCA1 also interacts with other DNA repair machineries and recruits them to the DNA damage sites. These include RAP80, CCDC98/Abraxas, NBA1/MERIT40, BRCC36 and BRCC45 (Feng et al., 2009; Kim et al., 2007; Liu et al., 2007c; Shao et al., 2009; Sobhian et al., 2007; Wang et al., 2009; Wang et al., 2007). The formation of the complex between these proteins and BRCA1 is mediated by a series of sequential steps starting from ubiquitination of histones at the sites of DNA damage. This is recognized by RAP80 leading to the formation of a BRCA1-RAP80 protein complex. Moreover, BRCA1 is also involved in the activation of cell cycle check point induced by DSBs to initiate cell cycle arrest. This allows cells to have sufficient time to repair the DNA damage (Hartwell and Kastan, 1994), thereby preventing the duplication of damaged DNA and passage of the damaged DNA to daughter cells. BRCA1 is involved in the activation of all the cell checkpoints including G1/S, S-phase, and G2/M checkpoints. BRCA1 can act as a scaffold 
protein to facilitate ATM-mediated $\mathrm{p} 53$ phosphorylation resulting in activation of $\mathrm{p} 21$ that eventually induces G1/S arrest (Fabbro et al., 2004). BRCA1 can participate in S-phase checkpoint initiation along with ATM and Nbs1 (Xu et al., 2001). Its roles in inducing the G2/M checkpoint have been most intensively studied. DNA damage can activate PI3kinase-like kinase (PI3KK), ATM and ATR (Greenberg et al., 2006). This subsequently activates a series of protein kinases that regulate cell-cycle checkpoints. For example, PI3KK, ATM, and ATR phosphorylate Chk1 on serine 317 and serine 345 (Cousineau et al., 2005; Xu et al., 2001; Zhao et al., 2002). This further activates Wee1 (Greenberg et al., 2006; O'Connell et al., 2000), which can then inactivates Cdc25 that dephosphorylates Thr14 and Thr15 of Cdc2. The inactivation of Cdc25 sustains the phosphorylated Thr14 and Thr15 of Cdc2 (Perry and Kornbluth, 2007; Yarden et al., 2002), thereby activating G2/M checkpoint. In addition, BRCA1 can cooperate with the reactive attachment disorder 3-reactive attachment disorder $26(\operatorname{Rad} 3-\operatorname{Rad} 26)$ complex to cause the phosphorylation of Ser317 and Ser345 on Chk1 that activates G2/M checkpoint. BRCA1 is also involved in DNA damage-induced activation of ATM and ATR (Greenberg et al., 2006). Furthermore, BRCA1 can cooperate with BRCA1-C-terminus-domain containing proteins (BRCTs), checkpoint loading complex (CLC), and checkpoint sliding clamp (CSC) to activate G2/M checkpoint (O'Connell et al., 2000). Also it has been shown that the phosphorylation of BRCA1 at serine 1423 by ATM is implicated to be important for activation of IR-induced G2/M checkpoint (Xu et al., 2001). Thus, BRCA1 plays multifaceted roles in maintaining genome stability by facilitating DSB repair and regulating the check points cell cycle. 


\section{OVERVIEW}

Cellular DNA is frequently damaged by endogenous and exogenous DNA damaging agents. It has been estimated that more than 10,000 base lesions are generated per cell per day. These lesions are efficiently repaired by the BER pathway. However, a base lesion with a covalent bond formed between the $\mathrm{C} 5$ of a deoxyribose and $\mathrm{C} 8$ of a purine such as 5',8-cyclopurine-2'-deoxynucleoside (cdPu) can be only repaired by NER with a low efficiency readily accumulating in the genome. Accumulated cdPu lesions can effectively block replication DNA polymerases, and have to be bypassed by lesion bypass synthesis via repair DNA polymerases for cell survival. Yet, the mechanisms by which cdPu lesions are bypassed remain to be elucidated. On the other hand, DNA bases are chemically modified at $\mathrm{CpG}$ clusters and islands located at gene promoters and protein encoding regions, and this serves as an essential step to initiate and regulate cellular epigenetic features through DNA methylation and demethylation mediated by BER for regulation of gene expression. However, CpGs can also form hotspots of DNA base damage such as uracils and thymines from deamination of Cs and 5mCs as well as 8-oxoGs. Thus, cellular DNA methylation and demethylation frequently converges with DNA base damage and repair. It appears that BER evolves as a central component in mediating the interplay between epigenetics and DNA damage and repair as a part of genome and epigenome interaction which can be further modulated by environmental toxicants such as bromate and chromate that can oxidize DNA bases. The DNA damage induced by the environmental toxicants may further alter DNA methylation pattern on the promoter regions of tumor suppressor genes, leading to cancer development. In CHARPTER 1, employing pol $\beta$ wild-type (WT) and pol $\beta$ knockout (KD) mouse embryonic fibroblast 
(MEF) cell extracts, we provided the first evidence showing that pol $\beta$ plays a critical role in bypassing a $R^{\prime}$-cdA lesion efficiently. We further demonstrated that pol $\beta$ performed nucleotide misinsertion in bypassing a $R^{\prime}$-cdA indicating a pol $\beta$-mediated mutagenic effect during its lesion bypass synthesis. In CHARPTER 2, we further examined the effects of an abasic lesion next to a DNA demethylation intermediate, the $\mathrm{T} / \mathrm{G}$ mismatch in a $\mathrm{CpG}$ dinucleotide on the activity of major BER enzymes and the integrity of CpGs. We showed that pol $\beta$ extended a mismatched T generated by DNA demethylation resulting in a $\mathrm{C}$ to T mutation. However, we found that the mismatched $\mathrm{T}$ was efficiently removed by APE1 3'-5' exonuclease activity, which prevented pol $\beta$-mediated mutations during BER and active DNA demethylation. In CHARPTER 3, we further explored the effects of environmental toxicants, bromate and chromate on the DNA methylation pattern of the human tumor suppressor, BRCA1 gene. We showed that oxidative DNA damage induced by bromate and chromate altered DNA methylation pattern by inducing additional DNA methylation sites, loss of DNA bases and point mutations in the promoter region of BRCA1 gene and encoding region of BRCA1 protein. Moreover, we provided the first evidence showing that DNMT1 was recruited to the DNA damage sites through the interaction with pol $\beta$, thereby resulting in additional DNA methylation sites. 
CHAPTER 1

BYPASS OF A 5',8-CYCLOPURINE-2'DEOXYNUCLEOSIDE BY DNA POLYMERASE $\beta$ DURING REPLICATION AND BASE EXCISION REPAIR LEADS TO NUCLEOTIDE MISINSERTIONS AND DNA STRAND BREAKS

\begin{abstract}
The DNA lesion 5',8-cyclopurine-2'-deoxynucleosides including 5',8-cyclo-dA (cdA) and 5',8-cyclo-dG (cdG) are induced by hydroxyl radicals resulting from oxidative stress such as ionizing radiation. 5',8-cyclopurine-2'-deoxynucleoside lesions are repaired by nucleotide excision repair with low efficiency, thereby leading to their accumulation in the human genome and lesion bypass by DNA polymerases during DNA replication and base excision repair (BER). The present study, for the first time, we discovered that DNA polymerase $\beta$ ( $\operatorname{pol} \beta$ ) efficiently bypassed a 5' $R$-cdA, but inefficiently bypassed a 5' $S$-cdA during DNA replication and BER. We found that cell extracts from pol $\beta$ wild-type mouse embryonic fibroblasts exhibited significant DNA synthesis activity in bypassing a cdA lesion located in replication and BER intermediates. However, pol $\beta$ knock-out cell extracts exhibited little DNA synthesis to bypass the lesion which indicates that pol $\beta$ plays an important role in bypassing a cdA lesion during DNA replication and BER. Furthermore, we demonstrated that pol $\beta$ inserted both a correct and incorrect nucleotide to bypass a cdA at a low concentration. Nucleotide misinsertion was significantly stimulated by a high concentration of pol $\beta$, indicating a mutagenic effect induced by pol $\beta$ lesion bypass synthesis of a 5',8-cyclopurine-2'-deoxynucleoside. Moreover, we found that bypass of a 5'S-cdA by pol $\beta$ generated an intermediate that failed to be extended by pol $\beta$, resulting in accumulation of single-strand DNA breaks. Our study provides the first evidence that pol $\beta$ plays an important role in bypassing a 5, 8 -cyclo-dA during DNA replication and
\end{abstract}


repair, as well as new insight into mutagenic effects and genome instability resulting from pol $\beta$ bypassing of a cdA lesion.

Keywords: 5',8-cyclopurine-2'-deoxynucleoside, DNA polymerase $\beta$, Lesion bypass synthesis, Base excision repair, DNA replication, Mutagenesis

\section{INTRODUCTION}

Reactive oxygen species (ROS) induced by endogenous oxidative stress and environmental factors such as ionizing radiation can attack genomic DNA and result in various types of DNA damage such as oxidized DNA base lesions, strand breaks and DNAprotein cross-links in the human genome (Cadet et al., 1999; Dizdaroglu et al., 2001b). Because of its high reactivity with DNA bases, the hydroxyl radical $\left({ }^{\circ} \mathrm{OH}\right)$ leads to the formation of the majority of oxidized base lesions (Cadet et al., 2003). One type of oxidized DNA base lesion induced by ${ }^{\bullet} \mathrm{OH}$ is the 5',8-cyclopurine-2'-deoxynucleosides (cdPu), which includes 5',8-cyclo-2'-deoxyadenosine (cdA) and 5',8-cyclo-2'-deoxyguanosine (cdG). These lesions can exist in a $5^{\prime} R$ or $5^{\prime} S$ configuration. It has been found that both configurations of cdPu lesions including $5^{\prime} R$-cdA and $5 ' S$-cdA lesions can form naturally in the genomic DNA of mammals (Chatgilialoglu et al., 2011b; Jaruga and Dizdaroglu, 2008; Mitra et al., 2012; Randerath et al., 2001; Shaked et al., 2012; Tilstra et al., 2012; Zhang et al., 2013). Because cdPu lesions caused by the highly reactive free radical, ${ }^{\circ} \mathrm{OH}$, which can be generated in cells by both endogenous and exogenous sources such as ionizing radiation and carcinogens, cdPu lesions have been also proposed to be used as an oxidative DNA damage marker to evaluate the level of oxidative stress in mammals (Chatgilialoglu et al., 2011b; Jaruga and Dizdaroglu, 2008; Wang, 2008). Previous studies 
have shown that $180-320$ cdPus/cell can be produced in fetal and postnatal rat liver per day (Randerath et al., 2001). In addition, it has been estimated that cdA lesions occur at a frequency of one lesion $/ 10^{7}$ DNA bases (Dizdaroglu et al., 2001a). In vitro studies have shown that the ratio of the amount of $5^{\prime} R$-cdA to $5^{\prime} S$-cdA in DNA is about 3:1 (Chatgilialoglu et al., 2011b), suggesting a different efficiency in production of various forms of cyclopurine lesions. The cdPus contain an extra covalent bond that links the C5' of the 2'-deoxyribose and $\mathrm{C} 8$ of the purine in addition to the glycosidic bond. The extra covalent bond can alter DNA structure (Chatgilialoglu et al., 2011a), thereby stabilizing the glycosidic bond of the lesions (Das et al., 2012; Kamakura et al., 2012; Lindahl and Wood, 1999) and preventing the cleavage of the bond by a DNA glycosylase (Das et al., 2012; Kamakura et al., 2012; Lindahl and Wood, 1999). Because 5',8-cyclopurine-2'deoxynucleoside lesions can induce distortions to double-stranded DNA that can be recognized by nucleotide excision repair (NER) proteins, NER is the only repair pathway that can remove this type of DNA damage (Brooks et al., 2000; Kropachev et al., 2014; Kuraoka et al., 2000). However, the efficiency of removal of a $5^{\prime} R$ - and $5^{\prime} S$-cdA lesion by NER is two- to four-fold less than that for other bulky DNA adducts such as a cis-B $[\alpha] \mathrm{P}-$ $\mathrm{N}^{2}$-dG adduct (Kropachev et al., 2014). The low efficiency of repairing cdPus results in the accumulation of a high level of cdPus in the genome which is supported by previous studies showing that a significant amount of cdPu lesions were detected in genomic DNA of cells and tissues of mammals and other organisms (Chatgilialoglu et al., 2011b; Dizdaroglu et al., 2001a; Jaruga and Dizdaroglu, 2008). The cdPus can severely impair normal cellular functions such as DNA replication and transcription (Yuan et al., 2011). It has been shown that both $5^{\prime} R$-cdA and $5^{\prime} S$-cdA can directly block RNA polymerase II 
synthesis by inhibiting recruitment of RNA polymerase II to a promoter region (Brooks et al., 2000; Marietta et al., 2002). The cdPus can also completely block DNA synthesis of human replication polymerases such as pol $\delta$, causing replication fork stalling (Kuraoka et al., 2000). To resolve the stalled replication fork and restart DNA replication, Y family DNA translesion polymerases are recruited to perform translesion synthesis to bypass the base lesions (Friedberg et al., 2005). However, a template 5'S-cdA can also block lesion bypass synthesis by $\mathrm{Y}$ family polymerases such as pol $\eta$ and pol $\mathrm{l}$, resulting in accumulation of DNA strand breaks and apoptosis (Kuraoka et al., 2001; Pednekar et al., 2014; You et al., 2013). Furthermore, the lesion bypass of a cdA by Y family polymerases is highly mutagenic (You et al., 2013). Thus, unrepaired cdPus in the genome may lead to adverse biological effects, resulting in the development of human diseases and pathological conditions such as cancer, neurodegeneration, and aging (Brooks, 2008; Jaruga and Dizdaroglu, 2008; Kirkali et al., 2009; Wang et al., 2012; Wang et al., 2011).

DNA polymerase $\beta$ (pol $\beta$ ) is the smallest DNA polymerase identified in cells thus far (Beard and Wilson, 2014). It belongs to the X family of DNA polymerases (Braithwaite and Ito, 1993). Pol $\beta$ is composed of two subdomains, a $31 \mathrm{kDa}$ polymerase domain and an $8 \mathrm{kDa}$ deoxyribose phosphate (dRP) lyase domain (Beard and Wilson, 2006). Pol $\beta$ plays an essential role in filling gaps and removing a 5'-dRP group during BER (Beard and Wilson, 2006, 2014). Similar to the Y family translesion synthesis polymerases, pol $\beta$ can also perform translesion synthesis to bypass a DNA base lesion during replication and BER (Bassett et al., 2002a; Batra et al., 2006; Beard et al., 2009; Chary et al., 2012a; Efrati et al., 1997; Hashim et al., 1997b; Hoffmann et al., 1996b; Maga et al., 2009b; Servant et al., 2002b; Vaisman and Chaney, 2000b; Villani et al., 2011b). Previous studies have shown 
that pol $\beta$ can bypass small base lesions including 8 -oxoG and apurinic/apyrimidinic sites (AP sites), as well as bulky DNA lesions including a cisplatin adduct, propano-2'deoxyguanosine (PdG), benzo(a)pyrene expoxide (BaP), DNA adduct, BaP-dG adduct, pyrimidine(6-4)pyrimidone photoproduct (6,4-PP) and cyclobutane pyrimidine dimer (CPD) (Bassett et al., 2002a; Batra et al., 2006; Beard et al., 2009; Chary et al., 2012a; Efrati et al., 1997; Hashim et al., 1997b; Hoffmann et al., 1996b; Maga et al., 2009b; Servant et al., 2002b; Vaisman and Chaney, 2000b; Villani et al., 2011b). Because pol $\beta$ lacks 3'-5' exonuclease activity for proofreading, it can insert an incorrect nucleotide to bypass a DNA base lesion, leading to a high frequency of mutation (Bassett et al., 2002a; Batra et al., 2006; Beard et al., 2009; Beard and Wilson, 2014; Chary et al., 2012a; Efrati et al., 1997; Hashim et al., 1997b; Hoffmann et al., 1996b; Servant et al., 2002b; Vaisman and Chaney, 2000b). For example, during its bypass of an AP site, pol $\beta$ can insert an adenosine opposite to the lesion that may ultimately result in $\mathrm{C}$ to $\mathrm{T}$ transition if the abasic site is originally derived from an 8-oxoG (Beard et al., 2009). Pol $\beta$ bypass of bulky DNA adducts such as Pt-adducts can also induce mutations in the genome with a high frequency (Bassett et al., 2002a; Batra et al., 2006; Chary et al., 2012a; Hashim et al., 1997b; Hoffmann et al., 1996b; Servant et al., 2002b; Vaisman and Chaney, 2000b). Thus, pol $\beta$ lesion bypass DNA synthesis can lead to mutations that may further result in the development of human cancer.

Previous studies have shown that several Y family DNA translesion synthesis polymerases can bypass a cdPu lesion during DNA replication with moderate efficiency (You et al., 2013). It remains to be elucidated whether other DNA repair polymerases that can bypass DNA base lesions, such as pol $\beta$, may also bypass a cdPu during DNA 
replication and repair and whether the lesion bypass of a cdPu by these DNA polymerases
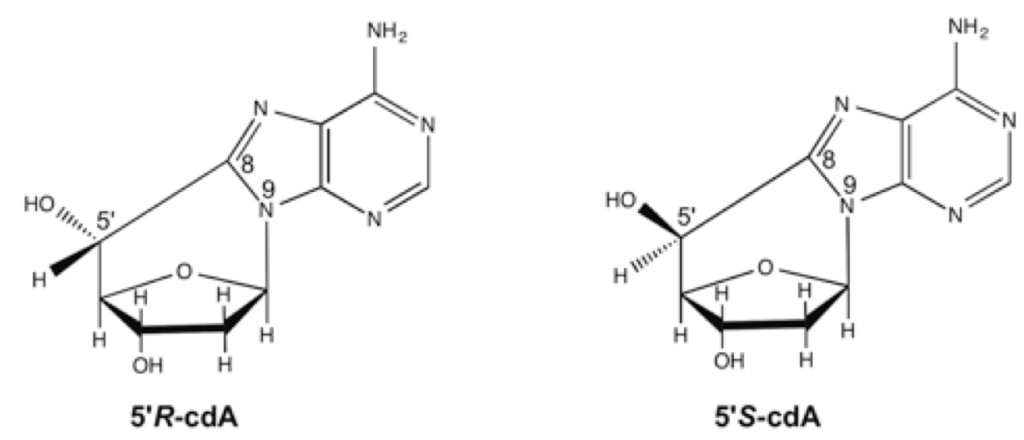

Figure 1.1 The structures of 5',8-cyclo-2'-deoxyadenosines.

may affect genome integrity and stability. We hypothesized that pol $\beta$ can bypass a $5^{\prime} R$ cdA and a 5'S-cdA during DNA replication and BER, and this may subsequently cause genome instability. Our hypothesis is supported by our recent study showing that pol $\beta$ can bypass both a $5^{\prime} R$-cdA and $5^{\prime} S$-cdA located in a CAG repeat tract by skipping over the lesions, thereby resulting in CTG repeat deletion (Xu et al., 2014). To test this hypothesis, we initially examined DNA synthesis for bypassing a cdA with pol $\beta$ deficient and proficient cell extracts. We then characterized pol $\beta$ DNA synthesis in bypassing a cdA during DNA replication and BER and determined nucleotide insertions during pol $\beta$ lesion bypass of a template cdA. For the first time, we found that pol $\beta$ efficiently bypassed a $5^{\prime} R-$ cdA during DNA replication and BER. However, the enzyme bypassed a 5' $S$-cdA with a low efficiency. Moreover, we further demonstrated that pol $\beta$ bypass of a cdA also led to 
nucleotide misinsertions and single-strand DNA breaks, indicating that pol $\beta$ lesion bypass of a cdA lesion during DNA replication and repair can induce mutations and strand breaks in the genome that ultimately results in genome instability.

\section{MATERIALS AND METHODS}

\subsection{Materials}

The DNA oligonucleotides containing a $5^{\prime} R$-cdA or $5^{\prime} S$-cdA were synthesized and purified by HPLC as described previously (Chatgilialoglu et al., 2014). The structures of $5^{\prime} R$-cdA and $5^{\prime} S$-cdA were illustrated in Figure 1.1. All other oligonucleotides were synthesized by Integrated DNA Technologies (IDT, Coralville, IA, USA). Deoxynucleoside 5'-triphosphates (dNTPs) were purchased from Fermentas (Glen Burnie, MD, USA). Radionucleotides, $\left[\gamma_{-}{ }^{32} \mathrm{P}\right]$ ATP $(6000 \mathrm{mCi} / \mathrm{mmol})$ and cordycepin 5 'triphosphate $3^{\prime}-\left[\alpha{ }^{32} \mathrm{P}\right](5000 \mathrm{mCi} / \mathrm{mmol})$ were purchased from Perkin Elmer Inc. (Boston, MA, USA). Micro Bio-Spin 6 chromatography columns were from Bio-Rad (Hercules, CA, USA). All other standard chemical reagents were from Sigma-Aldrich (St. Louis, MO, USA) and Thermo Fisher Scientific (Pittsburgh, PA, USA). Purified pol $\beta$, flap endonuclease 1 (FEN1) and DNA ligase I (LIG I) were generous gifts from Dr. Samuel H. Wilson at the National Institute of Environmental Health Sciences (NIEHS)/National Institutes of Health, Research Triangle Park, North Carolina.

\subsection{Oligonucleotide substrates}

Substrates containing a 31-nt template strand with a $5^{\prime} R-\operatorname{cdA}$ or $5^{\prime} S$-cdA located at

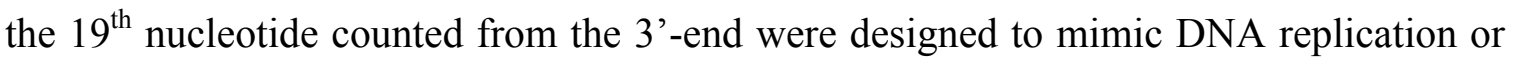
BER intermediates with a cdA lesion on the template strand. The upstream primer of the substrates is an 18-nt strand without or with a 3'-terminus dT that base paired with the 
template cdA. The downstream primer of the substrates is a 13 -nt strand with a 5'phosphate or 5'-phosphorylated tetrahydrofuran (THF) residue, an abasic site analog. Substrates that mimic the lesion bypass intermediates formed during DNA leading strand synthesis were constructed by annealing an18-nt upstream primer containing a 3'terminus $\mathrm{dT}$ with the cdA-containing template strand at a molar ratio of 1:2. Substrates representing the intermediates with a 1-nt gap opposite a template cdA formed during DNA lagging strand synthesis and BER were constructed by annealing the 18-nt upstream primer and a 13-nt downstream primer with a 5'-phosphate or 5'-THF residue with the template strand containing a $5^{\prime} R$-cdA or $5^{\prime} S$-cdA at a molar ratio of 1:1:2. Substrates were radiolabeled at the 5 '-end of the upstream primers or the 3 '-end of downstream primers for measuring the activities of different enzymes. The sequences of the oligonucleotide substrates are listed in Table 1.1.

Table 1.1 Sequences of the oligonucleotide substrates

\begin{tabular}{lcl}
\hline $\begin{array}{l}\text { Downstream } \\
\text { strand }\end{array}$ & nt & Seq. 5'-3' \\
\hline D1 & 13 & pGCGGATCCGGTGC \\
D2 & 13 & pFGCGGATCCGGTGC \\
\hline Template strand & & \\
\hline T1 & 31 & GCACCGGATCCGC $(5, \boldsymbol{R}) \boldsymbol{A C C C G G C A T C A G C T G C A G ~}$ \\
T2 & 31 & GCACCGGATCCGC $(5, \boldsymbol{S}) \boldsymbol{A C C C G G C A T C A G C T G C A G ~}$ \\
\hline Upstream strand & & \\
\hline U1 & 17 & CTGCAGCTGATGCCGGG \\
U2 & 18 & CTGCAGCTGATGCCGGGT \\
\hline F: tetrahydrofuran, THF &
\end{tabular}




\subsection{Translesion DNA synthesis in bypassing a cdA in mouse embryonic fibroblast (MEF) cell extracts}

The MEF cell extracts were prepared according to the procedure described previously (Biade et al., 1998a). Briefly, pol $\beta$ null $\left(\operatorname{pol} \beta^{-/-}\right.$) and wild-type pol $\beta$ (pol $\beta^{+/+}$) MEF cells were grown to near confluence. Cells were then harvested and lysed with rotation at $4{ }^{\circ} \mathrm{C}$ in lysis buffer. Cell lysates were then subject to centrifugation at 12,000 rpm for 30 min to obtain whole-cell extracts. The whole-cell extracts were subsequently dialyzed into BER reaction buffer containing $50 \mathrm{mM}$ Tris- $\mathrm{HCl}, \mathrm{pH}$ 7.5, $50 \mathrm{mM} \mathrm{KCl,} 0.1$ $\mathrm{mM}$ EDTA, $0.1 \mathrm{mg} / \mathrm{ml}$ bovine serum albumin and $0.01 \%$ Nonidet P-40. Levels of pol $\beta$ protein in different cell extracts were determined by immunoblotting using rabbit antimouse pol $\beta$ antibody (a generous gift from Dr. Samuel H. Wilson at National Institute of Environmental Health Sciences/National Institutes of Health) (Data not shown). The DNA synthesis activity was measured by incubating $60 \mu \mathrm{g}$ of cell extracts with open template substrates with an upstream primer, that contained a $5^{\prime} R$-cdA or $5^{\prime} S$-cdA on the template strand, or with gapped substrates with a 1-nt gap opposite a template $5^{\prime} R$-cdA or $5^{\prime} S$-cdA with a 5'-phosphate or 5'-phosphorylated THF residue. Reaction mixtures were assembled on ice and incubated at $37^{\circ} \mathrm{C}$ for $30 \mathrm{~min}$. Reactions were terminated by addition of $15 \mu \mathrm{l}$ of stopping buffer containing 95\% formamide and $10 \mathrm{mM}$ EDTA and subsequent incubation at $95{ }^{\circ} \mathrm{C}$ for $5 \mathrm{~min}$. Substrates and DNA synthesis products were separated by $15 \%$ or $18 \%$ urea-denaturing PAGE and detected by Pharos FX Plus PhosphorImager from Bio-Rad (Hercules, CA, USA). 


\subsection{In vitro BER reconstituted with purified enzymes}

In vitro $\mathrm{BER}$ of an abasic lesion that is opposite acdA was performed by incubating purified pol $\beta$, FEN1, LIG I with the substrates containing a 1-nt gap opposite a 5 ' $R$-cdA or a $5 ' S$-cdA on the template with a downstream 5'-THF residue. The $10-\mu 1$ reactions were reconstituted with the indicated concentrations of BER enzymes and substrates in BER reaction buffer with $50 \mu \mathrm{M}$ dNTPs, $5 \mathrm{mM} \mathrm{MgCl}_{2}$ and $2 \mathrm{mM}$ ATP. Reaction mixtures were assembled on ice and incubated at $37^{\circ} \mathrm{C}$ for $30 \mathrm{~min}$. Reactions were terminated by addition of stopping buffer containing 95\% formamide and $10 \mathrm{mM}$ EDTA and incubation at $95{ }^{\circ} \mathrm{C}$ for 5 min. Substrates and products were separated by $15 \%$ or $18 \%$ urea-denaturing PAGE and detected by Pharos FX Plus PhosphorImager. All substrates were ${ }^{32} \mathrm{P}$-labeled at the 5'end of the upstream primer.

\subsection{Enzymatic activity assays}

Lesion bypass synthesis activity of pol $\beta$ was determined with the open template and gapped substrates with a $5^{\prime} R$-cdA or $5^{\prime} S$-cdA in the template. Pol $\beta$ lesion bypass DNA synthesis activity on the open template was determined with the substrates without or witha 3'-terminus dT that is opposite a template $5^{\prime} R$-cdA or 5 ' $S$-cdA. The lesion bypass DNA synthesis activity was determined at $37^{\circ} \mathrm{C}$ for $30 \mathrm{~min}$ in a $10-\mu l$ reaction mixture in BER reaction buffer with $50 \mu \mathrm{M}$ dNTPs and $5 \mathrm{mM} \mathrm{MgCl}_{2}$. Reaction mixtures containing various concentrations of pol $\beta$ and $25 \mathrm{nM}$ substrates and BER buffer with $5 \mathrm{mM} \mathrm{MgCl} 2$ and 50 $\mu \mathrm{M}$ dNTPs, were assembled on ice and incubated at $37^{\circ} \mathrm{C}$ for $30 \mathrm{~min}$. FEN1 cleavage activity was measured by incubating the substrates containing a 1-nt gap without or with a downstream 5'-phosphorylated THF with the enzyme in the absence or presence of pol $\beta$ in BER buffer with $5 \mathrm{mM} \mathrm{MgCl}$ and $50 \mu \mathrm{M}$ dNTP at $37^{\circ} \mathrm{C}$ for $30 \mathrm{~min}$. Reactions were 
terminated by addition of stopping buffer containing 95\% formamide and $10 \mathrm{mM}$ EDTA and incubation at $95^{\circ} \mathrm{C}$ for $5 \mathrm{~min}$. Substrates and products were separated by $15 \%$ or $18 \%$ urea-denaturing PAGE and detected by a Pharos FX Plus PhosphorImager.

\section{RESULTS}

\subsection{Pol $\beta$ plays an important role in bypassing a cdA lesion}

Pol $\beta$ is a key enzyme that plays an essential role in mediating efficient BER in mammalian cells by removing a $5^{\prime}$-dRP group as well as filling in a single- or multinucleotide gap (Liu and Wilson, 2012). It also plays a critical role in lesion bypass synthesis during DNA leading and lagging strand synthesis (Maga et al., 2009b; Villani et al., 2011b) to facilitate Okazaki fragment maturation (Maga et al., 2009b). Therefore, pol $\beta$ may also play an important role in bypassing a cdA lesion during DNA replication and BER. To determine whether pol $\beta$ can play a role in bypassing a cdA, we initially determined DNA synthesis in bypassing a $5^{\prime} R$-cdA and $5^{\prime} S$-cdA in cell extracts with pol $\beta$ deficiency (pol $\beta^{-}$ ${ }^{-}$) and pol $\beta$ proficiency $\left(\mathrm{pol} \beta^{+/+}\right.$) with the open template substrates containing a $5 ' R$-cdA or 5 ' - -cdA on the template strand (Figure 1.2A) as well as the $1 \mathrm{nt}$-gapped substrates containing a template $5^{\prime} R$ - or $5^{\prime} S$-cdA with a $5^{\prime}$-phosphate (Figure 1.2B) and 5'phosphorylated THF residue (Figure 1.2C). The results showed that a significant amount of lesion bypass products were generated with the substrates containing a template $5^{\prime} R$ cdA in pol $\beta^{+/+}$cell extracts (lane 3 of Figure 1.2A, 2B and 2C). However, pol $\beta^{-/-}$cell extracts exhibited very weak lesion bypass synthesis with the substrates (lane 2 of Figure 1.2A, 2B, and 2C). On the other hand, pol $\beta^{+/+}$cell extracts performed weak DNA synthesis on the substrates containing a template 5'S-cdA (lane 6 of Figure 1.2A, 2B and 2C). 
However, little lesion bypass synthesis products was generated by pol $\beta^{-/-}$cell extract with

A

B
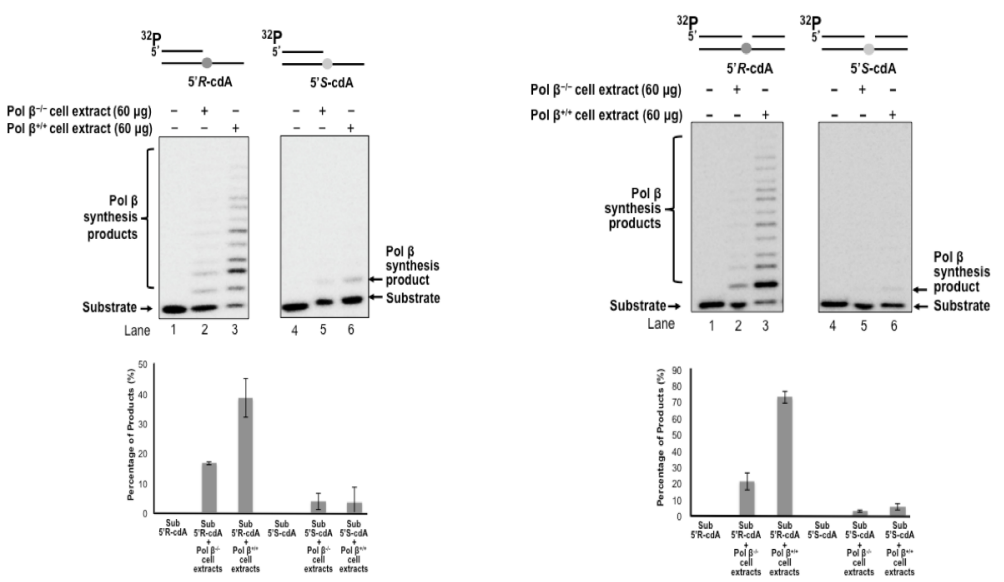

C
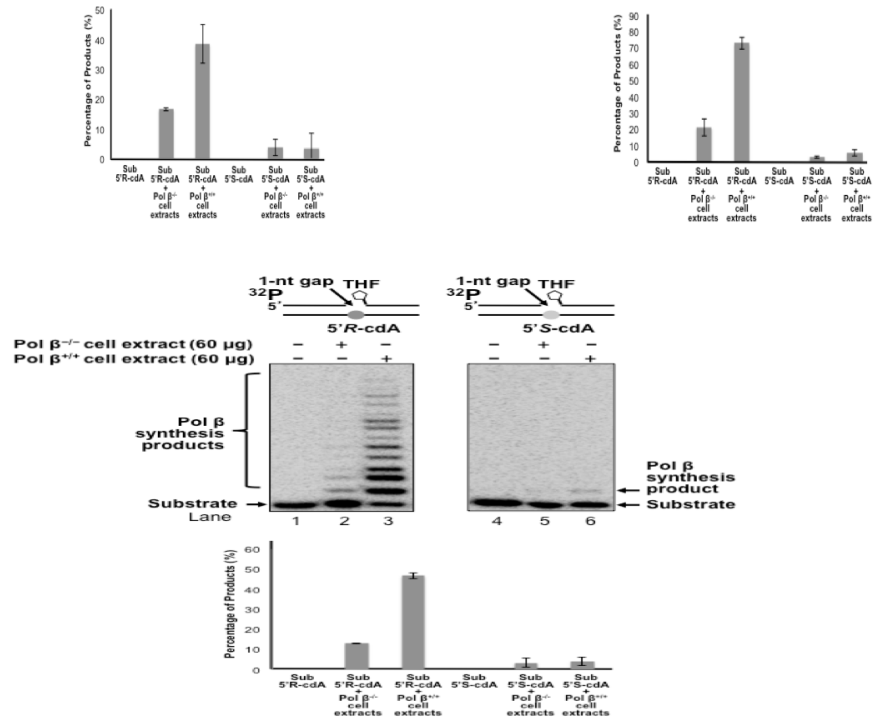

Figure 1.2 Pol $\beta$ plays an important role in bypassing a cdA lesion during DNA replication and BER. To test whether pol $\beta$ is involved in bypassing a cdA lesion during DNA replication (the leading strand synthesis and Okazaki fragment maturation) and BER, DNA synthesis was examined by incubating cell extracts of pol $\beta^{-/-}$and pol $\beta^{+/+}$MEFs with open template substrates that contained a template $5^{\prime} R$-cdA or $5^{\prime} S$-cdA (A) and 1-nt gapped substrates containing a template $5^{\prime} R$-cdA or $5^{\prime} S$-cdA (B) under the conditions described in Section 2. (C) Pol $\beta$ DNA synthesis in bypassing a template cdA lesion during BER was measured by incubating substrates that contained a 1 -nt gap opposite a template 5 ' $S$-cdA or 5 ' $R$-cdA and the downstream primer containing a 5'-phosphorylated THF residue. In all panels, Lanes 1 and 4 correspond to substrates only. Lanes 2 and 5 correspond to reaction mixtures with $60 \mu \mathrm{g}$ pol $\beta^{-/-}$MEFs extracts. Lanes 3 and 6 correspond to reaction mixtures with $60 \mu \mathrm{g}$ pol $\beta^{+/+}$MEFs extracts. The bottom panels illustrate the quantitative analysis of pol $\beta$ DNA synthesis in bypassing a cdA lesion of the substrates. Substrates were ${ }^{32}$ P-labeled at the 5'-end of the upstream primer as indicated. Substrates are illustrated schematically above the gel.

the substrates (lane 5 of Figure 1.2A, 2B and 2C). The results showed that in the presence 
of pol $\beta$, the amount of lesion bypass products was increased by about 2 to 5 -fold (the bottom panel of Figure 1.2A, 2B and $2 \mathrm{C}$ ) which indicated that pol $\beta$ played an important role in bypassing a cdA lesion during DNA replication and BER.

\subsection{Pol $\beta$ bypasses a 5' $R$-cdA efficiently and stalls at a 5' $S$-cdA}

Previous studies have shown that some $\mathrm{Y}$ family translesion synthesis polymerases such as pol $\eta$ can bypass a $5^{\prime} R$-cdA and 5' $S$-cdA with different efficiency (Kuraoka et al., 2000; Kuraoka et al., 2001). To further determine if pol $\beta$ can bypass a 5' $R$-cdA and 5' $S$ cdA during DNA replication and BER, we examined pol $\beta$ DNA synthesis in bypassing cdA lesions by incubating increasing concentrations of purified pol $\beta$ with open template substrates, as well as $1 \mathrm{nt}$-gapped substrates containing a template cdA. The results showed that with the open template substrates, $10 \mathrm{nM}$ of pol $\beta$ efficiently bypassed a $5^{\prime} R$-cdA and performed its multi-nucleotide DNA synthesis (Figure 1.3A, lane 2). With increasing concentrations of pol $\beta$ ( $25 \mathrm{nM}$ and $50 \mathrm{nM})$, the amount of pol $\beta$ DNA synthesis products from bypass of a $5^{\prime} R$-cdA was significantly increased (Figure 1.3A, lanes 3-4). However, pol $\beta$ exhibited weak DNA synthesis activity during its bypass of a 5'S-cdA (Figure 1.3A, lane 6). With increasing concentrations of pol $\beta$, the amount of its 1-nt insertion product was increased along with a slight increase in its multi-nucleotide DNA synthesis (Figure $1.3 \mathrm{~A}$, lanes 7-8). The results indicated that pol $\beta$ efficiently bypassed a $5^{\prime} R$-cdA, but stalled after it inserted a dT for bypassing a 5'S-cdA. The stalled pol $\beta$ DNA synthesis suggests that the base lesion conferred a strong inhibitory effect on polymerase lesion bypass synthesis during DNA leading strand synthesis. To further determine whether pol $\beta$ can

continue to perform its lesion bypass synthesis after it inserts one nucleotide that base pairs 
with a template cdA, we examined pol $\beta$ synthesis activity with the open template substrates containing a $3^{\prime}$-terminus dT that base paired with a template $5^{\prime} R$-cdA or $5^{\prime} S$ $\mathrm{cdA}$ (Figure 1.3B). These substrates mimic the intermediates resulting from pol $\beta$ 1-nt insertion that are generated during leading strand synthesis. We found that pol $\beta$ at 10-50 $\mathrm{nM}$ performed efficient DNA synthesis to extend a dT, which base paired with a $5^{\prime} R$-cdA (Figure 1.3B, lanes 2-4). However, the polymerase failed to extend a dT that base paired with a 5 ' $S$-cdA (Figure 1.3B, lanes 6-8), indicating that the 5 'S-cdA lesion completely inhibited pol $\beta$ DNA synthesis. Similar to its lesion bypass DNA synthesis with the open template substrates, pol $\beta$ exhibited efficient DNA synthesis with the 1-nt gapped substrates containing a $5^{\prime} R$-cdA without or with a $5^{\prime}$-phosphorylated THF residue that mimic a lagging strand maturation and BER intermediate, respectively (Figure 1.3C, lanes 2-4 and lanes 10-12). However, with the gapped substrates containing a 5 ' $S$-cdA, the enzyme only inefficiently inserted one nucleotide to base pair with the lesion (Figure 1.3C, lanes 6-8 and lanes 14-16). For the nicked substrates containing a 3'-terminus $\mathrm{T}$ that base paired with a $5^{\prime} R$-cdA without or with a $5^{\prime}$-THF residue, pol $\beta$ efficiently extended the dT (Figure 1.3D, lanes 2-4 and lanes 10-12), but failed to extend a 3'-terminus dT that paired with a 5'S-cdA (Figure 1.3D, lanes 6-8 and lanes 14-16). The results indicated that pol $\beta$ effectively bypassed a $5^{\prime} R$-cdA, but stalled at a $5^{\prime} S$-cdA after it inserted a dT that base paired with the lesion which further suggests that a $5^{\prime} S$-cdA on the template distorted the DNA double-helix structure. This subsequently disrupted pol $\beta$ binding to the substrates and its nucleotide insertion and further inhibited pol $\beta$ multi-nucleotide DNA synthesis during its lesion bypass. 

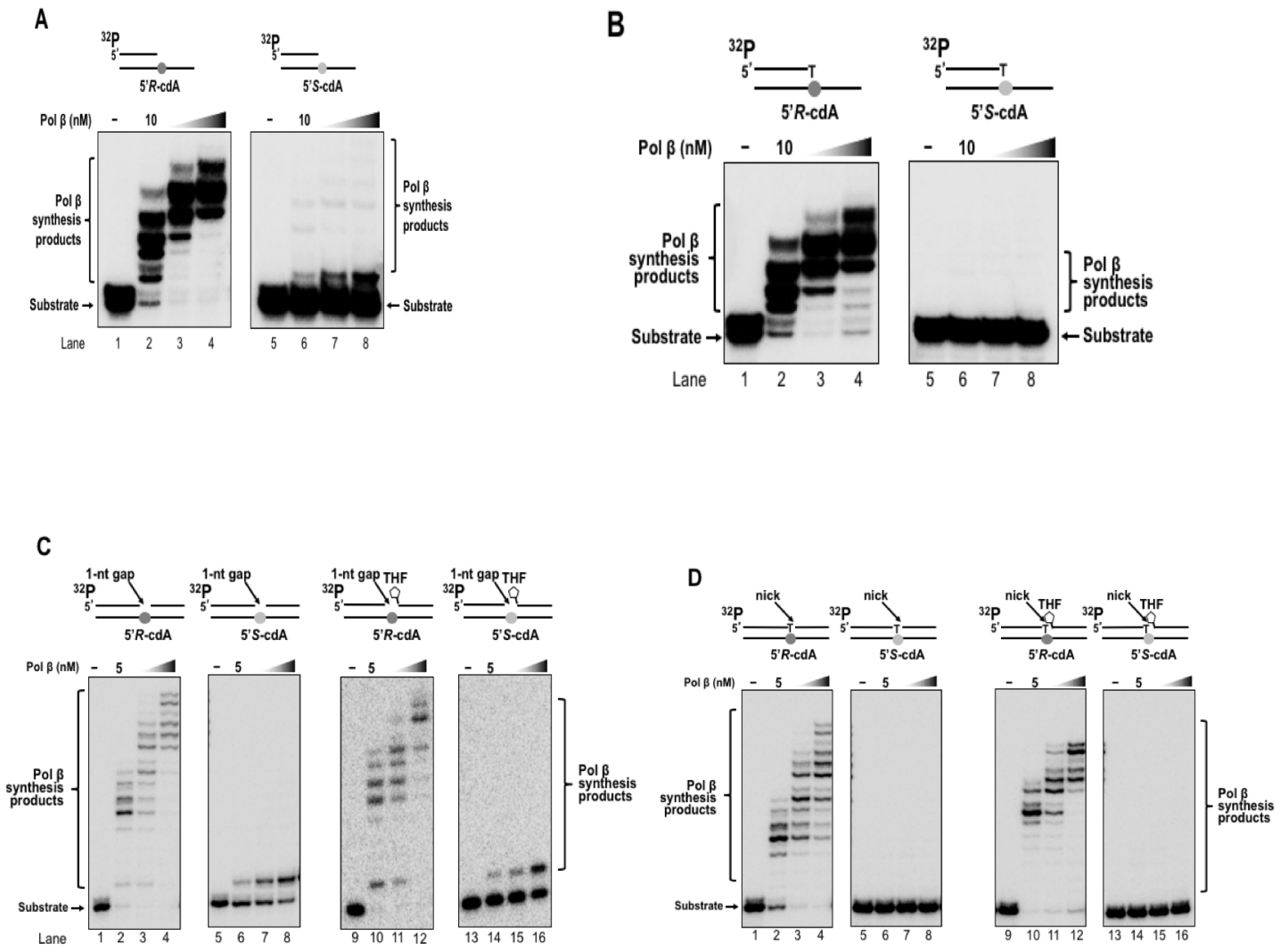

Figure 1.3 Pol $\beta$ DNA synthesis with a template cdA lesion during DNA replication and BER. (A) Pol $\beta$ bypass of a cdA at an open template during DNA replication was examined with the substrates containing a $5^{\prime} R$-cdA or $5^{\prime} S$-cdA with an upstream primer alone annealed with the template strand. (B) Pol $\beta$ DNA synthesis in extending a 3'-terminus dT that base paired with the template cdA lesion during DNA replication was measured with the open template substrates under the experimental conditions. For panels (A) and (B), lanes 1 and 5 represent substrates only. Lanes $2-4$ and lanes $6-8$ correspond to reaction mixtures with $10 \mathrm{nM}, 25 \mathrm{nM}$ and $50 \mathrm{nM}$ pol $\beta$, respectively. (C) Pol $\beta$ bypass of a cdA during Okazaki fragment maturation and BER was examined with the substrates containing a 1 -nt gap opposite a template 5 ' $R$-cdA or 5 ' $S$-cdA and the downstream primer with a 5'-phosphate or a 5'-phosphorylated THF residue. (D) Pol $\beta$ DNA synthesis after a template cdA was bypassed during Okazaki fragment maturation and BER was examined with nick substrates in which the cdA was base paired with a dT. Pol $\beta$ DNA synthesis to extend a dT that was base paired with a template cdA during BER was measured by using nick substrates that contained a downstream primer with a 5'-phosphorylated THF residue. For both panels (C) and (D), lanes 1, 5, 9 and 13 correspond to substrates only. Lanes $2-4,6-8,10-12$ and 14-16 correspond to reaction mixtures with 5,10 and $25 \mathrm{nM}$ pol $\beta$, respectively. Substrates were ${ }^{32} \mathrm{P}$-labeled at the 5 '-end of the upstream primer as indicated. Substrates are illustrated schematically above the gel. 


\subsection{FEN1 cleavage activity during pol $\beta$ lesion bypass of a $5^{\prime} \boldsymbol{R}$-cdA and $5^{\prime} S$-cdA}

FEN1 plays an essential role in both Okazaki fragment maturation and long-patch BER by removing a 5 '-flap created by strand displacement synthesis of replicative DNA polymerases, pol $\delta / \varepsilon$, or repair polymerases such as pol $\beta$ (Balakrishnan and Bambara, 2013; Liu et al., 2004). Removal of an oxidized or reduced deoxyribose phosphate during longpatch BER is dependent on FEN1 flap cleavage activity (Liu and Wilson, 2012; Wilson et al., 2010). Because pol $\beta$ DNA synthesis can result in the formation of a flap, it may affect FEN1 cleavage. To further determine if FEN1 cleavage during Okazaki fragment maturation and BER can be affected by pol $\beta$ lesion bypass of a cdA, we examined FEN1 cleavage activity on the $1 \mathrm{nt}$-gapped substrates containing a template cdA without or with a 5'-THF residue in the absence and presence of pol $\beta$ (Figure 1.4). The results showed that for the substrates with a template 5 'R-cdA, in the absence of pol $\beta$, FEN1 mainly removed 1 or 2 nucleotides from the downstream strand (Figure 1.4, lanes 2 and 8). In the presence of pol $\beta$, FEN1 cleavage was significantly stimulated, and it predominantly removed a flap containing 5 or 6 nucleotides (Figure 1.4, lanes 3 and 9). This indicated that after pol $\beta$ efficiently bypassed a $5^{\prime} R$-cdA, it kept inserting nucleotides to perform strand displacement synthesis, creating a 5-nt or 6-nt downstream flap for FEN1 cleavage. In contrast, for the 5 'S-cdA containing substrates, in the absence of pol $\beta$, FEN1 removed 2 to 4 nucleotides (Figure 1.4, lanes 5 and 11). The presence of pol $\beta$ did not significantly alter FEN1 cleavage activity (Figure 1.4, lanes 6 and 12), indicating that pol $\beta$ failed to efficiently bypass a 5 'S-cdA. This allowed FEN1 to remove more nucleotides than were inserted by pol $\beta$, leaving a gapped intermediate that failed to be sealed by LIG I. This subsequently led to accumulation of a single-strand break intermediate. 


\subsection{A 5'S-cdA lesion disrupts the completion of Okazaki fragment maturation and BER by inhibiting pol $\beta$ lesion bypass synthesis}

When genomic DNA is under the challenge of oxidative stress, oxidative DNA damage including 8-oxoGs and cdPus can be generated simultaneously in DNA in vitro and in vivo at a significant percentage (Belmadoui et al., 2010; Kirkali et al., 2008). Because cdPus including cdA lesions can only be repaired by nucleotide excision repair with low efficiency, this allows the accumulation of the lesions in the genome readily. Thus, 8-oxoGs and cdA lesions may occur simultaneously in the complementary strands of DNA with high frequency, and it is likely that BER of an 8-oxoG and an abasic lesion in one strand of the genome may encounter a cdA base lesion accumulated in the template strand. This could allow pol $\beta$ to perform DNA synthesis to bypass a cdPu lesion, thereby subsequently altering the efficiency of lagging strand maturation and BER. It has also been suggested that BER of a uracil opposite a 5'S-cdA is compromised because the lesion can alter the geometry of duplex DNA (Karwowski et al., 2014). To test this possibility, we reconstituted DNA lagging strand maturation and BER with the substrates containing a 1nt gap opposite a $5^{\prime} R$-cdA or 5' $S$-cdA without or witha 5'-THF residue (Figure 1.5). With the substrates containing a $5^{\prime} R$-cdA, pol $\beta$ exhibited efficient lesion bypass synthesis leading to the production of a significant amount of replication and repair products (Figure 1.5 , lanes $2-4$ and $10-12$ ), indicating that pol $\beta$ bypass of a $5^{\prime} R$-cdA led to the completion of lagging strand maturation and BER. However, with the substrates containing a 5' $S$-cdA, pol $\beta$ only inserted a dT to bypass a template 5'S-cdA and failed to further extend the nucleotide by inserting additional nucleotides (Figure 1.5, lanes 6-8 and 14-16), resulting in the accumulation of a single-strand DNA break intermediate with a template $5^{\prime} S$-cdA. 
These results demonstrated that inefficient bypass of a 5'S-cdA by pol $\beta$ prevented the completion of lagging strand maturation and BER. This further suggests that a 5'S-cdA induced DNA strand distortion more severely than a $5^{\prime} R$-cdA, thereby inhibiting pol $\beta$ lesion bypass and preventing the completion of Okazaki fragment maturation and BER.
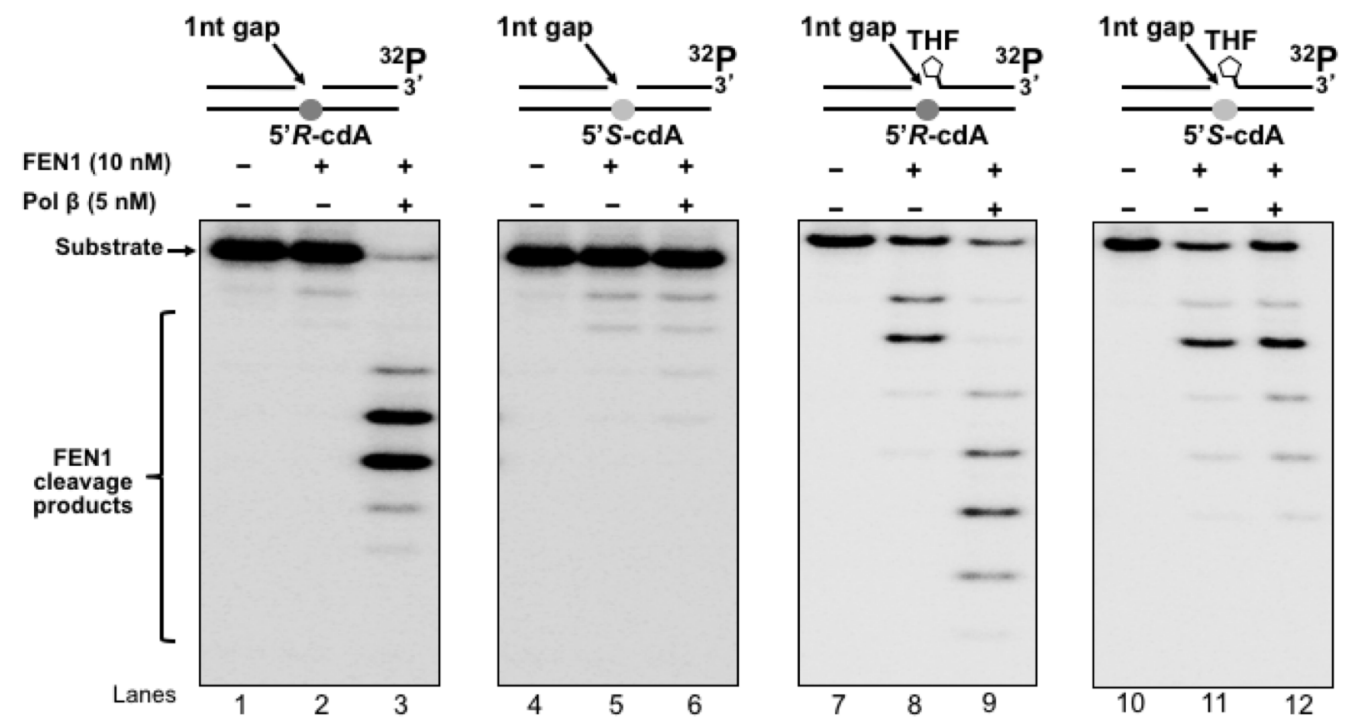

Figure 1.4 FEN1 flap cleavage during pol $\beta$ bypass of a cdA. FEN1 flap cleavage on the substrates containing a 5'-phosphate or 5'-THF residue with a 1-nt gap opposite a template 5 ' $R$-cdA or 5 ' $S$-cdA was examined as described in Section 2. Lanes 1, 4, 7 and 10 represent substrates only. Lanes 2, 5, 8 and 11 correspond to reaction mixtures with 10nM FEN1. Lanes $3,6,9$ and 12 correspond to reaction mixtures with $10 \mathrm{nM}$ FEN1 in the presence of $5 \mathrm{nM}$ pol $\beta$. Substrates were ${ }^{32} \mathrm{P}$-labeled at the 3 '-end of the downstream primer as indicated. Substrates are illustrated schematically above the gel. 


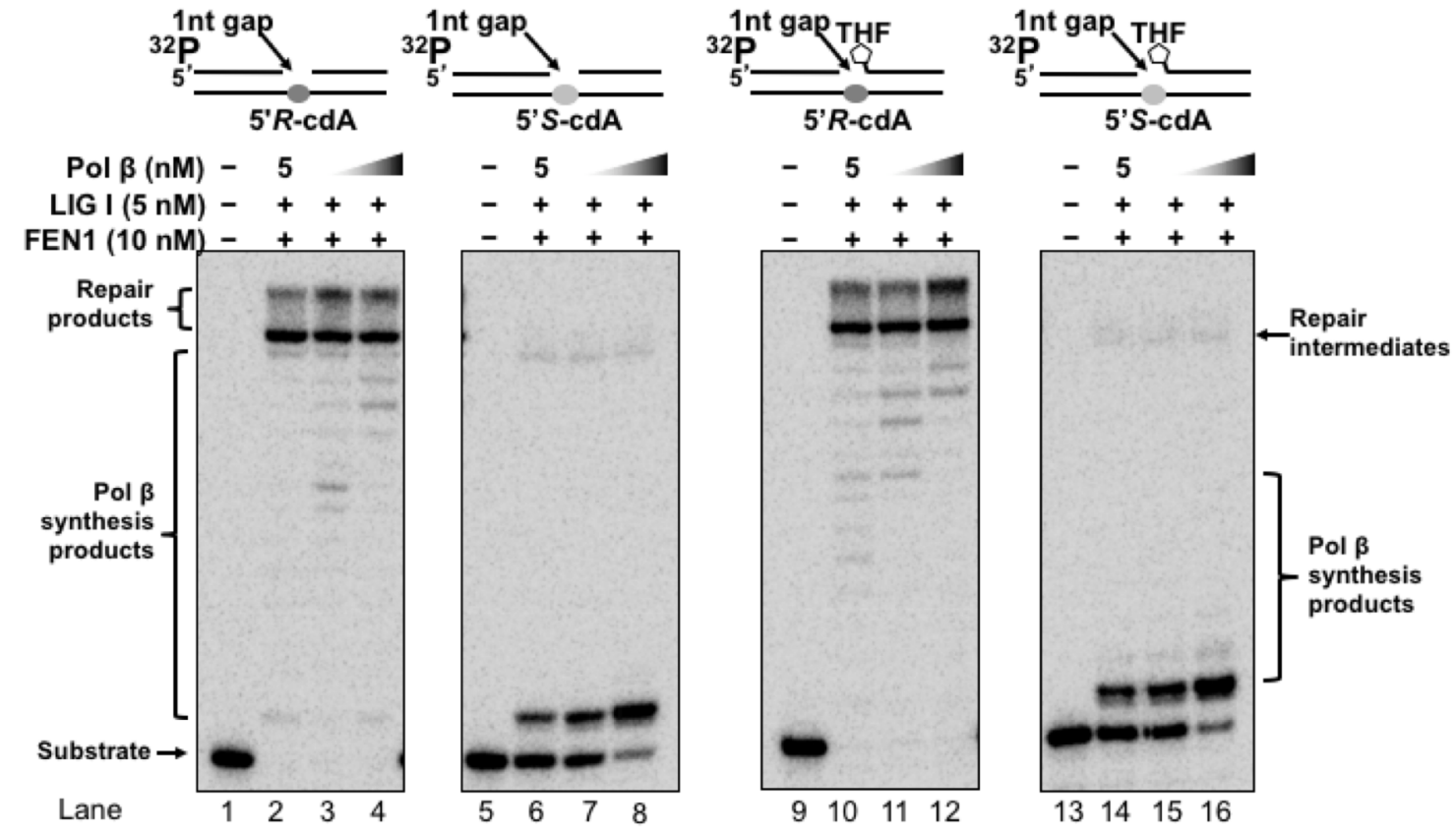

Figure 1.5 Completion of DNA lagging strand maturation and BER through pol $\beta$ bypass of a cdA lesion. Reconstituted DNA lagging strand maturation and BER were performed to determine the effects of pol $\beta$ bypass of a cdA on completion of DNA lagging strand maturation and BER. Substrates containing a 5'-phosphate or 5-THF residue with a 1-nt gap opposite a template 5 ' $R$-cdA or 5 'S-cdA were incubated with indicated BER enzymes at the experimental conditions described in Section 2. Lanes 1, 5, 9 and 13 represent substrates only. Lanes 2-4, 6-8, 10-12 and 14-16 correspond to reconstitution reaction mixtures with $10 \mathrm{nM}$ FEN1 and $5 \mathrm{nM}$ LIG I in the presence of 5,10 and $25 \mathrm{nM}$ pol $\beta$, respectively. Substrates were ${ }^{32} \mathrm{P}$-labeled at the 5 '-end of the upstream primer as indicated. Substrates are illustrated schematically above the gel.

\subsection{Pol $\beta$ can bypass a 5, 8-cdA through nucleotide misinsertion during DNA}

\section{replication and BER}

Previous studies have shown that cdA lesions can completely block DNA synthesis of human replication polymerases such as pol $\delta$, causing replication fork stalling through inducing the distortion of DNA backbone (Kuraoka et al., 2000). To resolve the stalled 
replication fork and restart DNA replication, Y family DNA translesion polymerases are recruited to perform translesion synthesis for bypassing the base lesions (Friedberg et al., 2005). However, a template 5' $S$-cdA can also block the lesion bypass synthesis by Y family polymerases such as pol $\eta$ and pol $\mathrm{l}$, resulting in the accumulation of DNA strand breaks leading to apoptosis (Kuraoka et al., 2001; Pednekar et al., 2014; You et al., 2013). Because pol $\beta$ has also been shown to exhibit translesion synthesis to bypass a DNA base lesion during DNA replication and BER (Bassett et al., 2002a; Chary et al., 2012a; Hashim et al., 1997b; Hoffmann et al., 1996b; Maga et al., 2009b; Servant et al., 2002b; Vaisman and Chaney, 2000b; Villani et al., 2011b) Thus, it is possible that pol $\beta$ can be recruited to perform translesion synthesis to bypass a cdA lesion. However, several studies have shown that pol $\beta$ lesion bypass synthesis can induce a high frequency of mutations in the genome (Bassett et al., 2002a; Batra et al., 2006; Beard et al., 2009; Beard and Wilson, 2014; Chary et al., 2012a; Efrati et al., 1997; Hashim et al., 1997b; Hoffmann et al., 1996b; Servant et al., 2002b; Vaisman and Chaney, 2000b). Furthermore, because of lacking a proofreading 3'-5' exonuclease activity, high levels of pol $\beta$ are associated with high mutation rates in a variety of cancer cells (Canitrot et al., 2000; Scanlon et al., 1989; Srivastava et al., 1999). To investigate whether pol $\beta$ bypass of a cdA can induce mutations in the genome, pol $\beta$ nucleotide insertion during its bypass of a cdA was determined by incubating the open template and gapped substrates that contained a template $5^{\prime} R$-cdA or $5^{\prime} S$-cdA with $5 \mathrm{nM}$ and $50 \mathrm{nM}$ pol $\beta$. The results showed that at a low concentration $(5 \mathrm{nM})$, pol $\beta$ mainly inserted a dT to base pair with a $5^{\prime} R$-cdA with the open template substrates (Figure 1.6A, lane 3). However, the enzyme also inefficiently inserted a dA, dC or $\mathrm{dG}$ to base pair with the 5'R-cdA (Figure 1.6A, lanes 2, 4 and 5). On the other hand, the same concentration of 
pol $\beta$ only inserted dT to base pair with a template $5^{\prime} S$-cdA (Figure 1.6A, lane 8 ) and failed to insert a dA, dC or $\mathrm{dG}$ to bypass the lesion (Figure 1.6A, lane 7 and lanes 9-10). Surprisingly, we found that with the 1-nt gapped substrates without a 5'-THF residue, 5 $\mathrm{nM}$ pol $\beta$ efficiently inserted both $\mathrm{dT}$ and $\mathrm{dC}$ to bypass a template 5 ' $R$-cdA (Figure 1.6A, lanes 13-14) and inefficiently inserted a $\mathrm{dA}$ and $\mathrm{dG}$ to bypass the lesion (Figure 1.6A, lanes 12 and 15). For the 1-nt gap substrate containing a 5' $S$-cdA, $5 \mathrm{nM}$ pol $\beta$ mainly inserted a dT to bypass the lesion (Figure 1.6A, lane 18). It also inserted a dA with a very low efficiency (Figure 1.6A, lane 17), but it failed to insert dC and dG (Figure 1.6A, lanes 1920). For the 1-nt gap substrates with a 5'-THF residue, $5 \mathrm{nM}$ pol $\beta$ predominantly inserted a dT and dC to bypass a $5{ }^{\prime} R$-cdA (Figure 1.6A, lanes 23-24), although the enzyme also managed to insert a dA and dG inefficiently to bypass the lesion (Figure 1.6A, lanes 22 and 25). The same concentration of pol $\beta$ inefficiently inserted a dT to bypass a 5' $S$-cdA (Figure 1.6A, lane 28) and failed to insert other types of nucleotides to bypass the lesion (Figure 1.6A, lanes 27, 29 and 30). At a high concentration of $50 \mathrm{nM}$, pol $\beta$ misinsertion in bypassing a cdA lesion with all the substrates was significantly increased (Figure 1.6B, lanes 2, 4-5, lanes 12, 14-15, lanes 17, 19-20, and lanes 22, 24-25, 27). The high concentrations also significantly increased pol $\beta$ correct nucleotide insertion in bypassing a 5'S-cdA (Figure 1.6B, lanes 8, 18 and 28). However, the high concentrations of pol $\beta$ failed to increase the pol $\beta$ misinsertion of a dA to pair with a 5'S-cdA with the open template substrate (Figure 1.6B, lane 7). The results indicated that at both a low and high concentration, pol $\beta$ efficiently misinserted $\mathrm{dC}$ to bypass a $5^{\prime} R$-cdA during DNA lagging strand maturation and BER. However, a high concentration of pol $\beta$ also significantly promoted its misinsertation of $\mathrm{dA}$ and $\mathrm{dG}$ during its lesion bypass. The results further 
suggest that pol $\beta$ bypass of a 5'R-cdA or 5' $S$-cdA lesion during replication and BER may

be mutagenic, especially in the presence of a high level of pol $\beta$.
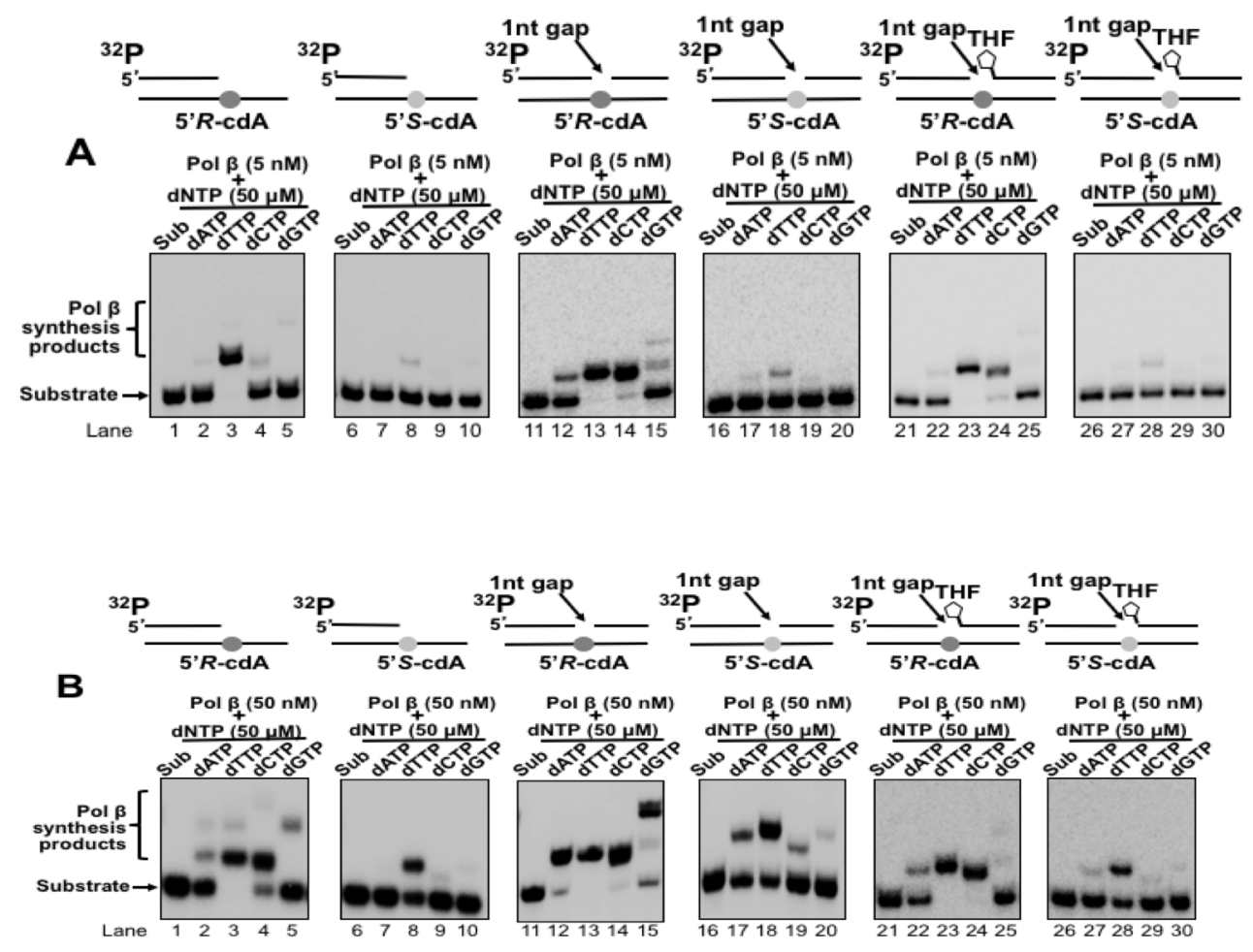

Figure 1.6 Pol $\beta$ nucleotide insertions in bypassing a cdA lesion during DNA replication and BER. Pol $\beta$ nucleotide insertion in bypassing a cdA during DNA lagging strand maturation and BER was examined by incubating the open-template substrates and 1-nt gap substrates that contained a downstream 5'-phosphate or 5'-THF residue and a template 5 ' $R$-cdA or 5 ' $S$-cdA with $5 \mathrm{nM}$ (A) or $50 \mathrm{nM}$ (B) pol $\beta$ in the presence of $50 \mathrm{nM}$ dATP or dTTP or dCTP or dGTP, respectively, under the experimental conditions described in Section 2. In both panels, lanes 1, 6, $11,16,21$ and 26 represent substrates only. Lanes $2-5,7-10,12-15,17-20,22-25$ and $27-30$ correspond to reaction mixtures with $5 \mathrm{nM}$ or $50 \mathrm{nM}$ pol $\beta$ in the presence of $50 \mathrm{nM} \mathrm{dATP}$, dTTP, $\mathrm{dCTP}$ or dGTP. Substrates were ${ }^{32} \mathrm{P}$-labeled at the 5 '-end of the upstream primer as indicated. Substrates are illustrated schematically above the gel. 


\section{DISCUSSION}

In this study, for the first time we discovered that pol $\beta$ played an important role in bypassing a cdA lesion during DNA replication and BER (Figure 2). We provided the first evidence that pol $\beta$ efficiently bypassed a 5' $R$-cdA via multi-nucleotide insertions, whereas the enzyme mainly inserted $1 \mathrm{nt}$ to bypass a 5 ' $S$-cdA lesion and failed to further perform its multi-nucleotide lesion bypass DNA synthesis (Figures 2-3). This resulted in disruption of its DNA synthesis in bypassing a 5'S-cdA during DNA replication and BER. Furthermore, we discovered that a $5^{\prime} S$-cdA on the template strand also slightly facilitated FEN1 removal of a long flap (Figure 1.4), suggesting that the lesion induced a distortion on the DNA structure, thereby promoting dissociation of the downstream DNA strand from its template. This further resulted in the formation of a flap that can be efficiently cleaved by FEN1 leaving gapped DNA thereby inhibiting the completion of DNA replication and repair and leading to accumulation of single-strand intermediates (Figure 1.5). Moreover, we found that during DNA replication and BER, a low concentration of pol $\beta$ inserted both a correct and incorrect nucleotide to bypass a cdA, whereas a high concentration of pol $\beta$ stimulated its nucleotide misincorporation in bypassing a cdA (Figure 1.6). Our results support a model in which a 5' $R$-cdA induced by ROS during DNA replication or BER can be efficiently bypassed by pol $\beta$. In the presence of a low concentration of pol $\beta$, a correct nucleotide is inserted to base pair with the lesion. Pol $\beta$ continues to perform strand displacement synthesis, creating a long flap which is subsequently cleaved by FEN1. This leads to the production of a nicked DNA that is sealed by LIG I, resulting in no mutation in the replication and repair products (Figure 1.7, sub-pathway 1, left). However, both a low and high concentration of pol $\beta$ can also insert a $\mathrm{dC}$ or $\mathrm{dG}$ or $\mathrm{dA}$ to bypass a $5^{\prime} R$-cdA, 
resulting in mutations (Figure 1.7, sub-pathway 1, right). On the other hand, a template 5' $S$-cdA induces the DNA distortion promoting dissociation of the downstream strand from its template strand resulting in the formation of a small flap. Pol $\beta$ inefficiently inserts a dT to base pair with a template 5' $S$-cdA and stalls (Figure 1.7, subpathway 2). Subsequently, this allows FEN1 to remove more nucleotides than pol $\beta$ synthesizes, resulting in accumulation of single-strand DNA break intermediates (Figure 1.7, subpathway 2). 


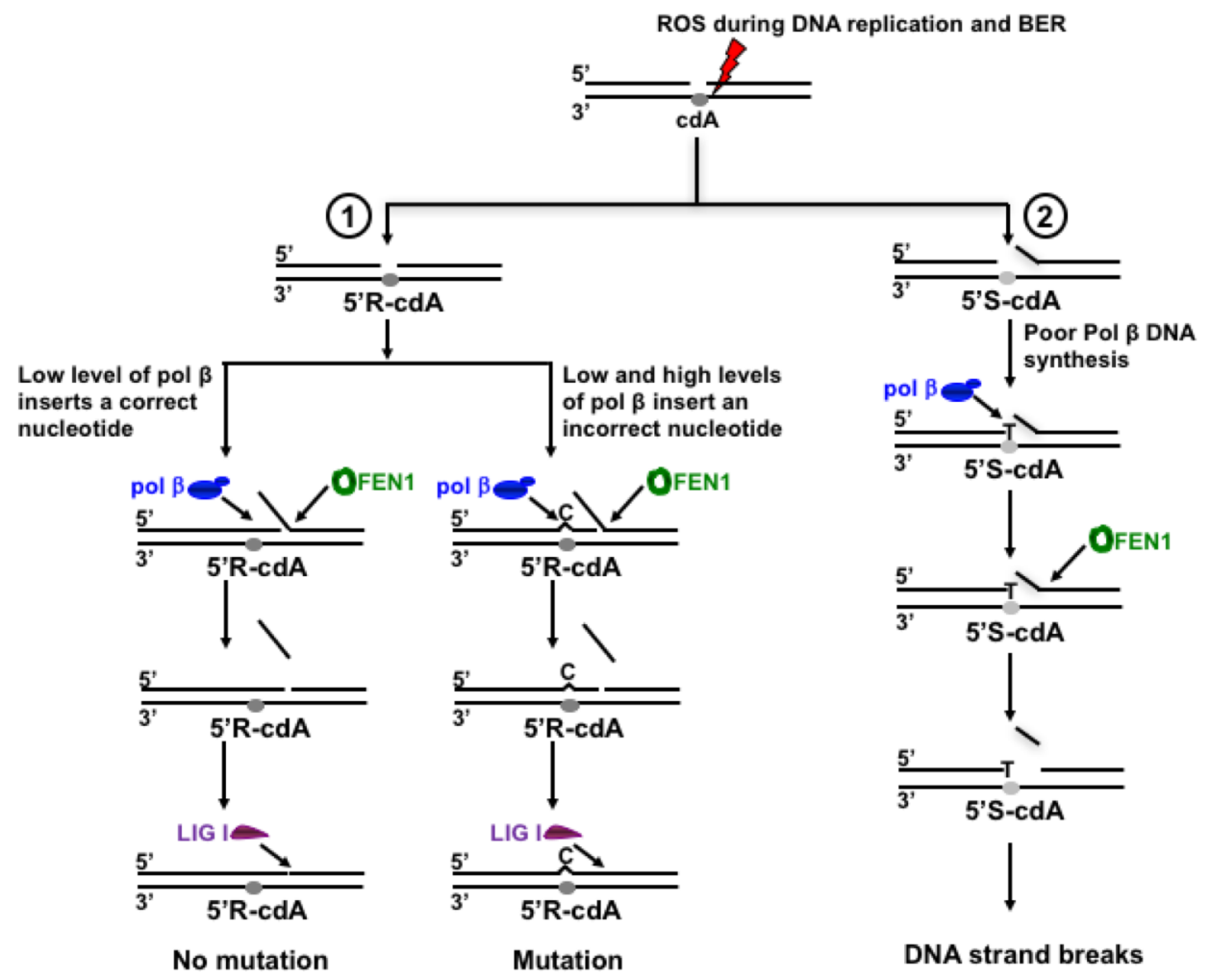

Figure 1.7 Pol $\beta$ bypass of a cdA lesion leads to nucleotide misinsertion and DNA strand breaks during DNA replication and BER. Reactive oxygen species (ROS) can result in oxidized DNA lesion, including cdAs with two different configurations, 5'R-cdA or 5'S-cdA., during DNA replication and BER. A $5^{\prime} R$-cdA can be efficiently bypassed by pol $\beta$. In the presence of low concentration of pol $\beta$, a correct nucleotide can be inserted to base pair with a template cdA lesion. Pol $\beta$ can continue to perform strand displacement synthesis, resulting in the formation of a long flap which is subsequently cleaved by FEN1. This leads to a nicked DNA for LIG I to seal, resulting in no mutation in the replication and repaired product (Subpathway 1 , left). However, a low level of pol $\beta$ can also insert a $\mathrm{dC}$ to base pair a cdA, and the nucleotide misinsertion is promoted at a high level of pol $\beta$ (Sub-pathway 1, right). This subsequently results in mutation during DNA replication and BER. On the other hand, a template 5'S-cdA can induce DNA distortion that promotes dissociation of the downstream strand from its template strand, thereby leading to the formation of an intermediate with a gap and short flap (Sub-pathway 2). The lesion also strongly inhibits pol $\beta$ lesion bypass synthesis as well as extension of the lesion bypass intermediate (Sub-pathway 2). Subsequently, FEN1 cleaves the flap removing more nucleotides than pol $\beta$ synthesizes, causing accumulation of gapped intermediates that can further lead to DNA strand break intermediates (Sub-pathway 2).

Employing the cell extract-based in vitro approach, we have demonstrated the involvement of pol $\beta$ in cdA lesion bypass during DNA replication and repair explicitly. 
Our results obtained from pol $\beta^{+/+}$and pol $\beta^{-/-}$MEFs indicate that pol $\beta$ plays an important role in bypassing cdA lesions (Figure 1.2). This is also supported by a study from the Wilson group showing that pol $\beta^{-/}$MEFs exhibited hypersensitivity to the oxidative DNA damaging agent, hydrogen peroxide than pol $\beta^{+/+}$MEFs (Horton et al., 2002). Because hydrogen peroxide is the precursor for generating hydroxyl radicals that can subsequently lead to cdA lesions, this further indicates that the hypersensitivity of pol $\beta^{-/-}$MEFs to hydrogen peroxide results from the accumulation of oxidized DNA lesions including cdA lesions and DNA strand breaks caused by the deficiency of lesion bypass in the absence of pol $\beta$.

Our results indicate that pol $\beta$ efficiently bypassed a $5^{\prime} R$-cdA (Figure 1-2). However, it also inserted a significant amount of incorrect nucleotides to bypass the lesion at a low concentration $(5 \mathrm{nM})$, and this was significantly stimulated at a high concentration $(50 \mathrm{nM})$ (Figure 6). Given the fact that in mammalian cells, during BER of oxidative DNA damage, a large number of pol $\beta$ molecules can be recruited to damaged sites resulting in a high concentration of the enzyme that is accumulated at oxidized base lesions (Lan et al., 2004), it is likely that pol $\beta$ nucleotide misinsertions can frequently occur during its bypass of a cdA in cells. This can then lead to a high frequency of mutations in genomic DNA. Our results are also consistent with previous findings showing that in human cancer cells, pol $\beta$ protein level has been increased significantly compared with normal cells, thereby inducing a high frequency of mutations (Canitrot et al., 2000; Scanlon et al., 1989; Srivastava et al., 1999). Moreover, because of the lack of an intrinsic 3'-5' exonuclease, pol $\beta$ lesion bypass can induce more mutations in the genome than replication polymerases through its nucleotide misinsertion during its lesion bypass synthesis (Beard et al., 2014). 
It is conceivable that an extrinsic 3 ' -5 ' exonuclease that can remove a mismatched base may improve pol $\beta$ fidelity during its bypass of a template cdA. It has been reported that in addition to its cleavage of an abasic site during BER, APE1 also has 3'-5' exonuclease activity that can remove mismatched bases at the 3'-end in nicked or gapped DNA (Chou and Cheng, 2002; Parsons et al., 2005). Previous studies have also found that p53 can efficiently remove a misinserted base prior to the extension of the mismatched 3'-terminus by a DNA polymerase (Bakhanashvili, 2001; Rahav, 2013). Because APE1 can form a complex with pol $\beta$ (Liu et al., 2007b), and p53 can interact with pol $\beta$ (Zhou et al., 2001), it is possible that these proteins may cooperate with pol $\beta$ to remove a misinserted nucleotide incorporated by pol $\beta$ during its bypass of a cdA lesion. It is of interest to determine if APE1 and p53 can remove a mismatched base that pairs with a template $5^{\prime} R$ cdA, improving pol $\beta$ fidelity during its lesion bypass synthesis of a 5',8-cyclopurine-2'deoxynucleoside.

Our results also demonstrated that bypass of a $5^{\prime} S$-cdA is a challenge for pol $\beta$ (Figures1.2, 1.3). We found that pol $\beta$ failed to extend the 3 '-terminus after it inserted one nucleotide to base pair with a $5^{\prime} S$-cdA. This subsequently results in the accumulation of single-strand break intermediates that may subsequently lead to double-strand breaks and cell death. Previous studies have shown that pol $\beta$ DNA synthesis in bypassing an AP site during Okazaki fragment maturation can be promoted by proliferating cell nuclear antigen (PCNA) and replication protein A (RPA) (Maga et al., 2009b). It has been also shown that through coordination with FEN1 and RPA, pol $\beta$ strand displacement synthesis activity is also facilitated after it bypasses an abasic site (Maga et al., 2009b). It is conceivable that the replication and repair cofactors that can facilitate pol $\beta$ lesion bypass activity and primer 
extension may facilitate pol $\beta$ bypass of a $5^{\prime} S$-cdA as well as its extension of the lesion bypass intermediates. Therefore, it is of interest to investigate how BER cofactors, PCNA and RPA may also facilitate pol $\beta$ bypass of a 5 ' $S$-cdA, preventing the formation of DNA strand break intermediates and cell death.

In summary, we identified that pol $\beta$ played an important role in bypassing a template cdA lesion during DNA replication and BER. We provided the first evidence that pol $\beta$ can efficiently bypass a $5^{\prime} R$-cdA, but inefficiently bypass a $5^{\prime} S$-cdA, resulting in the incompletion of DNA replication and repair and accumulation of strand break intermediates. Moreover, we found that pol $\beta$ inserted an incorrect nucleotide to bypass a $\operatorname{cdA}$, and this was promoted by a high concentration of pol $\beta$, indicating that the fidelity of pol $\beta$ bypass of a cdA lesion during DNA replication and BER can be modulated by the concentrations of pol $\beta$. This may further result in mutations in the genome. In addition, we demonstrated that a template 5'S-cdA severely inhibited pol $\beta$ lesion bypass synthesis and induced dissociation of the downstream strand from the template strand resulting in the formation of a short flap (Figure 1.4 and Figure 7 sub-pathway 2). This subsequently led to poor pol $\beta$ DNA synthesis along with relatively efficient FEN1 flap cleavage on the downstream strand, resulting in the accumulation of strand break intermediates (Figure 1.5, lanes 6-8 and lanes 14-16). Our results reveal that pol $\beta$ bypass of a cdA can result in nucleotide misinsertions and DNA strand breaks during DNA replication and BER, thereby resulting in genome instability. 


\title{
CHAPTER 2
}

\section{AP ENDONUCLEASE 1 COMBATS THE EXTENSION OF A T/G MISMATCH BY DNA POLYMERASE $\beta$ TO PREVENT MUTATIONS IN CpGs DURING BASE EXCISION REPAIR}

\begin{abstract}
Dynamics of DNA methylation and demethylation at $\mathrm{CpG}$ clusters are involved in gene regulation. $\mathrm{CpG}$ clusters have been identified as hot spots of mutagenesis because of their susceptibility to oxidative DNA damage. Damaged Cs and Gs at CpGs can disrupt a normal DNA methylation pattern through modulation of DNA methylation and demethylation, leading to mutations and deregulation of gene expression. DNA base excision repair (BER) plays a dual role of repairing oxidative DNA damage and mediating an active DNA demethylation pathway on $\mathrm{CpG}$ clusters through removal of a $\mathrm{T} / \mathrm{G}$ mismatch resulting from deamination of a $5 \mathrm{mC}$ adjacent to a guanine that can be simultaneously damaged by oxidative stress. However, it remains unknown how BER processes clustered lesions in CpGs and what are the consequences from the repair of these lesions. In the present study, we examined BER of an abasic lesion next to a DNA demethylation intermediate, the $\mathrm{T} / \mathrm{G}$ mismatch in a $\mathrm{CpG}$ dinucleotide, and its effect on the integrity of CpGs. Surprisingly, we found that the abasic lesion completely abolished the activity of thymine DNA glycosylase (TDG) for removing the mismatched T. However, we found that APE1 could still efficiently incise the abasic lesion leaving a 3-terminus mismatched $\mathrm{T}$, which was subsequently extended by pol $\beta$. This in turn resulted in a $\mathrm{C}$ to $\mathrm{T}$ transition mutation. Interestingly, we also found that APE1 3' -5 ' exonuclease activity efficiently removed the mismatched $T$, thereby preventing pol $\beta$ extension of the
\end{abstract}


mismatched nucleotide and the resulting mutation. Our results demonstrate a crucial role of APE1 3'-5' exonuclease activity in combating mutations in CpG clusters caused by an intermediate of DNA demethylation during BER.

\section{INTRODUCTION}

Dynamics of cytosine methylation, i.e., formation of 5-methyl cytosine $(5 \mathrm{mC})$ and demethylation of $5 \mathrm{mC}$ in $\mathrm{CpG}$ islands (CGIs), in the mammalian genome are actively involved in the regulation of gene expression, inactivation of the $\mathrm{X}$ chromosome, and gene imprinting, among others (Bhutani et al., 2011; Chen and Riggs, 2011; Jones, 2012; Jones and Takai, 2001). It is estimated that only approximately $1-2 \%$ of cytosines in the mammalian genome are unmethylated (Bird et al., 1985; Bird, 1986; Illingworth et al., 2008; Suzuki and Bird, 2008), whereas 60-90\% of CpGs are methylated (Jones and Takai, 2001; Siegfried and Cedar, 1997). The DNA methylation pattern regulates gene transcription by facilitating or blocking access of transcription factors to gene promoter or transcribed regions directly (Becker et al., 1987; Tate and Bird, 1993), or by modulating the recruitment of methyl CpG binding proteins (Lewis et al., 1992; Meehan et al., 1989; Tate et al., 1996), as well as by altering histone modifications and chromatin structures (Berger, 2002; Cheung et al., 2000; Clark et al., 1997; Hendrich and Bird, 1998). A normal DNA methylation pattern is essential for maintaining the homeostasis of gene expression and cellular function, whereas an aberrant DNA methylation pattern is associated with the onset and progression of many diseases (Jones and Baylin, 2007; Robertson, 2005; Valinluck and Sowers, 2007). It has been found that hypermethylation of CpGs on tumor suppressor genes (TSGs) (Rideout et al., 1990; Stirzaker et al., 1997) and hypomethylation 
of $\mathrm{CpGs}$ on oncogenes result in deregulation of expression of the genes leading to the development of cancer (Jones, 2012).

5-methylcytosine $(5 \mathrm{mC})$ can be removed by a process called DNA demethylation, which can occur either passively or actively. Passive DNA demethylation results from the failure of DNA methyltransferases to methylate a cytosine during DNA replication and cell division (Bhutani et al., 2011). Passive DNA demethylation may also result from the loss or substitution of cytosines by DNA damage and repair in the context of CpGs. Active DNA demethylation is mediated by sequential enzymatic reactions that can occur independent of DNA replication and cell division (Bhutani et al., 2011; Chen and Riggs, 2011; Cortellino et al., 2011; Zhu, 2009). Removal of a $5 \mathrm{mC}$ by active DNA demethylation is initiated by modifications of the nucleotide through several types of enzymatic reactions, including hydroxylation, deamination and oxidation that convert the $5 \mathrm{mC}$ into a modified base or base lesion that is recognized and cleaved by DNA glycosylases (Bhutani et al., 2011; Chen and Riggs, 2011) which subsequently leads to replacement of the 5mC through the DNA base excision repair (BER) pathway (Bhutani et al., 2011; Chen and Riggs, 2011; Cortellino et al., 2011). It has been shown that BER-mediated active DNA demethylation is accomplished through several pathways depending on the type of modifications of $5 \mathrm{mC}$ that are removed by different types of DNA glycosylases (Bhutani et al., 2011; Chen and Riggs, 2011; Gehring et al., 2009; Zhu, 2009). One of the pathways is initiated by a direct deamination of $5 \mathrm{mC}$ by activation-induced cytidine deaminase (AID) that converts a $5 \mathrm{mC}$ to a thymine, resulting in a T/G mismatch (Morgan et al., 2004). The mismatched $\mathrm{T}$ can be subsequently excised by thymine DNA glycosylase (TDG) (Cortellino et al., 2011; Neddermann and Jiricny, 1993, 1994; Zhang et al., 2012), leaving an abasic site that is then 
subjected to BER which ultimately leads to replacement of the $5 \mathrm{mC}$ with an unmethylated C (Bhutani et al., 2011). Another BER-mediated DNA demethylation pathway is initiated by single-strand-selective monofunctional uracil-DNA glycosylase 1 (SMUG1) that removes a 5 -formyluracil generated from oxidation of a $5 \mathrm{mC}$ by a family of enzymes called Ten Eleven Translocation (TET), a methylcytosine dioxygenase (He et al., 2011; Ito et al., 2011; Williams et al., 2011), or from oxidative DNA damage induced by hydroxyl radicals (Bjelland et al., 1995; Masaoka et al., 2003). Thus, in mammalian cells, DNA base damage and BER are strategically used as a mechanism for both passive and active DNA demethylation (Gehring et al., 2009).

While the cytosine in a CpG dinucleotide is a substrate for DNA methylation and demethylation, the neighboring 3'-guanine is a hotspot of oxidative DNA damage. As the most abundant form of oxidative DNA damage in mammalian cells, 8-oxoguanine (8-oxoG) readily accumulates in $\mathrm{CpGs}$ and may affect the integrity of CpGs by modulating the production and processing of DNA demethylation intermediates. It has been found that 8oxoG can cause accumulation of $\mathrm{T} / \mathrm{G}$ mismatched base pairs when it occurs adjacent to $5 \mathrm{mC}$ by inhibiting the removal of the mismatched $\mathrm{T}$ (Sassa et al., 2014). Moreover, when BER of 8-oxoG encounters active DNA demethylation, repair of the lesion may be affected by a DNA demethylation intermediate which has been supported by a recent study from the Wilson group showing that the efficiency of removal of 8-oxoG by OGG1 was significantly reduced by an adjacent 5'-T/G mismatch, a DNA demethylation intermediate generated from deamination of $5 \mathrm{mC}$ in CpGs (Sassa et al., 2014). This subsequently inhibited the completion of BER. This suggests that a base lesion interferes with an essential step of BER-mediated DNA demethylation, thereby compromising the efficiency 
and fidelity of DNA demethylation in CpGs. These studies further suggest that BER of oxidative DNA damage in CpGs and DNA demethylation intermediates has to be properly coordinated to maintain the integrity and fidelity of CpGs. However, it remains unknown how the fidelity of CpGs may be maintained by BER in coordinating its dual function in repairing a base lesion as well as in mediating active DNA demethylation. In the current study, we explored how an abasic lesion that occurs in a $\mathrm{CpG}$ at the 3 '-side of a $\mathrm{T} / \mathrm{G}$ mismatch could affect the integrity of a $\mathrm{CpG}$ dinucleotide by modulating the removal of the mismatched $\mathrm{T}$ during BER, and how BER may coordinate the removal of the base lesion and maintenance of the fidelity of $\mathrm{CpG}$ dinucleotides. For the first time, we show that the abasic lesion completely inhibited the removal of its adjacent 5'-mismatched $\mathrm{T}$ by TDG leading to accumulation of the mismatched nucleotide. We show that DNA polymerase $\beta$ (pol $\beta$ ) readily tolerated the mismatched $\mathrm{T}$ and efficiently extended the mismatched nucleotide, allowing the sustainment of the $\mathrm{T} / \mathrm{G}$ mismatch and leading to a $\mathrm{C}$ to $\mathrm{T}$ transition mutation during BER. Interestingly, we discovered that AP endonuclease 1 (APE1) 3'-5' exonuclease efficiently removed the mismatched $\mathrm{T}$, thereby preventing the mutation. Our results indicate that APE1 3'-5' exonuclease plays a crucial role in maintaining the integrity of $\mathrm{CpGs}$ during BER and DNA demethylation. This demonstrates that the coordination between BER enzymes effectively removes a 3'-mismatched $\mathrm{T}$, thus preventing mutations that result from BER and BER-mediated active DNA demethylation. Our study provides new insights into the molecular mechanisms underlying the roles of BER in preventing $\mathrm{T} / \mathrm{G}$ mismatches and sustaining the integrity of $\mathrm{CpGs}$ during BER and active DNA demethylation. 


\section{MATERIALS AND METHODS}

\subsection{Materials}

Oligonucleotides were synthesized by Integrated DNA Technologies Inc. (Coralville, IA). The radionucleotides $\left[\gamma_{-}{ }^{32} \mathrm{P}\right]$ ATP $(6000 \mathrm{Ci} / \mathrm{mmol})$ and cordycepin $5 '-$ triphosphate $3^{\prime}-\left[\alpha^{32} \mathrm{P}\right](5000 \mathrm{Ci} / \mathrm{mmol})$ were purchased from PerkinElmer Inc. (Boston, MA).Micro Bio-Spin 6 chromatography columns were from Bio-Rad (Hercules, CA). T4 polynucleotide kinase (PNK) and terminal deoxynucleotidyl transferase (TdT) were from Fermentas (Glen Burnie, MD). Adenosine 5'-triphosphate (ATP) (100 mM) was from USB (Cleveland, Ohio). Purified thymine DNA glycosylase (TDG) was from Enzymax, LLC (Lexington, Kentucky). Purified APE1, pol $\beta$, flap endonuclease 1 (FEN1) and DNA ligase I (LIG I) were generous gifts from Dr. Samuel H. Wilson at the Genome Integrity and Structural Biology Laboratory, National Institute of Environmental Health Science/National Institutes of Health (NIEHS), Research Triangle Park, NC. All other reagents were from Thermo Fisher Scientific (Pittsburgh, PA) and Sigma-Aldrich (St. Louis, MO).

\subsection{Oligonucleotide substrates}

An oligonucleotide substrate containing a $\mathrm{T} / \mathrm{G}$ mismatched base pair adjacent to a $\mathrm{G} / \mathrm{C}$ matched base pair or a tetrahydrofuran (THF), an abasic site analog, was designed to mimic the intermediates resulting from deamination of a 5-methylcytosine by AID in a CpG dinucleotide with an undamaged $\mathrm{G}$ or an abasic lesion (AP site) that substituted G. Substrates containing a T/G mismatch at the upstream primer and a 5' $-\mathrm{THF}$ residue at the downstream primer were designed to mimic the BER intermediates containing an oxidized AP site adjacent to a T/G mismatch that is 5'-incised by APE1 opposite to a template $\mathrm{C}$ or 
T. The substrate containing an intact or preincised AP site opposite to a template $\mathrm{C}$ and adjacent to a $\mathrm{C} / \mathrm{G}$ matched base pair was used as the control. Substrates for measuring TDG activity were constructed by annealing the strand containing a $\mathrm{T}$ with the template strand containing a $\mathrm{G}$ that was base paired with the $\mathrm{T}$ at a molar ratio of 1:1.5. Substrates for measuring BER enzymatic activity were constructed by annealing the upstream primer with a 3'-T that mispaired with a template $\mathrm{G}$ and the downstream primer with a 5'-THF residue to the template strand at a molar ratio of 1:1:2. The sequences of the oligonucleotide substrates are listed in Table 2.1.

Table 2.1 Sequences of the oligonucleotide substrates

\begin{tabular}{|c|c|c|}
\hline Oligonucleotides & $\mathrm{nt}$ & Sequence $\left(5^{\prime}-3^{\prime}\right)$ \\
\hline \multicolumn{3}{|l|}{ Damaged Strands } \\
\hline D1 & 31 & $\begin{array}{l}\text { GCA CCG GAT CCG CAC CTG GCA TCA GCT GCA } \\
\text { G }\end{array}$ \\
\hline D2 & 30 & $\begin{array}{l}\text { GCA CCG GAT CCG CAC CTF GCA TCA GCT GCA } \\
\text { G }\end{array}$ \\
\hline \multicolumn{3}{|l|}{ Upstream Strands } \\
\hline US1 & 37 & $\begin{array}{l}\text { CGA GTC ATC TAG CAT CCG TAG CAC CGG ATC } \\
\text { CGC ACC C }\end{array}$ \\
\hline US2 & 37 & $\begin{array}{l}\text { CGA GTC ATC TAG CAT CCG TAG CAC CGG ATC } \\
\text { CGC ACC T }\end{array}$ \\
\hline \multicolumn{3}{|l|}{ Downstream } \\
\hline DS1 & 33 & $\begin{array}{l}\text { pF-GCA TCA GCT GCA GTA CGT AGA CTT ACT } \\
\text { CAT TGC }\end{array}$ \\
\hline \multicolumn{3}{|l|}{ Template Strands } \\
\hline $\mathrm{T} 1$ & 31 & $\begin{array}{l}\text { CTG CAG CTG ATG CCG GGT GCG GAT CCG GTG } \\
\text { C }\end{array}$ \\
\hline $\mathrm{T} 2$ & 71 & $\begin{array}{l}\text { GCA ATG AGT AAG TCT ACG TAC TGC AGC TGA } \\
\text { TGC CGG GTG CGG ATC CGG TGC TAC GGA TGC } \\
\text { TAG ATG ACT CG }\end{array}$ \\
\hline $\mathrm{T} 3$ & 71 & $\begin{array}{l}\text { GCA ATG AGT AAG TCT ACG TAC TGC AGC TGA } \\
\text { TGC TGG GTG CGG ATC CGG TGC TAC GGA TGC } \\
\text { TAG ATG ACT CG }\end{array}$ \\
\hline
\end{tabular}

${ }^{a}$ The damaged base is in boldface. $\mathrm{F}$, tetrahydofuran. 


\subsection{Measurement of TDG activity in removing a T/G mismatch adjacent to a THF residue, an analogue of an $\mathrm{AP}$ site}

The TDG activity for removing the $\mathrm{T}$ from a $\mathrm{T} / \mathrm{G}$ mismatch adjacent to a THF residue was measured by incubating increasing concentrations of TDG from $50 \mathrm{nM}$ to 70 nM with $25 \mathrm{nM}$ substrate containing a T/G mismatch with or without the THF residue. The activity was examined at $37^{\circ} \mathrm{C}$ for $30 \mathrm{~min}$ in $10 \mu \mathrm{l}$ reaction buffer with $50 \mathrm{mM}$ Tris- $\mathrm{HCl}$, $\mathrm{pH}$ 7.5, $1 \mathrm{mM}$ EDTA, $1 \mathrm{mM}$ DTT, $0.1 \mathrm{mg} / \mathrm{ml} \mathrm{BSA}$, and 0.01\% Nonidet P-40. Reactions were terminated by transferring to $95^{\circ} \mathrm{C}$ for $5 \mathrm{~min}$. The reaction mixture was then treated with $0.1 \mathrm{M} \mathrm{NaOH}$ and denatured at $95^{\circ} \mathrm{C}$ for $10 \mathrm{~min}$ in buffer containing $95 \%$ formamide and $10 \mathrm{mM}$ EDTA. Substrates and products were separated by $15 \%$ urea-denaturing polyacrylamide gel electrophoresis (PAGE) and detected by a Pharos FX Plus Imager (Biorad Laboratories, Hercules, CA).

\subsection{Measurement of APE1 activity in removing an abasic site adjacent to a T/G mismatch}

The APE1 incision of the THF residue, an abasic site analog, that is adjacent to $5 \mathrm{mC}$ or a $\mathrm{T} / \mathrm{G}$ mismatch, was measured at $37{ }^{\circ} \mathrm{C}$ in a $20 \mu \mathrm{l}$ reaction mixture containing 50 $\mathrm{mM}$ Tris- $\mathrm{HCl}, \mathrm{pH}$ 7.5, $50 \mathrm{mM} \mathrm{KCl,} 0.1 \mathrm{mM}$ EDTA, $0.1 \mathrm{mg} / \mathrm{ml} \mathrm{BSA,} 5 \mathrm{mM} \mathrm{MgCl}$, and 0.01\% Nonidet P-40. APE1 activity was examined by incubating $5 \mathrm{nM}$ APE1 with $25 \mathrm{nM}$ substrate containing the $\mathrm{THF}$ residue adjacent to a mismatched $\mathrm{T}$ or matched $\mathrm{C} / \mathrm{G}$ in the absence and presence of increasing concentrations of TDG (10, 25, 50 and $70 \mathrm{nM})$. Reactions were terminated by transferring to $95{ }^{\circ} \mathrm{C}$ for 5 min in stopping buffer containing $95 \%$ formamide and $10 \mathrm{mM}$ EDTA. Substrates and products were separated by $15 \%$ ureadenaturing PAGE and detected by a Pharos FX Plus Imager. 


\subsection{Measurement of pol $\beta$ DNA synthesis activity in extending the 3'-terminus mismatched $T$ of a $T / G$ mismatch}

The Pol $\beta$ DNA synthesis on a single-nucleotide gapped substrate to extend a 3'mismatched $\mathrm{T}$ or 3'-matched $\mathrm{C}$ was measured using a one-nucleotide gapped substrate containing a $\mathrm{T} / \mathrm{G}$ mismatch or a $\mathrm{C} / \mathrm{G}$ match at the 3'-terminus of the upstream primer and a 5'-THF residue at the downstream primer. Enzymatic reactions were performed by incubating $25 \mathrm{nM}$ substrate with various concentrations of pol $\beta$ at $37{ }^{\circ} \mathrm{C}$ for $15 \mathrm{~min}$ in a $10 \mu \mathrm{l}$ reaction mixture containing BER buffer $(50 \mathrm{mM}$ Tris- $\mathrm{HCl}, \mathrm{pH} 7.5,50 \mathrm{mM} \mathrm{KCl}, 0.1$ mM EDTA, $0.1 \mathrm{mg} / \mathrm{ml} \mathrm{BSA,} \mathrm{0.01 \%} \mathrm{Nonidet} \mathrm{P-40,} 5 \mathrm{mM} \mathrm{MgCl}_{2}$ ). APE1 3'-5' exonuclease activity of removing the $\mathrm{T}$ from the 3'-T/G mismatch was measured in BER buffer at 37 ${ }^{\circ} \mathrm{C}$ for 15 min. FEN1 cleavage activity on the THF residue was examined in BER reaction buffer with $50 \mu \mathrm{M}$ dNTPs in the absence or presence of $5 \mathrm{nM}$ pol $\beta$ at $37{ }^{\circ} \mathrm{C}$ for $15 \mathrm{~min}$. Reactions were terminated by transferring to $95{ }^{\circ} \mathrm{C}$ for $5 \mathrm{~min}$ in stopping buffer containing 95\% formamide and $10 \mathrm{mM}$ EDTA. Substrates and products were separated by $15 \%$ ureadenaturing PAGE and detected by a Pharos FX Plus Imager.

\subsection{Measurement of the efficiency of pol $\beta$ and APE1 activities in the context of a 3'- T/G mismatch}

The efficiency of pol $\beta$ and APE1 in extending or removing a 3'-terminus mismatched T was determined by measuring pol $\beta$ gap-filling synthesis activity and APE1 3'-5' exonuclease activity at various time intervals. Reactions were conducted by

incubating $25 \mathrm{nM} \mathrm{5}{ }^{\prime}-{ }^{32} \mathrm{P}$-labeled substrate with $5 \mathrm{nM}$ pol $\beta$ or increasing concentrations of APE1 alone at $5 \mathrm{nM}, 50 \mathrm{nM}$, and $100 \mathrm{nM}$. The reaction mixture was assembled on ice and incubated at $37^{\circ} \mathrm{C}$ for $1,2,5,10,15$, and 30 min intervals. Reactions were subsequently 
terminated by transferring to $95^{\circ} \mathrm{C}$ for $5 \mathrm{~min}$ in stopping buffer containing $95 \%$ formamide and $10 \mathrm{mM}$ EDTA. Substrates and products were separated by $15 \%$ urea-denaturing PAGE and detected by a Pharos FX Plus Imager.

\subsection{In vitro reconstituted BER assay}

The BER in the context of a T/G mismatch was reconstituted with purified APE1, pol $\beta$, FEN1, LIG I and the 1 nt-gapped substrate with a 3'-terminus matched $\mathrm{C} / \mathrm{G}$ or $\mathrm{T} / \mathrm{G}$ mismatch on the upstream primer, and a THF residue on the downstream primer opposite a template $\mathrm{C}$ or $\mathrm{T}$. Ten microliter reaction mixture contained BER buffer $(50 \mathrm{mM}$ Tris$\mathrm{HCl}, \mathrm{pH}$ 7.5, $50 \mathrm{mM} \mathrm{KCl}, 0.1 \mathrm{mM}$ EDTA, $0.1 \mathrm{mg} / \mathrm{ml} \mathrm{BSA}, 0.01 \%$ Nonidet P-40, $5 \mathrm{mM}$ $\left.\mathrm{MgCl}_{2}\right), 25 \mathrm{nM}$ substrate, $5 \mathrm{mM} \mathrm{Mg}{ }^{2+}, 50 \mu \mathrm{M}$ dNTPs, $2 \mathrm{mM}$ ATP and the indicated amounts of BER enzymes. The reaction mixture was assembled on ice and incubated at 37 ${ }^{\circ} \mathrm{C}$ for $15 \mathrm{~min}$. Reactions were terminated by transferring to $95^{\circ} \mathrm{C}$ for $5 \mathrm{~min}$ in stopping buffer containing $95 \%$ formamide and $10 \mathrm{mM}$ EDTA. Substrates and products were separated by $15 \%$ urea-denaturing PAGE and detected by a Pharos FX Plus Imager.

\subsection{Sequencing of repair products resulting from BER of an abasic lesion adjacent to}

\section{a T/G mismatch}

The BER of an abasic lesion adjacent to a $\mathrm{T} / \mathrm{G}$ mismatch was performed by incubating $50 \mathrm{nM}$ purified APE1, $5 \mathrm{nM}$ pol $\beta, 50 \mathrm{nM}$ FEN1, and $5 \mathrm{nM}$ LIG I with $25 \mathrm{nM}$ 1 nt-gapped substrate with a 3 '-terminus $\mathrm{T} / \mathrm{G}$ mismatch on the upstream primer, and a 5'THF residue on the downstream primer. The BER reaction $(20 \mu \mathrm{l})$ was reconstituted with the indicated concentrations of BER enzymes and substrates in BER reaction buffer (50 $\mathrm{mM}$ Tris- $\mathrm{HCl}, \mathrm{pH}$ 7.5, $50 \mathrm{mM} \mathrm{KCl}, 0.1 \mathrm{mM}$ EDTA, $0.1 \mathrm{mg} / \mathrm{ml} \mathrm{BSA}, 0.01 \%$ Nonidet P40, $5 \mathrm{mM} \mathrm{MgCl}_{2}$ ) that contained $50 \mu \mathrm{M}$ dNTPs, $5 \mathrm{mM} \mathrm{Mg}^{2+}$ and $2 \mathrm{mM}$ ATP. The reaction 
mixtures were assembled on ice, and incubated at $37^{\circ} \mathrm{C}$ for $15 \mathrm{~min}$. Reactions were then terminated by transferring to $95^{\circ} \mathrm{C}$ for $10 \mathrm{~min}$. To isolate the repair products, the template strand of the substrate was biotinylated at the 5'-end. The repair products were incubated with avidin agarose beads (Pierce-Thermo Scientific, Rockford, IL) in binding buffer that contained $0.1 \mathrm{M}$ phosphate, $0.15 \mathrm{M} \mathrm{NaCl}, \mathrm{pH} 7.2$ and $1 \%$ Nonidet $\mathrm{P}-40$ at $4^{\circ} \mathrm{C}$ for $2 \mathrm{~h}$ with rotation. The agarose beads were centrifuged at $5000 \mathrm{rpm}$ for $1 \mathrm{~min}$ and were washed three times with binding buffer. The repaired strands were then separated from their template strands through incubation in $0.15 \mathrm{M} \mathrm{NaOH}$ for 15 min with rotation under room temperature, followed by centrifugation at $5000 \mathrm{rpm}$ for $2 \mathrm{~min}$. The repaired strands were then precipitated with ethanol and dissolved in TE buffer. The repair products were then subjected to PCR amplification with a forward primer (5'-GCA GTC CTC TAG TCG TAG TAG-3') and a reverse primer (5'-GCA ATG AGT AAG TCT AGC TAC TAC-3'). The PCR amplification was performed under the following conditions: $95^{\circ} \mathrm{C}$ for $5 \mathrm{~min}, 1$ cycle; $95^{\circ} \mathrm{C}$ for $30 \mathrm{~s}, 55^{\circ} \mathrm{C}$ for $30 \mathrm{~s}$ and $72^{\circ} \mathrm{C}$ for $1 \mathrm{~min}, 35$ cycles; $72^{\circ} \mathrm{C}$ for $30 \mathrm{~min}$. The PCR products were then subjected to TA cloning using a TA cloning kit by following the manufacturer's instructions (Thermo Fisher Scientific, Pittsburgh, PA). White colonies were picked for amplifying plasmids that contained the inserts (repair products). Plasmids were isolated with a Miniprep plasmid isolation kit (Promega, Madison, WI), dissolved in TE buffer (10 mM Tris-HCl, pH 7.5, and $1 \mathrm{mM} \mathrm{EDTA),} \mathrm{and} \mathrm{subjected} \mathrm{to} \mathrm{DNA} \mathrm{sequencing.}$ Sequencing reactions were performed with the BigDye Terminator v3.1 Cycle Sequencing Kit (Thermo Fisher Scientific, Pittsburgh, PA) and were subjected to capillary electrophoresis (Florida International University DNA Sequencing Core Facility). Sequencing results were analyzed by MacVector 12.5.1 (MacVector, Apex, NC). 


\section{RESULTS}

\subsection{TDG is completely inhibited by an abasic lesion}

In $\mathrm{CpG}$ dinucleotide clusters, a $5 \mathrm{mC}$ can be converted to a $\mathrm{T}$ during active DNA demethylation, thereby resulting in a T/G mismatch (Morgan et al., 2004). On the other hand, guanines serve as hot spots of oxidative DNA damage, and guanines located in CpG dinucleotide clusters can be damaged leading to the production of a base lesion such as an abasic site adjacent to the $5 \mathrm{mC}$. However, it remains unknown whether an abasic lesion can affect TDG removal of a $\mathrm{T} / \mathrm{G}$ mismatch. To test this possibility, we initially examined the removal of the mismatched $\mathrm{T}$ from a $\mathrm{T} / \mathrm{G}$ mismatch adjacent to an abasic site by TDG. A substrate containing a mismatched $\mathrm{T} / \mathrm{G}$ adjacent to an abasic site analog, a THF residue, was used to mimic the intermediate with an abasic lesion generated after deamination of 5mC. This would allow TDG to remove the 3'-mismatched $\mathrm{T}$ generating a native abasic site, which was subsequently broken by a high temperature at $95^{\circ} \mathrm{C}$ into single-strand break intermediates as TDG products. The results showed that $50 \mathrm{nM}$ TDG removed the mismatched $\mathrm{T}$ next to a normal $\mathrm{G}$ (Figure 2.1, lane 2). Its activity was significantly increased with increasing concentrations of TDG from $60 \mathrm{nM}$ to $70 \mathrm{nM}$ (Figure 2.1, lanes 3-4). Surprisingly, the same concentrations of TDG (50 to $70 \mathrm{nM}$ ) failed to remove a mismatched T next to an abasic site (Figure 2.1, lanes 6-8). This indicated that TDG activity was completely inhibited by an abasic lesion adjacent to a $\mathrm{T} / \mathrm{G}$ mismatch. This further suggests that the presence of an abasic lesion next to an intermediate of $5 \mathrm{mC}$ demethylation in $\mathrm{CpG}$ dinucleotide clusters can lead to accumulation of $\mathrm{T} / \mathrm{G}$ mismatches. 

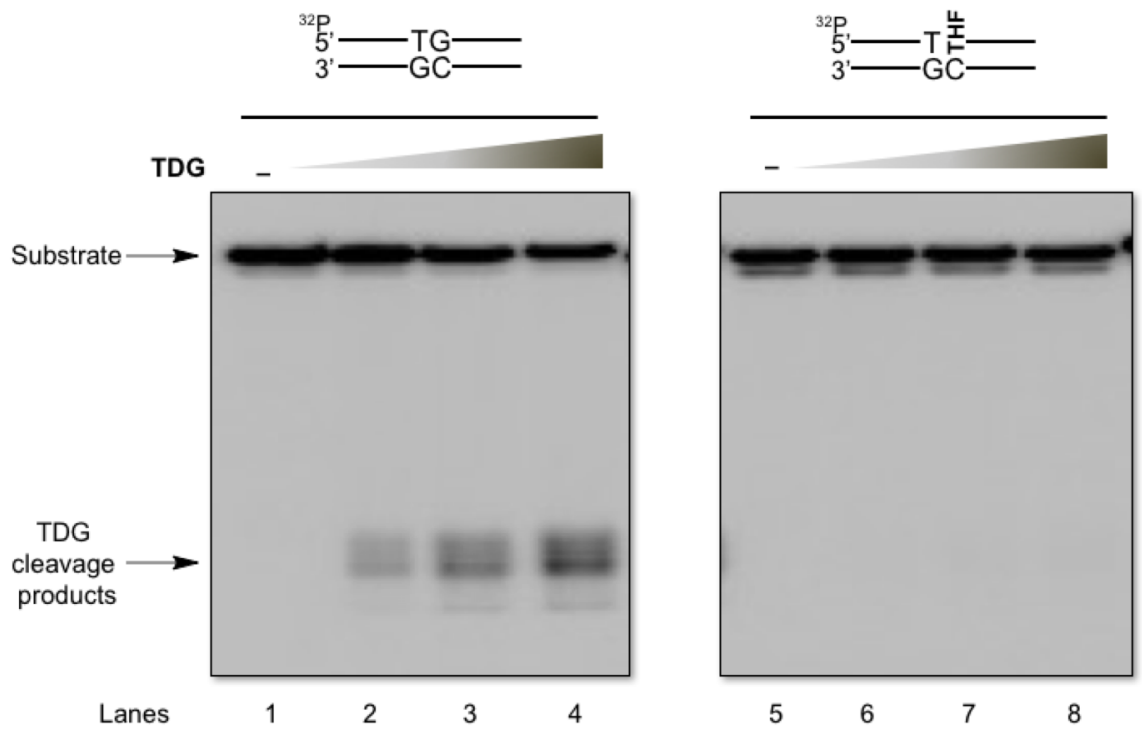

Figure 2.1 The activity of TDG in removing a T/G mismatch at a $\mathrm{CpG}$ dinucleotide is inhibited by an adjacent abasic lesion. An oligonucleotide substrate that contained random DNA sequence with a THF residue next to a $5^{\prime}-\mathrm{T} / \mathrm{G}$ mismatch in a $\mathrm{CpG}$ dinucleotide was used to mimic a BER intermediate generated from removal of 8-oxoGby OGG1 and deamination of 5mC. The substrate that contained a $\mathrm{G}$ with a $5^{\prime}-\mathrm{T} / \mathrm{G}$ mismatch was employed to mimic an intermediate generated by deamination of $5 \mathrm{mC}$ alone. TDG activity in removing a $\mathrm{T} / \mathrm{G}$ mismatch was measured as described in "Materials and methods". Substrates were ${ }^{32} \mathrm{P}$-labeled at the 5 '-end of the damaged strand. Lanes 1 and 5 correspond to the substrate only. Lanes 2 and 6 correspond to reaction mixtures with $50 \mathrm{nM}$ TDG. Lanes 3 and 7 correspond to reaction mixtures with $60 \mathrm{nM}$ TDG. Lanes 4 and 8 correspond to reaction mixtures with $70 \mathrm{nM}$ TDG.TDG cleavage products are indicated. Substrates are illustrated schematically above the gel.

\subsection{APE1 can efficiently incise an abasic site that is adjacent to a $T / G$ mismatch in the}

\section{presence of TDG}

To further determine if a 3'-mismatched T affects APE1 activity, APE1 5'-incision of an abasic site adjacent to a $\mathrm{T} / \mathrm{G}$ mismatch was examined in the absence and presence of TDG. Substrates containing a 3'-mismatched T next to a THF residue opposite to a C or a $\mathrm{T}$ were used for measuring APE1 activity. The substrates mimic the intermediates 
generated by deamination of the $5 \mathrm{mC}$ on the damaged strand alone or the $5 \mathrm{mCs}$ on both the damaged strand and template strand simultaneously. The results showed that $5 \mathrm{nM}$ APE1 efficiently carried out 5'-incision of an abasic site in the absence and presence of TDG (Figure 2.2, lanes 2-6 and lanes 8-12).

\subsection{Pol $\beta$ can efficiently extend the 3'-mismatched $T$ of a $T / G$ mismatched base pair}

Since APE1 5'-incision of an abasic site next to a T/G mismatch resulted in the 1nt gap intermediate with a 3'-mismateched T, which would be subsequently passed to pol $\beta$ for continuation of repair of the lesion, we then asked if pol $\beta$ could still perform DNA synthesis in the presence of a 3'-mismatched T. To test this, we examined pol $\beta$ gap-filling synthesis using the substrates with a 3'-matched $\mathrm{C}$ or a 3'-mismatched $\mathrm{T}$ adjacent to a preincised THF residue or a 3'-T along with a template $\mathrm{T}$ opposite a preincised THF residue. We found that $1 \mathrm{nM}, 5 \mathrm{nM}$ and $10 \mathrm{nM}$ pol $\beta$ efficiently extended the 3'-matched C (Figure 2.3, lanes 2.4). However, surprisingly, pol $\beta$ also efficiently extended a 3'-mismatched $T$ (Figure 2-3, lanes 6-8 and lanes 10-12), particularly at the concentrations of $5 \mathrm{nM}$ and 10 $\mathrm{nM}$ (Figure 2-3, lanes 7-8 and 11-12). For all substrates, pol $\beta$ mainly inserted one nucleotide to extend the 3'-terminus $\mathrm{T}$ and fill in the gap. Interestingly, pol $\beta$ extension of a 3'-mismatched $\mathrm{T}$ with a template $\mathrm{T}$ was much weaker than its synthesis with a template $\mathrm{C}$, indicating that the polymerase had difficulty in extending a 3'-mismatched $\mathrm{T}$ when it incorporated A to base pair with a template T. The results further suggest that pol $\beta$ can tolerate a 3'-terminus mismatched nucleotide, similar to DNA translesion synthesis polymerases such as pol $\kappa$ and pol $\mu$ that exhibit mismatch tolerance (Wolfle et al., 2003; Zhang et al., 2001). Moreover, pol $\beta$ extension of a 3'-terminus matched C, but not 3'mismatched $\mathrm{T}$ resulted in small amount of products with more than one nucleotide insertion 
(Figure 2-3, lanes 3-5). This indicates that pol $\beta$ performed strand-displacement synthesis to extend a 3'-matched C, however it failed to perform this synthesis when it extended a 3'-mismatched $\mathrm{T}$ indicating that the mismatched base inhibited further pol $\beta$ DNA synthesis. In conclusion, pol $\beta$ tolerated and efficiently extended a 3'-mismatched $\mathrm{T}$ resulting from deamination of a $5 \mathrm{mC}$ in $\mathrm{CpG}$ dinucleotides during BER.

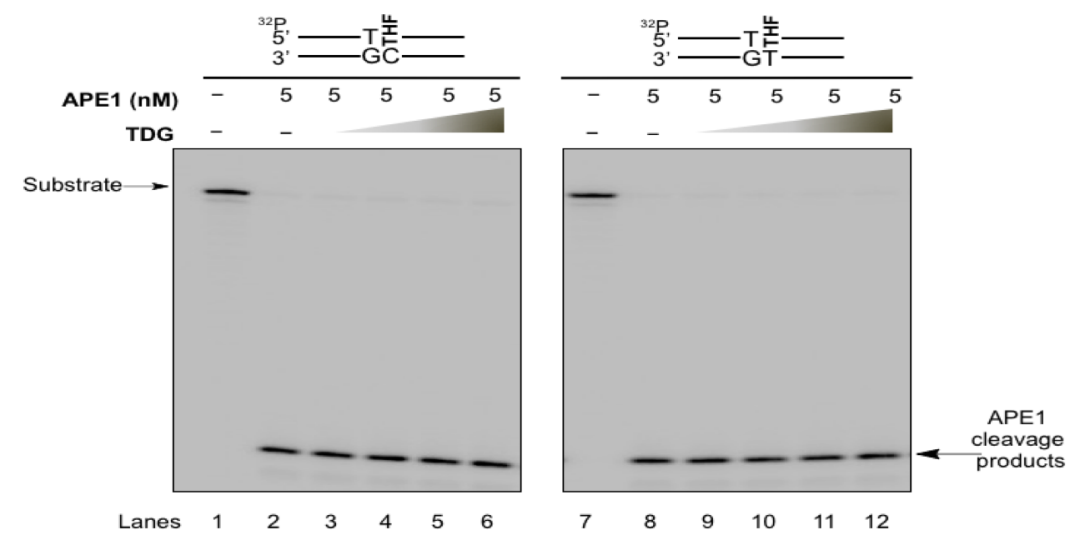

Figure 2.2 APE1 can efficiently incise an abasic site that is adjacent to a T/G mismatch in a CpG dinucleotide in the presence of TDG. APE1 5'-incision of an abasic site that is adjacent to a 5'-T/G mismatch in a $\mathrm{CpG}$ dinucleotide was examined under the conditions described in "Materials and methods". Substrates were ${ }^{32} \mathrm{P}$-labeled at the 5 '-end of the damaged strand. Lanes 1 and 7 correspond to the substrate only. Lanes 2 and 8 correspond to reaction mixtures with 5 $\mathrm{nM}$ APE1 alone. Lanes 3 and 9 correspond to reaction mixtures with $5 \mathrm{nM}$ APE1 in the presence of $10 \mathrm{nM}$ TDG. Lanes 4 and 10 correspond to reaction mixtures with $5 \mathrm{nM}$ APE1 in the presence of $25 \mathrm{nM}$ TDG. Lanes 5 and 11 correspond to reaction mixtures with $5 \mathrm{nM}$ APE1 in the presence of $50 \mathrm{nM}$ TDG. Lanes 6 and 12 correspond to reaction mixtures with $5 \mathrm{nM}$ APE1 in the presence of $75 \mathrm{nM}$ TDG. Substrates and APE1 cleavage products are indicated. The substrates are illustrated schematically above the gel. 


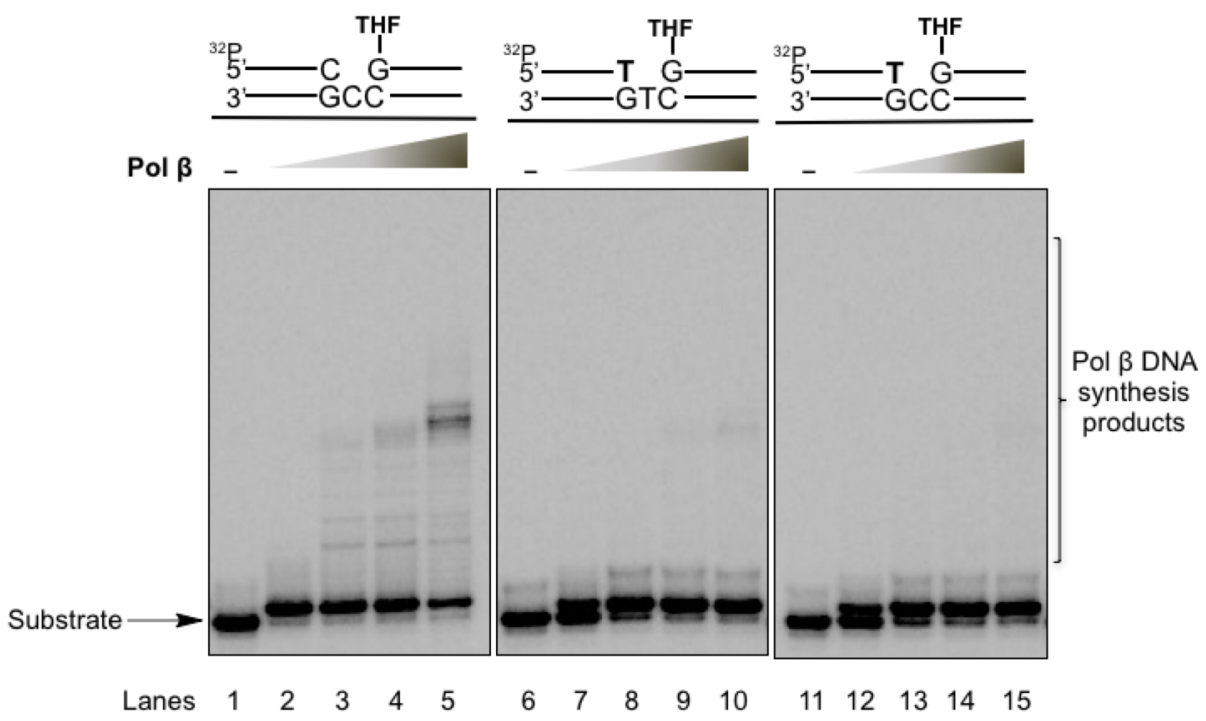

Figure 2.3 Pol $\beta$ extends a 3'-terminus mismatched $\mathbf{T}$ at a $\mathbf{C p G}$ dinucleotide. Pol $\beta$ DNA synthesis in the context of a $\mathrm{T} / \mathrm{G}$ mismatch was examined as described in "Materials and methods". Substrates were designed to mimic a BER intermediate containing a 3'-terminus matched $\mathrm{C}$, or 3'-terminus mismatched $\mathrm{T}$ in a $\mathrm{CpG}$ dinucleotide with an abasic site preincised by APE1 opposite to a $\mathrm{C}$ or $\mathrm{T}$ on the template strand. Substrates were ${ }^{32} \mathrm{P}$-labeled at the 5 '-end of the damaged strand. Lanes 1, 5, and 9 correspond to the substrate only. Lanes 2, 6, and 10 correspond to reaction mixtures with $1 \mathrm{nM}$ pol $\beta$. Lanes 3, 7, and 11 correspond to reaction mixtures with $5 \mathrm{nM}$ pol $\beta$. Lanes 4,8 , and 12 represent reaction mixtures with $10 \mathrm{nM}$ pol $\beta$. Substrates and pol $\beta$ DNA synthesis products are indicated. Substrates are illustrated schematically above the gel.

\subsection{APE1 3'-5' exonulcease activity efficiently removes a 3'-mismatched T}

It has been reported that APE1 3'-5' exonuclease activity can remove mismatched nucleotides (Chou and Cheng, 2002; Cistulli et al., 2004; Liu et al., 2007b). Thus, we further hypothesized that APE1 might also remove the 3'-mismatched $T$ from a $T / G$ mismatch to increase the fidelity of pol $\beta$ in a $\mathrm{CpG}$ dinucleotide. To test the hypothesis, we examined APE1 3'-5' exonuclease activity on the substrate (25 nM) with a 3'-matched C or 3'-mismatched $\mathrm{T}$ on the upstream strand and a 5'-THF residue on the downstream strand 
with a template $\mathrm{C}$ or $\mathrm{T}$ opposite an abasic site. We found that APE1 ( $5 \mathrm{nM}, 10 \mathrm{nM}, 25 \mathrm{nM}$ and $50 \mathrm{nM}$ ) exhibited poor cleavage activity to remove the 3'-matched C (Figure 2.4, lanes 2-5), indicating inefficient removal of a 3'-matched nucleotide by APE1 3'-5' exonuclease activity. However, APE1 removed the 3'-mismatched T much more efficiently than it removed a 3'-matched C (Figure 2.4, lanes 12-15 and 7-10). For all the substrates with a T/G mismatch, APE1 mainly removed one nucleotide from the upstream primer containing a 3'-mismatched T. Thus, APE1 3'-5' exonuclease efficiently removed the 3'-mismatched T. Interestingly, we found that APE1 3'-5' exonuclease exhibited much weaker activity in removing the 3'-mismatched $\mathrm{T}$ located next to a template $\mathrm{T}$ than in removing the mismatched nucleotide next to a $\mathrm{C}$ on the template strand (Figure 2.4, lanes 7-10), indicating that simultaneous demethylation of $5 \mathrm{mCs}$ on both the damaged and template strands also decreased APE1 3'-5' exonuclease activity.

\subsection{FEN1 cleaves a sugar phosphate flap adjacent to a 3'-T/G mismatch}

To further determine whether a 3'-terminus mismatched $\mathrm{T}$ will have any effect on FEN1 cleavage, we characterized FEN1 cleavage activity with the substrates $(25 \mathrm{nM})$ containing an upstream 3'-C or 3'-T and a downstream 5'-THF residue and a template C or $\mathrm{T}$ in the absence and presence of pol $\beta$. We found that in the absence of pol $\beta$, FEN1 (5 $\mathrm{nM}, 10 \mathrm{nM}$ and $25 \mathrm{nM}$ ) exhibited poor cleavage activity in removing the 5'-THF flap (Figure 2.5, lanes 2-4, 9-11 and 16-18), indicating inefficient removal of a 5'-THF flap adjacent to a one-nucleotide gap by FEN1. In the presence of pol $\beta$ DNA synthesis, FEN1 cleavage of the substrate with a 3'-matched C resulted in an increased amount of smaller products resulting from FEN1 cleavage of a longer flap (Figure 2.5, lanes 5-7), indicating that pol $\beta$ strand-displacement synthesis created a longer flap for FEN1 cleavage. 
However, the presence of pol $\beta$ DNA synthesis did not significantly improve FEN1 flap cleavage on the substrate with a 3'-mismatched T (Figure 2.5, lanes 12-14 and 19-21). This indicated that FEN1 cleavage of a flap next to a 3'-mismatched T was inhibited even in the presence of pol $\beta$. This appeared to result from the inhibition of pol $\beta$ stranddisplacement synthesis by a 3'-T/G mismatch (Figure 2.3).

\subsection{The efficiency of pol $\beta$ extension of a 3'-mismatched T and APE1 3'-5' exonuclease to remove the 3'-mismatched T}

Since our previous results indicate that removal of a 3'-mismatched T by APE1 is critical for improving pol $\beta$ fidelity, we then asked if APE1 could remove a 3'-mismatch T before pol $\beta$ could extend the mismatched nucleotide during BER. We tested this by examining the efficiency of pol $\beta$ DNA synthesis at $5 \mathrm{nM}$ and that of APE1 3'-5' exonuclease activity at $5 \mathrm{nM}, 50 \mathrm{nM}$ and $100 \mathrm{nM}$ using the substrate containing a 3 'mismatched $\mathrm{T}$ and a 5'-THF residue with a template $\mathrm{C}$ opposite the THF residue. The enzymatic reaction products were measured at different time intervals ranging from $0 \mathrm{~min}$ to $30 \mathrm{~min}$. The percentage of products was plotted against time (Figure 2.6). The results revealed that $5 \mathrm{nM}$ pol $\beta$ extended a 3'-mismatched T much more efficiently than APE1 3'5' exonuclease removed the mismatched nucleotide at the same concentration. However, the $3^{\prime}-5$ ' exonuclease activity of APE1 at $50 \mathrm{nM}$ and $100 \mathrm{nM}$ removed the mismatched T

much more efficiently than $5 \mathrm{nM}$ pol $\beta$ extended the mismatched nucleotide (Figure 2.6). This indicated that a high concentration of APE1 was sufficient to combat pol $\beta$ extension of a mismatched nucleotide. Since APE1 is much more abundant than pol $\beta$ in mammalian cells, the results further suggest that APE1 can remove a mismatched $\mathrm{T}$ before it is extended by pol $\beta$ in cells. 


\subsection{BER can occur in the presence of 3 '-T/G mismatch}

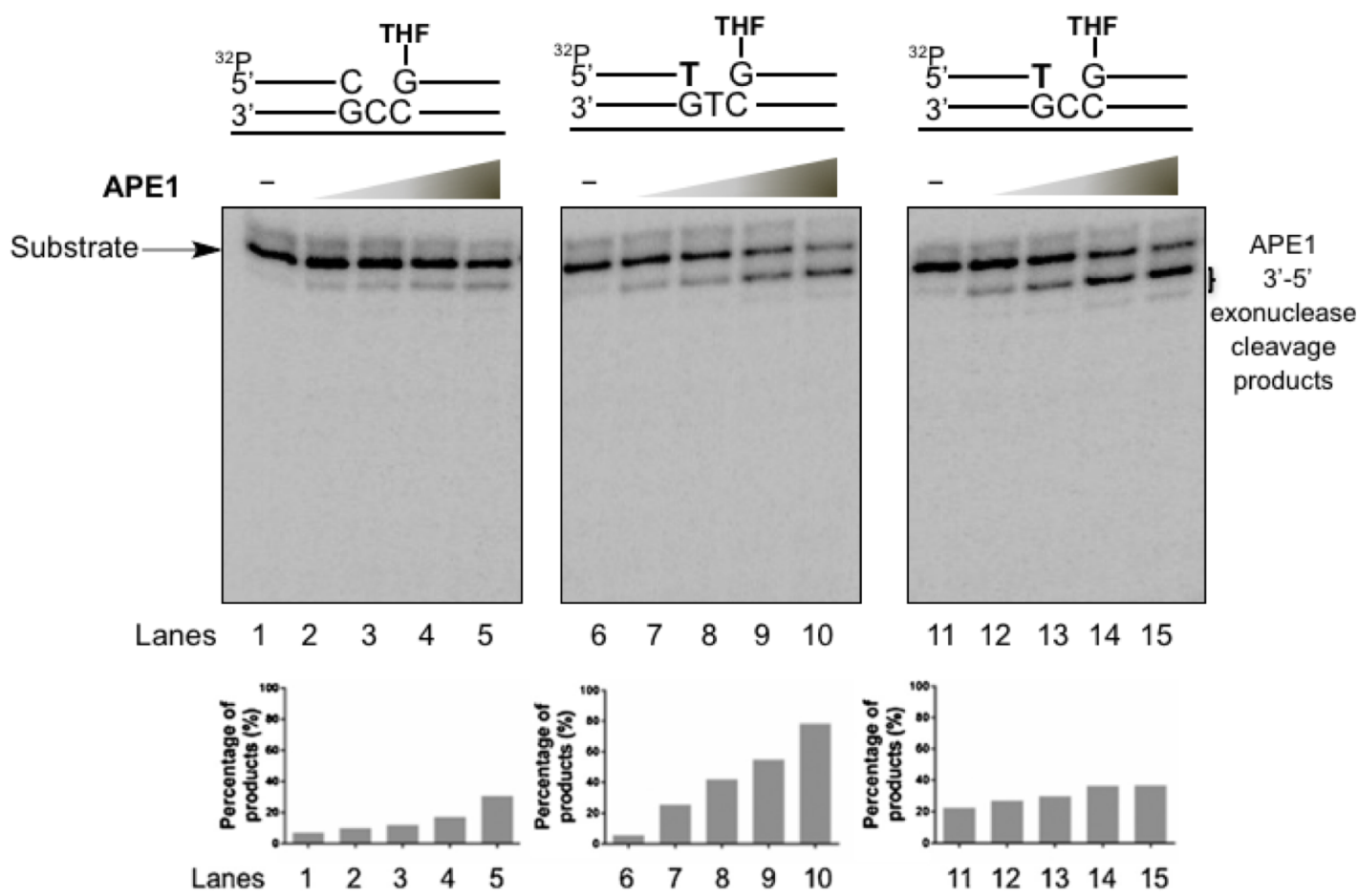

Figure 2.4 APE1 3'-5' exonuclease activity removes a 3 '-terminus mismatched $T$ in a CpG dinucleotide. APE1 3'-5' exonuclease activity in removing a 3'-mismatched $\mathrm{T}$ in a CpG dinucleotide was measured as described in "Materials and methods". Substrates were ${ }^{32} \mathrm{P}$-labeled at the 5 '-end of the damaged strand. Lanes 1, 6 and 11 correspond to the substrate only. Lanes 2, 7 and 12 correspond to reaction mixtures with $5 \mathrm{nM}$ APE1. Lanes 3, 8 and 13 correspond to reaction mixtures with $10 \mathrm{nM}$ APE1. Lanes 4, 9 and 14 correspond to reaction mixtures with $25 \mathrm{nM}$ APE1. Lanes 5, 10 and 15 correspond to reaction mixtures with $50 \mathrm{nM}$ APE1. Substrates and APE1 cleavage products are indicated. Substrates are illustrated schematically above the gel. The quantification of the APE1 3' $-5^{\prime}$ exonuclease cleavage products are shown below the gels.

To examine whether a 3 , $\mathrm{T} / \mathrm{G}$ mismatch can ultimately result in the production of BER products, we reconstituted BER by incubating the substrates without or with a $T / G$ mismatch with APE1, pol $\beta$, FEN1 and LIG I. We found that a significant amount of repair products were produced during BER with a 3'-mismatched $\mathrm{T}$ in the absence of APE1 
(Figure 2.7A, lanes 6 and 10) although the amount of products was less than those formed

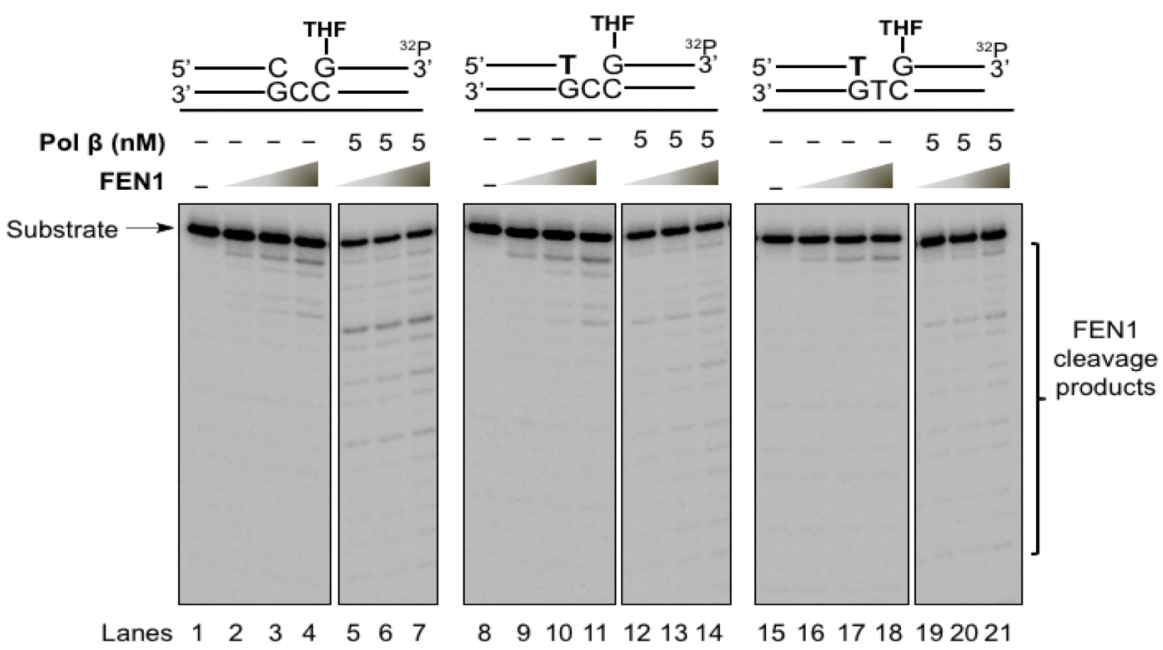

Figure 2.5 FEN1 flap cleavage in the presence of a T/G mismatch in a $\mathrm{CpG}$ dinucleotide. FEN1 flap cleavage activity in the presence of a $\mathrm{T} / \mathrm{G}$ mismatch in a $\mathrm{CpG}$ dinucleotide was examined as described in "Materials and methods". Substrates were ${ }^{32} \mathrm{P}$-labeled at the 3 '-end of the damaged strand. Lanes 1, 8 and 15 correspond to the substrate only. Lanes 2, 9 and 16 correspond to reaction mixtures with 5 nM FEN1. Lanes 3, 10 and 17 correspond to reaction mixtures with $10 \mathrm{nM}$ FEN1. Lanes 4, 11 and18 correspond to reaction mixtures with $25 \mathrm{nM}$ FEN1. Lanes 5, 12 and 19 correspond to reaction mixtures that contained $5 \mathrm{nM}$ FEN1 in the presence of $5 \mathrm{nM}$ pol $\beta$. Lanes 6,13 and 20 represent reaction mixtures that contained $10 \mathrm{nM}$ FEN1 in the presence of $5 \mathrm{nM}$ pol $\beta$. Lanes 7, 14 and 21 represent reaction mixtures that contained $25 \mathrm{nM}$ FEN1 in the presence of $5 \mathrm{nM}$ pol $\beta$. Substrates and FEN1 cleavage products are indicated. Substrates are illustrated schematically above the gel.

from the substrate with a 3'-matched C (Figure 2.7A, lane 2). This indicated that in the absence of APE1, pol $\beta$ extended a 3'-mismatched T resulting in repair product with a T/G mismatch. We found that presence of high concentrations of APE1 (50 nM and $100 \mathrm{nM})$ significantly stimulated the production of repair products (Figure 2.7A, lanes 7-8) during BER with the substrate containing a 3'-mismatched $\mathrm{T}$ and a template $\mathrm{C}$. However, this effect was not evident with the substrate containing a 3'-mismatched $\mathrm{T}$ and a template $\mathrm{T}$ (Figure 2.7A, lanes 11-12). This indicated that BER was accomplished through pol $\beta$ 
tolerance of a 3'-mismatched $\mathrm{T}$ suggesting that this can further result in a $\mathrm{C}$ to $\mathrm{T}$ mutation, and APE1 may reduce the production of the mutation.

\subsection{APE1 significantly reduces $C$ to $T$ mutations resulting from a $T / G$ mismatch during BER}

To further confirm whether APE1 can reduce mutations resulting from extension of a 3'-mismatched T by pol $\beta$ during BER, we sequenced the repair products generated from reconstituted BER with the substrates containing a 3'-mismatched $\mathrm{T}$ adjacent to a THF residue opposite a template $\mathrm{C}$ or $\mathrm{T}$ in the absence and presence of APE1. The results showed that in the absence of APE1, all repair products $(100 \%)$ contained a mismatched T (Figure 2.7B). This indicated that the mismatched $\mathrm{T}$ was fully extended by pol $\beta$ during BER. However, in the presence of $50 \mathrm{nM}$ APE1, only $7.7 \%-13 \%$ of the repair products contained a mismatched $\mathrm{T}$, and $87 \%-92.3 \%$ of the mismatched Ts were converted into matched Cs (Figure 2.7B). This indicated that APE1 3'-5' exonuclease activity efficiently removed mismatched Ts allowing pol $\beta$ to insert a $\mathrm{C}$ to base pair with a template $\mathrm{G}$ during BER. Taken together, our results indicated that APE1 3'-5' exonuclease significantly reduced $\mathrm{T} / \mathrm{G}$ mismatches, thereby preventing mutations in $\mathrm{CpG}$ dinucleotides during BER. 


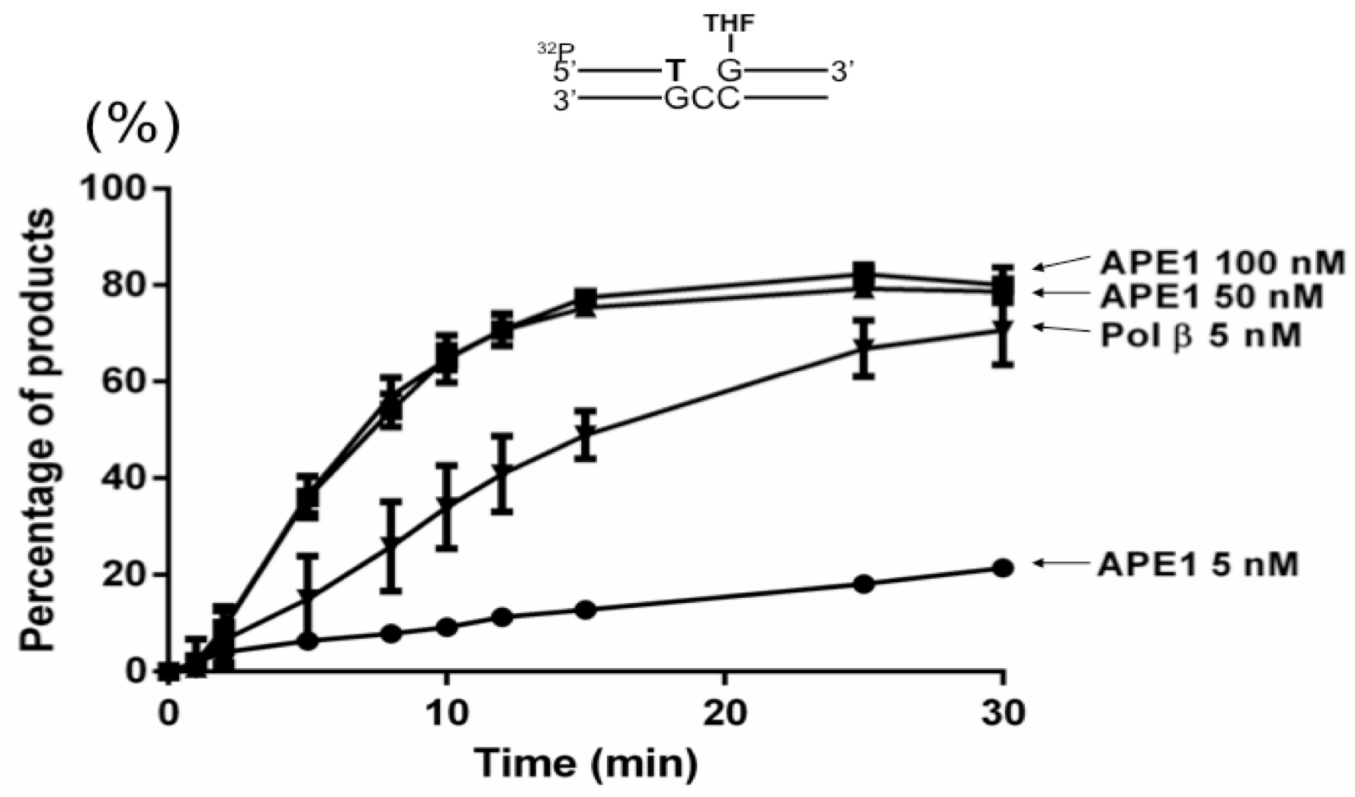

Figure 2.6 The activities of pol $\beta$ DNA synthesis and APE1 3'-5' exonuclease in the context of a T/G mismatch in a CpG dinucleotide. The activities of pol $\beta$ and APE1 in extending or removing a 3'-terminus mismatched $\mathrm{T}$ were determined at various time intervals $(1,2,5,10,15$ and $30 \mathrm{~min}$ ) with a substrate containing a 3 '-terminus mismatched $\mathrm{T}$ in a $\mathrm{CpG}$ dinucleotide with a THF residue preincised by APE1 as described in "Materials and methods". The amount of products resulting from pol $\beta$ DNA synthesis or APE1 3' -5 ' exonuclease activity were quantified by using Quantity One Software (Bio-Rad Laboratories, Hercules, CA). The percentage of products was calculated as the amount of products over total amount of substrates and was plotted against time.

\section{DISCUSSION}

In this study, for the first time, we explored the role of BER in maintaining the integrity of $\mathrm{CpG}$ islands by removing a mismatched base when DNA base damage occurs next to a $\mathrm{T} / \mathrm{G}$ mismatch resulting from deamination of a $5 \mathrm{mC}$ in a $\mathrm{CpG}$ dinucleotide. Since CpGs are susceptible to oxidative DNA damage, a DNA base lesion such as 8-oxoG or an abasic site can be readily generated next to a $\mathrm{T} / \mathrm{G}$ mismatch. In this scenario, both the mismatched $\mathrm{T}$ and base lesion need to be resolved by the BER pathway. Thus an important 


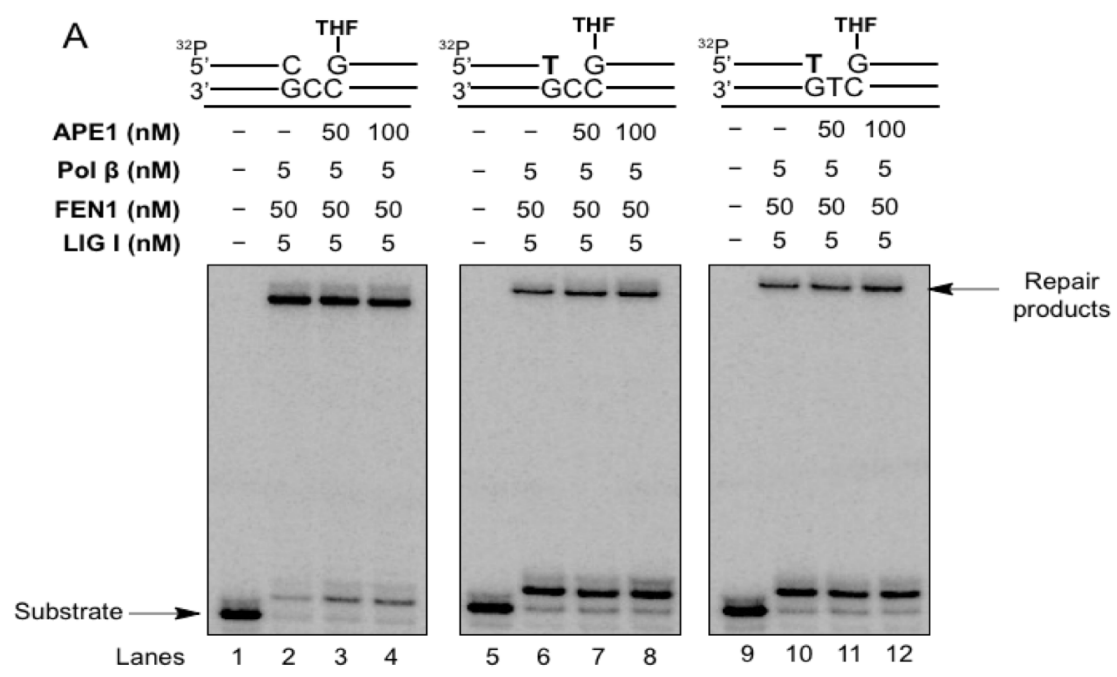

B

DNA sequencing results of repair products

\begin{tabular}{|c|c|c|}
\hline Substrate & $\begin{array}{l}\text { Percentage of } \\
\text { mismatched } T\end{array}$ & $\begin{array}{l}\text { Percentage of } \\
\text { matched C }\end{array}$ \\
\hline \multicolumn{3}{|c|}{ BER reconstitution without APE1 } \\
\hline $5^{\prime}=\frac{\text { THF }}{\mathrm{T}}=$ & $100 \%$ & $0 \%$ \\
\hline 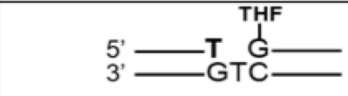 & $100 \%$ & $0 \%$ \\
\hline \multicolumn{3}{|c|}{ BER reconstitution with APE1 } \\
\hline $5^{\prime}={ }^{\text {THF }} \stackrel{\text { G- }}{\mathrm{G}}=$ & $7.7 \%$ & $92.3 \%$ \\
\hline $\begin{array}{c}\text { THF } \\
5^{\prime}-\mathbf{T} \stackrel{+}{\mathrm{G}}-\mathrm{GTC}-\end{array}$ & $13.0 \%$ & $87.0 \%$ \\
\hline
\end{tabular}

Figure 2.7 BER reconstitution in the context of a $\mathrm{T} / \mathrm{G}$ mismatch in a $\mathrm{CpG}$ dinucleotide. (A) BER reconstitution in the context of a $\mathrm{T} / \mathrm{G}$ mismatch was performed as described in "Materials and Methods". Substrates, were ${ }^{32} \mathrm{P}$-labeled at the 5'-end of the damaged strand. Lanes 1, 5 and 9 correspond to substrate only. Lanes 2, 6 and 10 correspond to reaction mixtures that contained $5 \mathrm{nM}$ pol $\beta, 50 \mathrm{nM}$ FEN1 and $5 \mathrm{nM}$ LIG I. Lanes 3, 7 and 11 correspond to reaction mixtures that contained $50 \mathrm{nM}$ APE1, $5 \mathrm{nM}$ pol $\beta, 50 \mathrm{nM}$ FEN1 and 5 nM LIG I. Lanes 4, 8 and 12 correspond to reaction mixtures that contained $100 \mathrm{nM} \mathrm{APE} 1,5$ $\mathrm{nM}$ pol $\beta, 50 \mathrm{nM}$ FEN1 and $5 \mathrm{nM}$ LIGI. Substrates and repair products are indicated. Substrates are illustrated schematically above the gel. (B) DNA sequencing results of repair products resulting from BER of a $\mathrm{T} / \mathrm{G}$ mismatch and abasic lesion in a $\mathrm{CpG}$ dinucleotide. Repair products were separated from the template strand with avidin beads. Subsequently, repair products were amplified by PCR and cloned into a TA vector and subjected to DNA sequencing. The percentage of repair products that contained a mismatched $\mathrm{T}$ or matched $\mathrm{C}$ from the different substrates are shown.

issue is how BER, in the context of the two different types of base lesions, may be initiated. 
It has been shown that OGG1 removes an 8-oxoG next to a 5'-T/G mismatch about 3.5fold faster than TDG removes the mismatched T (Sassa et al., 2014). This indicates that the 8-oxoG is usually removed by OGG1 prior to the removal of the mismatched T by TDG. This results in an abasic site next to the $5^{\prime}-\mathrm{T} / \mathrm{G}$ mismatch. Surprisingly, we found that the activity of TDG of removing the mismatched $\mathrm{T}$ adjacent to an abasic site was completely inhibited by the abasic lesion (Figure 2.1, lanes 6-8) indicating that an abasic lesion next to the mismatched nucleotide completely suppressed the removal of the mismatched $\mathrm{T}$ by TDG. However, we showed that APE1 incised an abasic lesion efficiently in the presence of the mismatched $\mathrm{T}$ (Figure 2.2, lanes 3-6 and lanes 9-12) leading to the production of a single-strand break intermediate with the 3'-terminus mismatched $\mathrm{T}$. We found that pol $\beta$ efficiently extended the 3'-mismatched $\mathrm{T}$ (Figure 2.3, lanes 6-8 and lanes 10-12), thereby resulting in maintenance of the mismatched base in the repair products (Figure 2.7A, lanes 6-8 and lanes 10-12). This subsequently led to a $\mathrm{C}$ to $\mathrm{T}$ transition mutation in the repair products (Figure 2.7B). However, the pol $\beta$-mediated mutagenic effect was significantly reduced (Figure 2.7B) through the removal of the 3'-mismatched T by APE1 3'-5' exonuclease activity (Figure 2.4, lanes 7-10 and lanes 12-15 and Figure 6). Our results support a model whereby a base lesion such as an abasic lesion occurs adjacent to a T/G mismatched basepair in a $\mathrm{CpG}$ dinucleotide during DNA demethylation. Removal of the mismatched T by TDG is completely inhibited by the abasic lesion. This allows APE1 to incise the abasic site at its 5'-end to initiate BER. This subsequently 


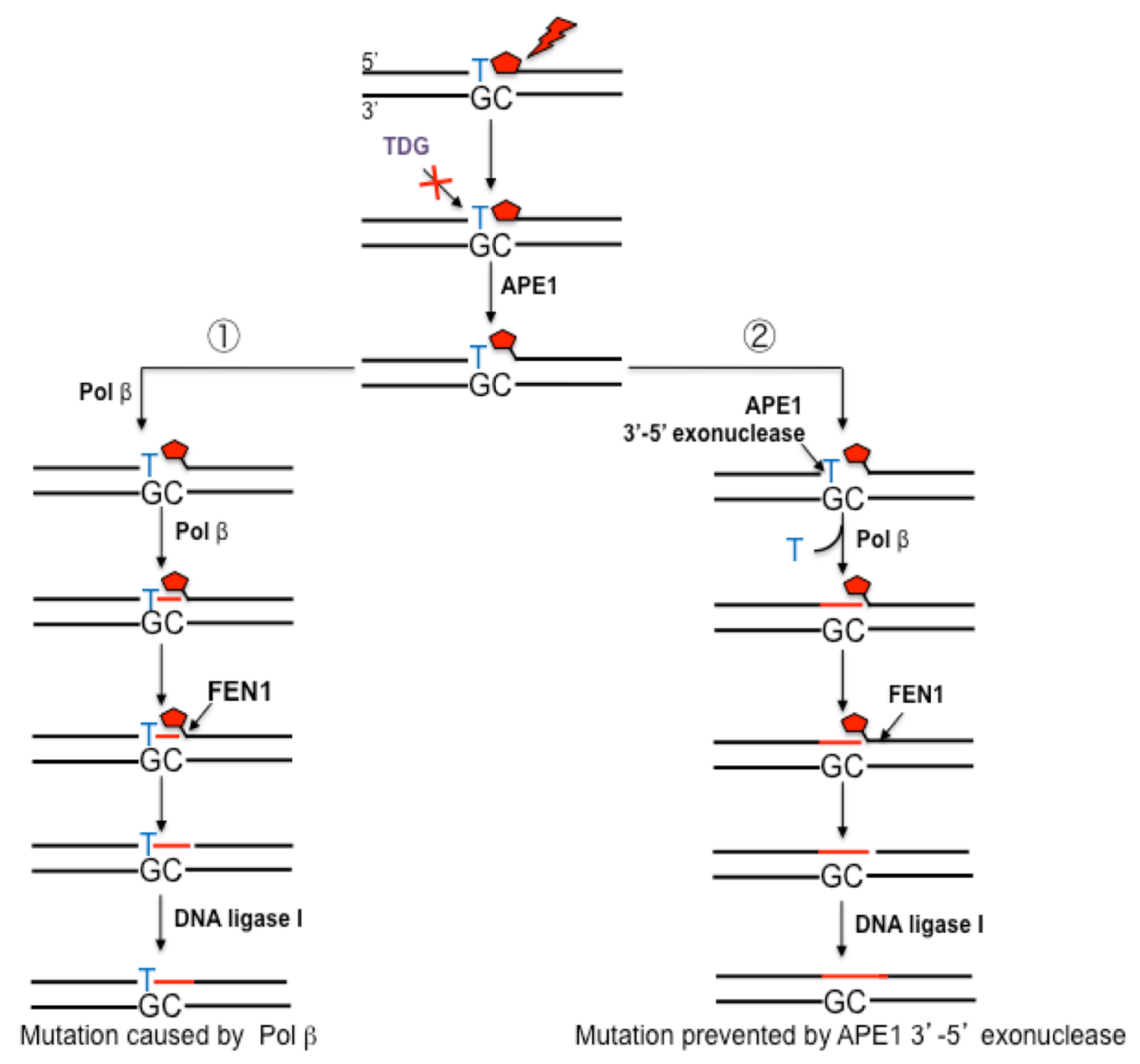

Figure 2.8 APE1 3'-5' exonuclease combats the extension of a $T / G$ mismatch by pol $\beta$ and $\mathbf{C}$ to $\mathbf{T}$ mutations in CpGs during BER. During active DNA demethylation of $5 \mathrm{mC}$ in $\mathrm{CpG}$ dinucleotides, the $5 \mathrm{mC}$ is deaminated to $\mathrm{T}$ thereby leading to production of a $\mathrm{T} / \mathrm{G}$ mismatch, while the adjacent $\mathrm{G}$ can also be damaged by oxidative stress resulting in an abasic lesion. TDG activity in removing the $\mathrm{T} / \mathrm{G}$ mismatch is completely inhibited by the abasic lesion. This allows the accumulation of $\mathrm{T} / \mathrm{G}$ mismatches that are adjacent to abasic lesions. APE1 can efficiently incise the resulting abasic site, leading to a 1 nt gap with a 3'-terminus mismatched T. Pol $\beta$ can extend the mismatched $\mathrm{T}$ to create a short flap with a sugar phosphate. Subsequently, FEN1 removes the flap, generating a nick for ligation by DNA ligase I. This leads to a $\mathrm{C}$ to $\mathrm{T}$ transition mutation (Sub-pathway 1). In a scenario where the 3 '-terminus mismatched $T$ is efficiently removed by APE1 3' -5 ' exonuclease prior to pol $\beta$ extension of the mismatch nucleotide, pol $\beta$ incorporates a $\mathrm{C}$ to base pair with a template $\mathrm{G}$. This prevents a $\mathrm{C}$ to $\mathrm{T}$ mutation (Sub-pathway 2).

leads to a 1nt gapped DNA with a 3'-mismatched T that can be subjected to two different subpathways (Figure 2.8). If the 3'-mismatched T is captured by pol $\beta$ before it is removed 
by APE1 3' -5 ' exonuclease activity, it is extended by pol $\beta$. This then results in the sustainment of the 3'-mismatched $\mathrm{T}$ during BER, thereby causing a $\mathrm{C}$ to $\mathrm{T}$ transition mutation (Figure 2.8, subpathway 1). However, if the 3'-mismatched $\mathrm{T}$ is removed by APE1 3'-5' exonuclease activity before it is extended by pol $\beta$, pol $\beta$ fills in the gap by incorporating a correct nucleotide to accomplish BER (Figure 2.8, subpathway 2). In this scenario, APE1 3'-5' exonuclease activity prevents mutation during BER in the context of a T/G mismtach DNA demethylation intermediate.

Here, we provided the first evidence that APE1 3'-5' exonuclease can prevent $\mathrm{C}$ to $\mathrm{T}$ mutations during BER of an abasic lesion adjacent to a $\mathrm{T} / \mathrm{G}$ mismatch in a $\mathrm{CpG}$ dinucleotide, thereby maintaining the integrity of CpGs. APE1 3'-5' exonuclease activity was initially reported to be able to remove a 3'-mismatched nucleotide in DNA (Chou and Cheng, 2002). It has been found that the efficiency of the 3'-5' exonuclease in removing a mismatched nucleotide is 160 -fold higher than that in removing a matched nucleotide (Chou and Cheng, 2002). Thus, it has been proposed that APE1 3'-5' exonuclease serves as a proofreading enzyme for correcting mismatched nucleotides incorporated by pol $\beta$. This is further suggested by a previous finding showing that APE1 forms a complex with pol $\beta$ on a nicked or $1 \mathrm{nt}$ gapped DNA with a deoxyribonucleotide phosphate (dRP) flap (Liu et al., 2007b). Although the APE1-pol $\beta$-DNA ternary complex failed to significantly stimulate APE1 3'-5' exonuclease activity on the nicked DNA (Liu et al., 2007b), it is possible that the complex may facilitate APE1 3'-5' exonuclease activity at a gapped DNA with a sugar phosphate. Moreover, our results showed that high concentrations of APE1 removed the 3'-mismatched T more efficiently than pol $\beta$ extension of the 3'-mismatched nucleotide. Thus, it is conceivable that a relatively high concentration of APE1 in 
mammalian cells exhibits a robust 3 ' -5 ' exonuclease activity that can cooperate with pol $\beta$ to remove a $\mathrm{T} / \mathrm{G}$ mismatch in $\mathrm{CpGs}$ and ensure that the polymerase inserts a correct nucleotide. Thus, sufficient amounts of APE1 in mammalian cells should lead to prevention of $\mathrm{C}$ to $\mathrm{T}$ transition mutations resulting from DNA demethylation during BER, thereby sustaining epigenome stability.

Our results showed that pol $\beta$ efficiently extended a mismatched $\mathrm{T}$ basepaired with a template $\mathrm{G}$ that is adjacent to a single-nucleotide gap. This is consistent with the findings from a study from the Wilson group (Sassa et al., 2012). A study from the Sweasy group has shown that pol $\beta$ exhibits low fidelity in gap-filling and strand displacement synthesis (Shah et al., 2003). Pol $\beta$ can extend a $\mathrm{T}$ basepaired with a $\mathrm{G}$ adjacent to a $6 \mathrm{nt}$ gap, albeit with a low efficiency compared with extension of a matched C (Shah et al., 2003). All these findings indicate that pol $\beta$ can tolerate a 3'-terminus mismatch next to a single-nucleotide gap. A translesion synthesis polymerase, pol $\kappa$, has been found to extend a 3'-mismatched $\mathrm{T}$ via a primer-template misalignment or direct extension (Wolfle et al., 2003). However, our results showed that pol $\beta$ could extend a mismatched $\mathrm{T}$ only by direct extension, suggesting that pol $\beta$ interacted with the 3'-mismatched $\mathrm{T}$ and the template strand in a more rigid manner than pol $\kappa$.

Our study has also provided new insight into a mechanism underlying mutations in CGIs that can be readily generated through BER and BER-mediated DNA demethylation. We have demonstrated that in the scenario where 3'-mismatched $\mathrm{T}$ is adjacent to an abasic site in a $\mathrm{CpG}$ dinucleotide, the mismatched $\mathrm{T}$ was efficiently removed by APE1 before pol $\beta$ extended it (Figure 2.7B), and a C to T mutation was prevented (Figure 2.7B). However, in the scenario where a $\mathrm{T}$ occurs at the template strand and is opposite an abasic site 
(Figures 2.2-2.5 and Figure 2.7), the T could not be removed by APE1 3'-5' exonuclease. Instead, pol $\beta$ filled in the gap by inserting an A to basepair with the template $\mathrm{T}$ leading to a $\mathrm{C}$ to $\mathrm{T}$ transition. Thus, in this scenario, $\mathrm{C}$ to $\mathrm{T}$ mutations cannot be prevented by APE1 3'-5' exonuclease. Our discoveries provide new mechanistic evidence underlying the high frequency of mutations at CGIs. In conclusion, our study demonstrates that APE1 exonuclease activity functions as a proofreader for pol $\beta$ to sustain the integrity of CGIs during BER and DNA demethylation by removing a 3'-terminus T/G mismatch. 


\section{CHAPTER 3}

ENVIRONMENTALLY-INDUCED OXIDATIVE DNA DAMAGE DISRUPTS DNA METHYLATION PATTERN IN HUMAN BREAST CANCER 1 (BRCA1) GENE VIA BASE EXCISION REPAIR

\section{ABSTRACT}

Mutations of breast cancer 1 (BRCA1) gene have been shown to play a crucial role in breast cancer development. Yet, downregulation of BRCA1 expression has commonly been observed in breast cancer cells, and this may result from disruption of DNA methylation pattern in the promoter region of the gene. We examined the effects of environmentally induced oxidative DNA damage on the DNA methylation pattern of BRCA1 gene with bisulfite DNA sequencing. We discovered that environmental oxidative DNA damaging agents, bromate and chromate disrupt DNA methylation pattern at CpG dinucleotides in the BRCA1 gene that range from -189 to +27 in human cells. We found that bromate and chromate resulted in the demethylation of the 5-methylcytosine $(5 \mathrm{mC})$ in the CpGs at the sites of $-189,-134,-29,-19,+16,+19$. Surprisingly, we found that the DNA damaging agents created a series of new DNA methylation sites at the CpGs located at the sites of $-80,-55,+8,+27$ in BRCA1 gene. We showed that bromate and chromateinduced demethylation of $5 \mathrm{mC}$ at the CpGs was mediated through deletion of cytosines and base substitutions via BER, and this was accomplished by nucleotide misinsertion of pol $\beta$ on the CpGs. Moreover, we identified an interaction between DNA polymerase $\beta$ with DNA methyltransferase 1 in cells. Our results indicate that bromate and chromate alter DNA methylation pattern in the promoter and transcribed region of BRCA1 gene via 
oxidative DNA damage. We further suggest that BER crosstalks with DNA methyltransferases to induce additional DNA methylation sites.

\section{INTRODUCTION}

Methylation of DNA is associated with important biological functions including silencing of retrovirus, gene expression, genomic imprinting, X-chromosome inactivation, embryonic development, genomic instability, cancer development, among others (Bird, 1992, 2002; Ehrlich, 2005; Kass et al., 1997; Smith and Meissner, 2013). In mammalian cells, 60 90\% of cytosines in $\mathrm{CpG}$ dinucleotide clusters are methylated at its 5-carbon (Ehrlich et al., 1982). Unmethylated CpG dinucleotide clusters usually locate at the promoter (the upstream) regions of the genes in a tissue-specific manner. A normal DNA methylation pattern at a specific gene is maintained upon the balance between DNA methylation and demethylation, and the underlying mechanisms remain to be elucidated (Niehrs, 2009). While DNA methylation can be modulated by the activities of DNA methylation transferases and supplies of the methylation cofactor S-adenomethionine (SAM), in general, DNA demethylation is mediated by both passive and active pathways in mammalian cells (Gong and Zhu, 2011) and can be modulated by the inhibition of the activities of methyltransferases as well as altered activities of BER enzymes and their coordination. Since Cs and Gs in CpGs form hotspots of oxidative DNA base damage and mutations (Lutsenko and Bhagwat, 1999), DNA base lesions can directly lead to deamination of $5 \mathrm{mCs}$ resulting in loss of the methylated Cs through BER leading to substitution of a $\mathrm{C}$ with other nucleotides and mutations. In addition, a base lesion that occurs on CpGs and the adjacent regions may also affect the activity of DNMTs and even 
inhibit their activities. All these can lead to passive loss of a $5 \mathrm{mC}$, thereby disrupting a normal DNA methylation pattern.

The Environmental toxicant Chromium, Cr (VI) has been reported to be associated with lung cancer (Zhou et al., 2009). The Cr (VI) can also alter the DNA methylation pattern and histone modifications, thereby activating or suppressing gene expression. This may lead to carcinogenesis (Salnikow and Zhitkovich, 2008; Sun et al., 2009; Zhou et al., 2009). Bromate, on the other hand, is an environmental pollutant that can be generated during water disinfection and beverage fermentation (Ballmaier and Epe, 2006). Bromate can induce oxidative DNA damage such as 8-oxoGs (Kawanishi and Murata, 2006) at CpGs that may in turn inhibit the activities of DNMTs causing loss of 5mCs. Since BER mediates active DNA demethylation while it repairs DNA base lesions at CpGs simultaneously, BER may result in mutations via nucleotide misinsertions by pol $\beta$ during these processes because of a low fidelity of DNA synthesis of the DNA polymerase (Jiang et al., 2015). This can also lead to loss of 5mCs and passive DNA demethylation. Thus, DNA base lesions can modulate DNA methylation patterns through different mechanisms.

As one of the most prevalent cancers among American women, breast cancer has been shown to be associated with mutations in the tumor suppressor genes, BRCA1 and BRCA2 (Bosviel et al., 2012; Miki et al., 1994; Wooster et al., 1995). However, recent studies have demonstrated that more than $90 \%$ of breast cancer cases do not have a BRCA1/2 mutation (sporadic breast cancer). However, downregulation of BRCA1 gene expression has been found to be associated with sporadic tumor development (Bosviel et al., 2012; Dobrovic and Simpfendorfer, 1997; Esteller et al., 2000). It appears that the DNA hypermethylation at the promoter of the BRCA1 gene may play a critical role for 
suppressing of the gene expression in sporadic breast cancer (Esteller et al., 2000; Herman and Baylin, 2003; Jones and Baylin, 2007; Matros et al., 2005; Rice et al., 1998; Rice et al., 2000; Wei et al., 2005). On the basis of these studies, we hypothesized that the environmental toxicants, bromate and chromate, can cause DNA base lesions in the BRCA1 promoter as well as the encoding region of BRCA1 protein, and this then initiates BER that may subsequently modulate DNA methylation pattern at the BRCA1 gene. This may then result in downregulation of BRCA1 gene expression, leading to development of breast cancer. To test this hypothesis, in this study, we examined the effects of bromate and chromate on the DNA methylation pattern of BRCA1 gene in human embryonic kidney 293 (HEK293) cells treated with different concentrations of the environmental toxicants using bisulfite DNA sequencing. We found that bromate and chromate not only altered the DNA methylation pattern at the BRCA1 gene, but also caused mutations in the gene. We found that bromate and chromate resulted in the loss of $5 \mathrm{mCs}$ as well as $\mathrm{C}$ to $\mathrm{G}$ and $\mathrm{C}$ to $\mathrm{A}$ mutations at several $\mathrm{CpGs}$ of the BRCA1 gene. We further demonstrated that the mutations were caused by pol $\beta$ nucleotide misinsertion during its synthesis in bypassing a base lesion, 8-oxoG located at the template strand. This was further supported by the fact that pol $\beta$ was recruited to DNA damaging sites induced by bromate and chromate in HEK293 cells. Surprisingly, we found that bromate and chromate also induced additional DNA methylation sites at the BRCA1 gene, and pol $\beta$ interacted with DNMT1. This suggests that pol $\beta$ recruited DNMT1 to the sites that surround a DNA base lesion. This may stimulate DNA methylation by DNMT1 at the sites adjacent to the damaging sites. Our study provides new insights into the molecular mechanisms underlying oxidative DNA 
damage induced alterations of DNA methylation at BRCA1 gene and its effects on epigenetic changes of the BRCA1 gene and its expression.

\section{MATERIALS AND METHODS}

\subsection{Materials}

Human embryonic kidney (HEK) cells were purchased from Life Technologies (Carlsbad, CA, USA). The fetus bovine serum (FBS), RPMI 1640 and Dulbecco's Modified Eagle's Medium (DMEM) cell culture medium were purchased from Thermo Fisher Scientific (Waltham, MA, USA). The genomic DNA isolation kit was purchased from Promega (Agora, WI, USA). The Lightning Bisulfite Conversion kit was purchased from ZYMO Research (Irvine, CA, USA). The Dream Taq polymerase master mix and the Original TA Cloning kit were purchased from Invitrogen (Carlsbad, CA, USA). The Rapid DNA Ligation kit was purchased from Thermo Scientific (Carlsbad, CA, USA), and the BigDye kit for sequencing was purchased from Applied Biosystems (Austin, TX, USA).

The DNA oligonucleotides containing an 8-oxoguanine were synthesized by the Midland Certified Reagent Company Inc. (Midland, TX, USA). All other oligonucleotides were synthesized by Integrated DNA Technologies (IDT, Coralville, IA, USA). Deoxynucleoside 5'-triphosphates (dNTPs) were purchased from Sigma-Aldrich (St. Louis, MO, USA). Radionuleotides, $\left[\gamma_{-}{ }^{32} \mathrm{P}\right]$ ATP $(6000 \mathrm{mCi} / \mathrm{mmol})$ and cordycepin 5 'triphosphate $3^{\prime}-\left[\alpha^{-}{ }^{32} \mathrm{P}\right](5000 \mathrm{mCi} / \mathrm{mmol})$ were purchased from MP Biomedicals (Santa Ana, CA, USA). Micro Bio-Spin 6 chromatography columns were from Bio-Rad (Hercules, CA, USA). All other standard chemical reagents were purchased from Thermo Fisher Scientific (Pittsburgh, PA, USA) and Sigma-Aldrich (St. Louis, MO, USA). Pol $\beta$ were purified by FPLC as described previously (Liu et al., 2005). The pol $\beta$ expression 
plasmid was a generous gift from Dr. Samuel H. Wilson at the National Institute of Environmental Health Sciences (NIEHS)/National Institutes of Health, Research Triangle Park, North Carolina.

Polyclonal anti-pol $\beta$ primary antibody (ab26343), monoclonal anti-pol $\beta$ primary antibody (ab175197), polyclonal anti-DNMT1 primary antibody (ab19905), and monoclonal anti-DNMT1 primary antibody (ab188453) were purchased from Abcam (Cambridge, MA, USA). Pierce protease inhibitor tablets (B2162578) and protein A agarose beads were purchased from Thermo Scientific (Waltham, MA, USA). The LI-COR C-DiGit Chemiluminescence Western Blot Scanner (Model: 3600) for western blot was purchased from LI-COR Biotechnology (Lincoln, NE, USA)

\subsection{Oligonucleotide substrates}

Substrates containing a 76-nt template strand with an 8 -oxoG or THF at the $39^{\text {th }}$ Table 3.1 Sequences of the oligonucleotide substrates

\begin{tabular}{lrl}
\hline $\begin{array}{l}\text { Downstream } \\
\text { strand }\end{array}$ & nt & Seq. 5'-3' \\
\hline D1 & 37 & pGAGCTCACGCCGCGCAGTCGCAGTTTTAATTTATCTG \\
D2 & 37 & pFGAGCTCACGCCGCGCAGTCGCAGTTTTAATTATCTG \\
\hline $\begin{array}{l}\text { Template } \\
\text { strand }\end{array}$ & & \\
\hline T1 & 76 & CAGATAAATTAAAACTGCGACTGCGCGGCGTGAGCTCG \\
T2 & $76 \begin{array}{l}\text { CTGAGACTTCCTGGACGGGGGACAGGCTGTGGGGTTC } \\
\text { CAGATAATTAAAACTGCGACTGCGCGGCGTGAGCTC (8- } \\
\text { oxoG) }\end{array}$ \\
\hline $\begin{array}{ll}\text { Upstream } \\
\text { strand }\end{array}$ & & \\
\hline U1 & 38 & GAAACCCCACATCCTGGACGGGGGACAGGCTGTGGGGTTTC \\
\hline
\end{tabular}

nucleotide counted from the 5 '-end were designed to mimic a DNA base lesion generated after the genome is damaged oxidative DNA damaging agent. Substrates mimicking the 
intermediates with a 1-nt gap opposite a template 8-oxoG formed during DNA lagging strand synthesis and BER, were constructed by annealing the 38-nt upstream primer and a 37-nt downstream primer with a 5'-phosphate or 5'-THF residue with the template strand containing a G or 8-oxoG at a molar ratio of 1:2:2. Substrates were radiolabeled at the 5'end of the upstream primers for measuring the activities of different enzymes. The sequences of the oligonucleotide substrates are listed in Table 3.1.

\subsection{Treatment of HEK293 cells by bromate and chromate}

The HEK293 cells were grown in DMEM with 10\% FBS to near confluence. Cells were then exposed to $5 \mathrm{mM}$ bromate or $10 \mu \mathrm{M}$ of chromate for 24 hours. Cells were washed by PBS twice and supplied with the whole medium (10\% FBS) for additional 24 to 48 hours for recovery and DNA damage repair. Cells were then harvested, and the genomic DNA was isolated according to the protocol provided by the Promega genomic DNA isolation kit. Genomic DNA was then subject to bisulfite conversion with Bisulfite Lightning Conversion kit purchased from ZYMO Research. The DNA fragments in BRCA1 gene were amplified. The bisulfite PCR reactions were set up by using the primers shown in Table 3.2.

Table 3.2 Primers for bisulfite sequencing

\begin{tabular}{lcc}
\hline $\begin{array}{l}\text { Sense } \\
\text { strand }\end{array}$ & nt & Seq. 5'-3' \\
\hline Forward & 36 & GAGGCTAGAGGGCAGGCACTTTATGGCAAACTCAGG \\
Reverse & 25 & GTCCCCCGTCCAGGAAGTCTCAGCG \\
\hline Anti-sense & \\
strand & \\
\hline Forward & 38 & TTTTAGAATAYGAAATTAAGGTATAATTAGAGGATGGG \\
Reverse & 37 & CAATAAACCRCAACTAAAAAAATAAAAACTAAAAAAC \\
\hline Y: either C/T; R: either G/A
\end{tabular}




\subsection{Recruitment of pol $\beta$ to DNA damage sites}

The HEK293 cells were grown in DMEM with 10\% FBS to 70 80\% confluence. Cells were then transfected with a plasmid that expresses a green fluorescence protein tagged human pol $\beta$ (GFP-hPol $\beta$ ) 24 hours. Cells were then seeded in a chamber slide and grown for 24 hours. Cells were subsequently treated with $5 \mathrm{mM}$ bromate or $10 \mu \mathrm{M}$ chromate for $1 \mathrm{~h}$ and fixed with 4\% paraformaldehyde. Cells were washed twice by ice cold PBS and stained by mounting media containing $1 \%$ of DAPI and were imaged with the Olympus Laser Confocal Microscope (FIU Confocal Microscopy Facilities).

\subsection{Pol $\beta$ DNA synthesis in bypassing an 8-oxoG}

In vitro pol $\beta$ DNA synthesis in bypassing an 8-oxoG on the template strand that is opposite the $\mathrm{C}$ at $-166 \mathrm{nt}$ of the BRCA1 promoter was performed by incubating purified human pol $\beta$ with the substrates bearing aG or an 8 -oxoG opposite the $\mathrm{C}$ at $-166 \mathrm{nt}$ strand that is complementary to the strand ranging from -189 to $-80 \mathrm{nt}$ of the BRCA1 promoter. Various concentrations of pol $\beta$ and substrates were incubated in BER reaction buffer with $5 \mathrm{mM} \mathrm{MgCl}_{2}$, and $50 \mu \mathrm{M}$ dNTPs in $10-\mu \mathrm{L}$ reaction mixture. Reaction mixtures were incubated at $37^{\circ} \mathrm{C}$ for $30 \mathrm{~min}$. Reactions were terminated with $2 \mathrm{x}$ stopping buffer that contains $95 \%$ formamide and $10 \mathrm{mM}$ EDTA followed by incubation at $95^{\circ} \mathrm{C}$ for $5 \mathrm{~min}$. All substrates were ${ }^{32} \mathrm{P}$-labeled at the 5 '-end of the upstream strand. Substrates and products were separated with $15 \%$ or $18 \%$ urea-denaturing PAGE gel and were detected with the Pharos FX Plus PhosphorImager (Bio-Rad, Hercules, CA, USA)

\subsection{Pol $\beta$ nucleotide misinsertion}

In vitro pol $\beta$ misinsertion activity was performed by incubating various concentrations of pol $\beta$ with the substrates containing a normal $\mathrm{G}$ or 8 -oxoG on the 
template. The $10-\mu \mathrm{L}$ reaction was assembled with the substrates and different concentrations of pol $\beta$ in BER reaction buffer with $5 \mathrm{mM} \mathrm{MgCl}_{2}$ and $50 \mu \mathrm{MdA}$ or dT or $\mathrm{dG}$ or $\mathrm{dC}$, respectively. Reaction mixtures were assembled on ice and incubated at $37^{\circ} \mathrm{C}$ for $30 \mathrm{~min}$. Reactions were terminated by addition of $2 \times$ stopping buffer containing $95 \%$ formamide and $10 \mathrm{mM}$ EDTA and incubation at $95 \%$ for $5 \mathrm{~min}$. Substrates and products were then separated by $15 \%$ or $18 \%$ urea-denaturing PAGE gel and detected with Pharos

FX Plus PhosphorImager. All substrates were ${ }^{32} \mathrm{P}$ labeled at the 5 '-end of the upstream primers.

\subsection{Pol $\beta$-DNMT1 interaction detected by co-immunoprecipitation (Co-IP) to detect in HEK293 cells after bromate/chromate exposure}

The interaction been DNMT1 and pol $\beta$ was examined by employing Co-IP assay. The HEK293 cells were treated with $10 \mu \mathrm{M}$ chromate for 24 hours with or without 24 hours of recovery. Cells were harvested in cold PBS buffer, then resuspended in cold lysis buffer with $0.1 \%$ NP40 with protease inhibitors (Thermo Scientific, Waltham, MA, USA). $20 \mu \mathrm{L}$ of cell lysate was taken and set aside as an input. $800 \mathrm{ng}$ of cell lysate was precleared with $20 \mu \mathrm{L}$ of protein A agarose beads (Thermo Scientific, Waltham, MA, USA) (50\% protein A agarose at a ratio of $100 \mu \mathrm{L}$ for a $1 \mathrm{~mL}$ lysate) for $30 \mathrm{~min}$ at $4^{\circ} \mathrm{C}$. The amount of total protein of cell lysate was determined with Bradford assay. Cell lysate was then diluted with PBS to the total protein of $5 \mu \mathrm{g} / \mu \mathrm{L}$. Seventy five microliters cell lysate was incubated with $3 \mu \mathrm{L}$ of polyclonal anti-pol $\beta$ primary antibody (Abcam, Cat. No. ab26343, Cambridge, MA) at $4{ }^{\circ} \mathrm{C}$ overnight. Cell lysate was then incubated with $100 \mu \mathrm{L} 50 \%$ protein A agarose beads at $4^{\circ} \mathrm{C}$ with rotation for 2 hours and subject to centrifugation at $14,000 \mathrm{~g}$ for $1 \mathrm{~min}$ 
at $4^{\circ} \mathrm{C}$ for agarose bead precipitation. The beads were washed 3 times with wash buffer containing $20 \mathrm{mM}$ HEPES, $\mathrm{pH}$ 7.5, $150 \mathrm{mM} \mathrm{NaCl}, 1 \% \mathrm{NP}-40$ and $2 \mathrm{mM}$ EDTA. Protein complexes precipitated with the antibody were eluted in SDS-loading buffer with heating at $50{ }^{\circ} \mathrm{C}$ for 10 mins. Individual proteins in the precipitated protein complexes were then subjected to SDS-PAGE and immunoblotting with monoclonal anti-pol $\beta$ antibody (1:2000, ab175197, Abcam) and monoclonal anti-DNMT1 primary antibody (1:2000, ab188453, Abcam). Western Sure Premium secondary antibody (1:2000, 926-80011, LICOR) was employed for detecting the proteins with measurement of chemiluminescence that was detected by LI-COR C-DiGit scanner.

\section{RESULTS}

\subsection{Bromate and chromate treatment altered DNA methylation pattern on BRCA1} gene

Since bromate and chromate-induced oxidative DNA damage can change the DNA methylation pattern and histone post-translational modifications by targeting Cs, 5mCs and Gs on CpGs, it is conceivable that these toxicants can also cause oxidized bases on CpGs at BRCA1 gene and subsequently the loss of $5 \mathrm{mCs}$ and point mutations, thereby affecting activation and/or repression of BRCA1 gene expression as well as breast cancer development. To test this, we initially determined the DNA methylation pattern on the promoter and encoding region of BRCA1 gene in untreated HEK293 cells and HEK293 cells treated with bromate and chromate using bisulfite DNA sequencing (Figures 3.1A and 3.2A). The results showed that in the promoter region of BRCA1 gene of untreated cells, the CpGs located at $-189,-134,-29,-19,+16$, and +19 on the sense 
strand of the gene are partially methylated (Figures 3.1A and 3.2A). On the antisense strand, the CpGs located at -189 , and -80 are partially methylated (Figure $3.2 \mathrm{~A}$ ).

However, cellular treatment of $5 \mathrm{mM}$ bromate for $24 \mathrm{~h}$ led to the formation of additional methylation sites at the CpGs located at $-80,-55,-21$, and +8 (Figures 3.1B and 3.2B). The treatment also resulted in the loss of methylation at $-189,-134,+16$ and +19 (Figures 3.1B and 3.2B). Chromate treatment at $10 \mu \mathrm{M}$ led to the formation of new methylation sites at $-80,-55$, and +27 while it resulted in loss of DNA methylation at $-189,-29,-19$, +16 , and +19 (Figures $3.1 \mathrm{C}$ and $3.2 \mathrm{C}$ ). 

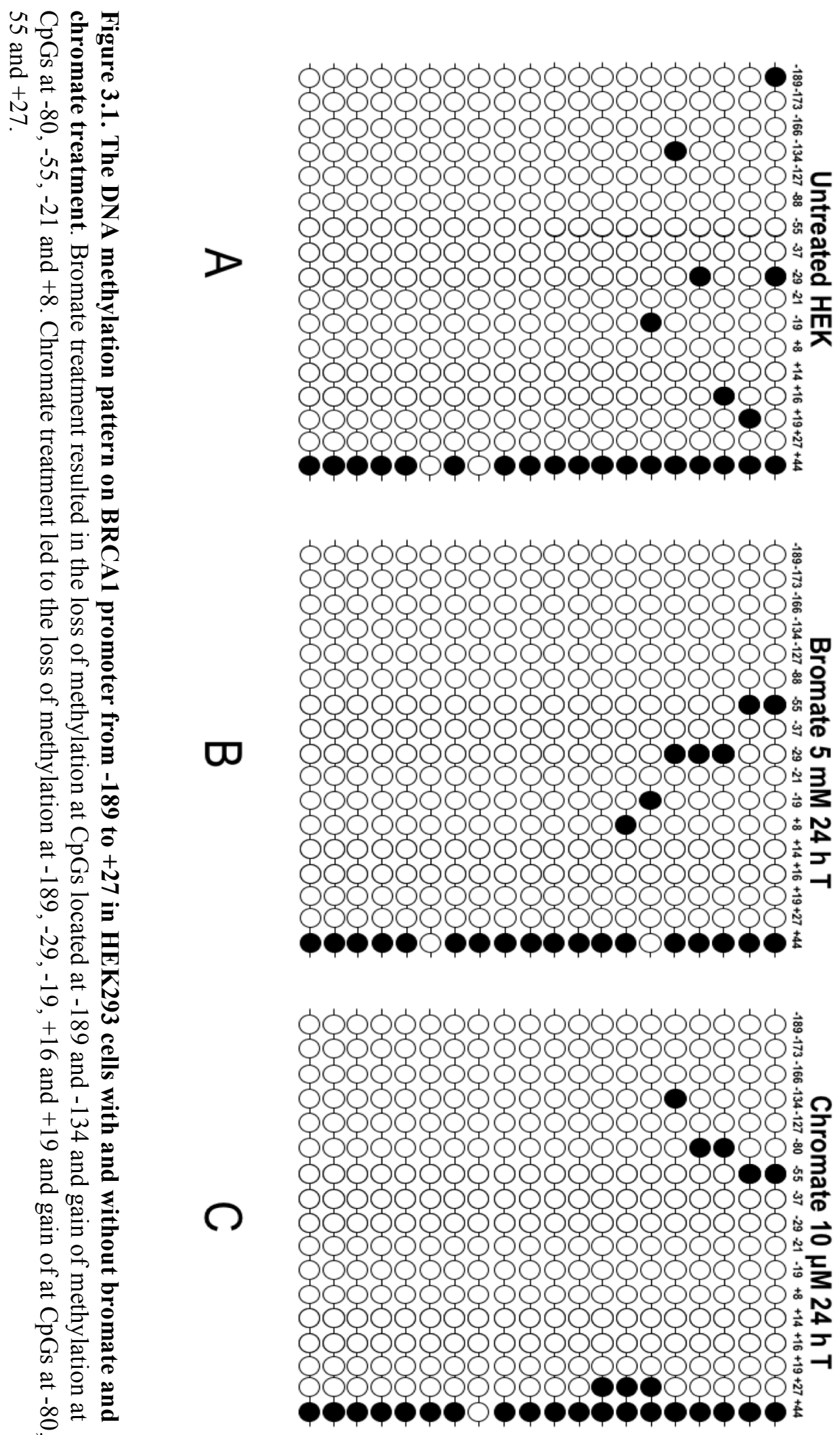


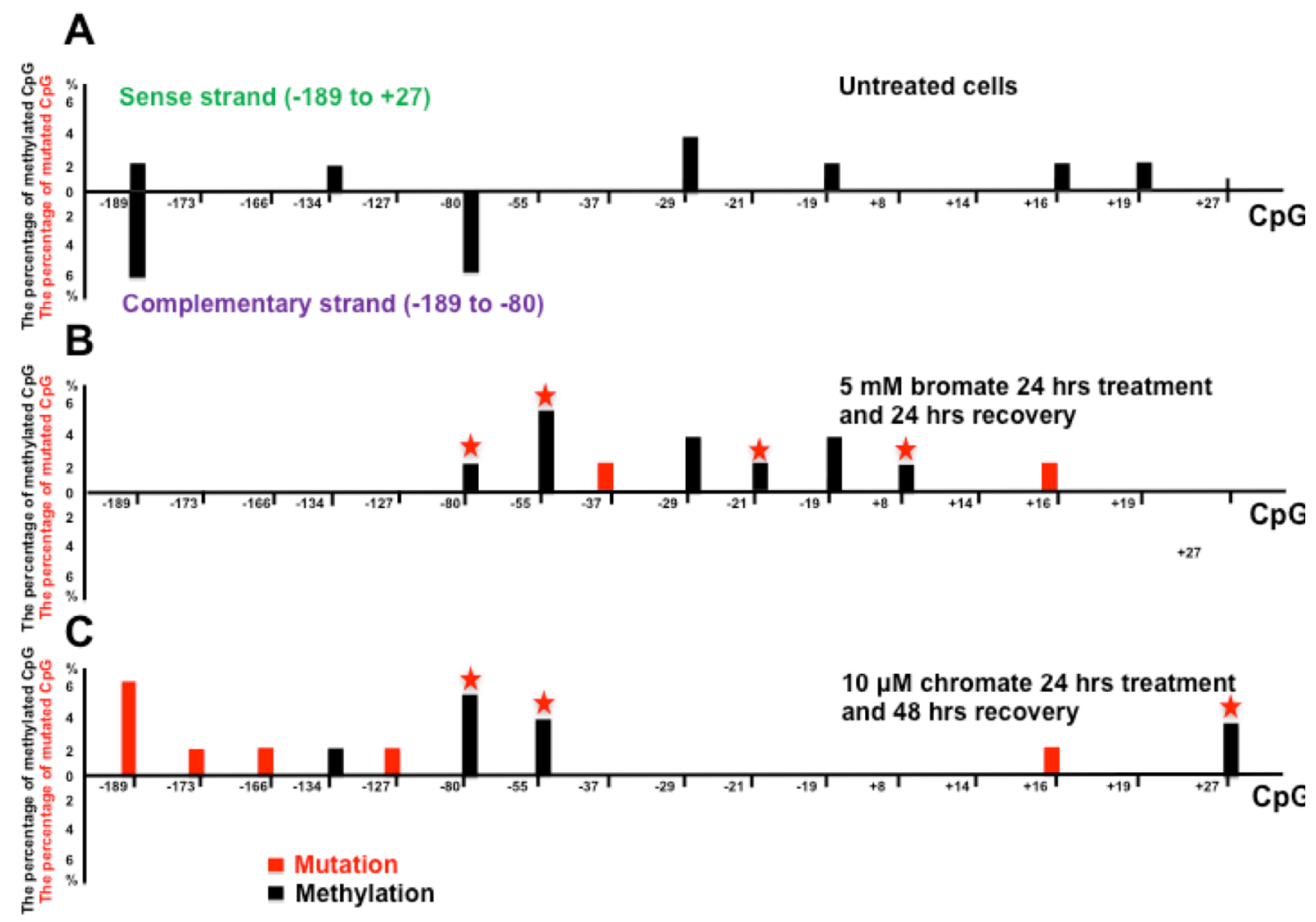

Figure 3.2 The DNA methylation pattern on BRCA1 gene promoter and encoding region from -189 to +27 in HEK293 cells with or without treatment of bromate and chromate.

Bromate and chromate treatment caused the loss of $5 \mathrm{mCs}$ at the CpGs located at -34 and +16 via mutations. Further mutation analysis showed that bromate treatment resulted in $\mathrm{C}$ to A mutation (Figure 3.3B), whereas chromate treatment mainly led to $\mathrm{C}$ to $\mathrm{G}$ mutation at $-189,-173,-166$, and -127 , (Figure 3.3A). The mutation spectrum showed that the major mutations induced by bromate and chromate on the BRCA1 gene is $\mathrm{C}$ to $\mathrm{G}$ mutation (Figure 3.3). 


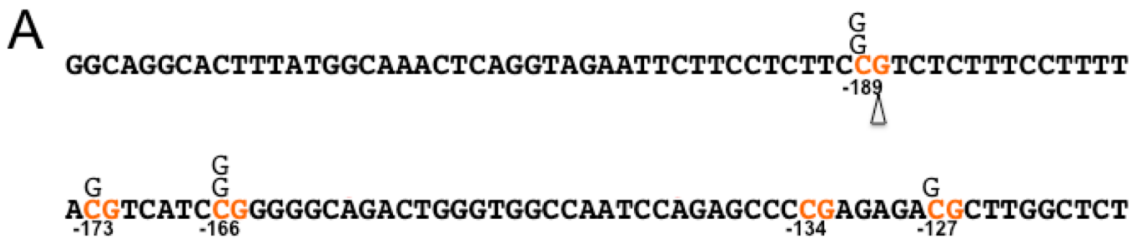

TTCTGTCCTCCCATCCTCTGATTGTACCTTGATTCGTATTCTGAGAGGCTGCTGCT

$\underset{-55}{\operatorname{TAGCGTAGCCCCTTGGTTTCCGTGGCAACGGAAAAGCGCGGAATTACAGATAAATT~}}$

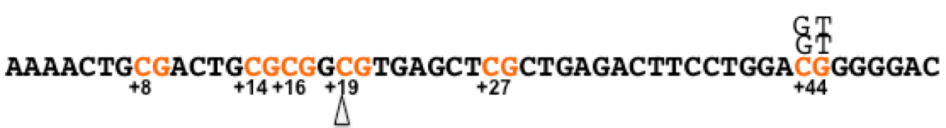

B GGCAGGCACTTTATGGCAAACTCAGGTAGAATTCTTCCTCTTCCGTCTCTTTCCTTTT $-189$

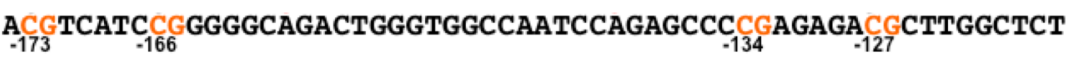
TTCTGTCCCTCCCATCCTCTGATTGTACCTTGATTTCGTATTCTGAGAGGCTGCTGCT $\underset{-55}{\text { TAGCGAGCCCCTTGGTTTCCGTGGCAACGGAAAAGCGCGGAATTACAGATAAATT }} \underset{-37}{\text { A }}$

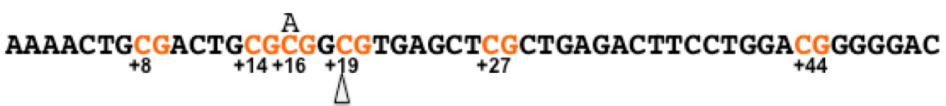

Figure 3.3 The mutation spectrum of BRCA1 gene in HEK293 cells induced by chromate (A) and bromate (B) treatment. 


\subsection{Pol $\beta$ recruits to the DNA damage sites induced by bromate and chromate}

To determine whether pol $\beta$ can be recruited to bromate and chromate-induced DNA damage sites in cells, we exposed HEK293 cells transfected with a plasmid expressing GFP-tagged human pol $\beta$ to $5 \mathrm{mM}$ bromate. We found that GFP-pol $\beta$ proteins were located in the nucleus stained with DAPI (Figure 3.6 upper panel). Upon treatment of chromate, the green loci of GFP-pol $\beta$ formed in the nucleus of HEK293 cells. This indicates that

\section{Untreated sample}
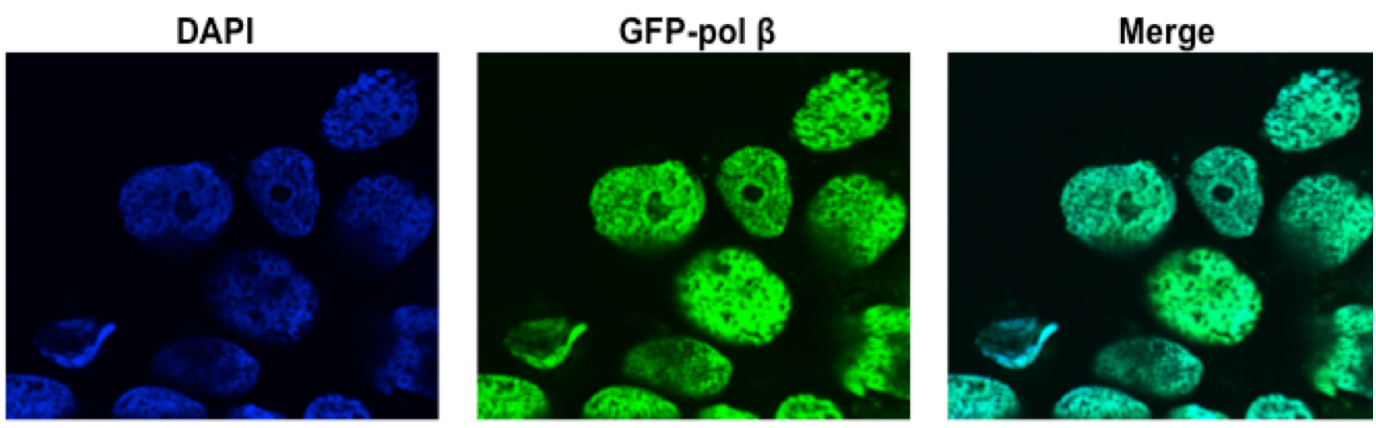

$10 \mu \mathrm{M}$ chromate 1 hour exposure
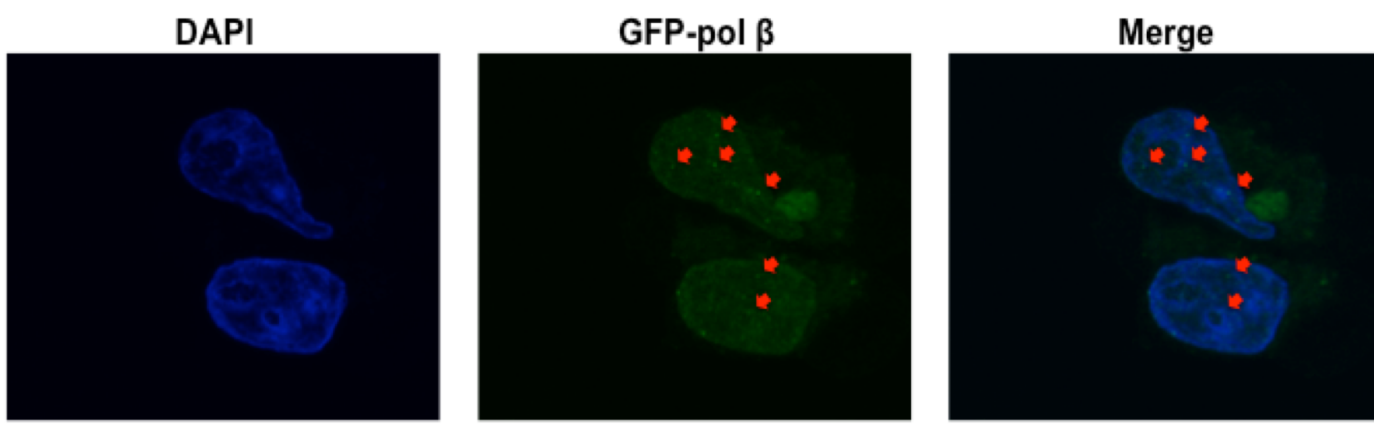

Figure 3.4 HEK293 cells transfected with GFP-pol $\beta$ were treated with $10 \mu M$ chromate for 1 hour. The green foci of pol $\beta$ inside the nucleus pointed by the yellow arrows. The HEK293 cells were transfected with a plasmid expressing GFP-tagged pol $\beta$ for 24 hours.

GFP-pol $\beta$ was recruited to DNA damage induced by chromate (Figure 3.4, bottom panel). 


\subsection{Pol $\beta$ can bypass an 8-oxoG during DNA replication and BER through nucleotide}

misinsertion.

Previous studies have shown that during DNA replication and BER, pol $\beta$ exhibits translesion synthesis activity to bypass a DNA base lesion (Bassett et al., 2002b; Chary et al., 2012b; Hashim et al., 1997a; Hoffmann et al., 1996a; Maga et al., 2009a; Servant et al., 2002a; Vaisman and Chaney, 2000a; Villani et al., 2011a). Pol $\beta$ lesion bypass synthesis can further induce a high frequency of mutations in the genome (Bassett et al., 2002a; Batra et al., 2006; Beard et al., 2009; Beard and Wilson, 2014; Chary et al., 2012a; Efrati et al., 1997; Hashim et al., 1997b; Hoffmann et al., 1996b; Servant et al., 2002b; Vaisman and Chaney, 2000b). Furthermore, since pol $\beta$ does not bear a 3'-5' exonuclease activity for proofreading, high levels of pol $\beta$ may cause high mutation rates, and this is associated with development of a variety of cancer cells (Canitrot et al., 2000; Scanlon et al., 1989; Srivastava et al., 1999). Thus, it is possible that pol $\beta$ was recruited to DNA damage induced by bromate and chromate to bypass an 8 -oxoG through its translesion synthesis activity. To test this, we examined whether pol $\beta$ can bypass an 8 -oxoG through nucleotide misinsertation to create a G:G mispair at the promoter region of BRCA1. This was determined by incubating $20 \mathrm{nM}$ purified pol $\beta$ protein with the substrate without the downstream strand (open template) and the gapped substrate that contained a template 8oxoG at $\mathrm{CpG}$ located at -166 of human BRCA1 promoter. The results showed that $20 \mathrm{nM}$ pol $\beta$ inserted all four dNTP to base pair with the 8 -oxoG on the open template substrate (Figure 3.5A and 3.5, lanes 2, 3, 4 and 5) but with a higher efficiency in inserting $\mathrm{dA}$ and $\mathrm{dC}$ (Figure 3.5, lanes 2 and 4). For the 1-nt gap-THF substrate containing an 8-oxoG, 20 

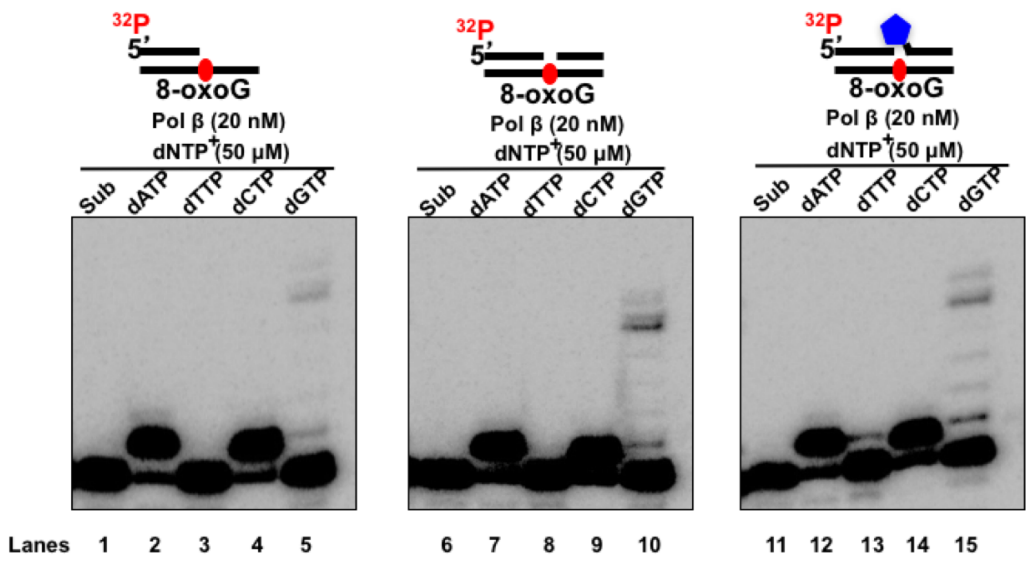

Figure 3.5 Pol $\boldsymbol{\beta}$ misincorporated nucleotides to bypass an 8-oxoG. Lanes 2,7 and 12 correspond to reaction mixtures with $20 \mathrm{nM}$ pol $\beta$ (panel B) in the presence of $50 \mu \mathrm{M}$ dA. Lanes 3,8 and 13 correspond to reaction mixtures with $5 \mathrm{nM}$ pol $\beta$ (panel A) and $20 \mathrm{nM}$ pol $\beta$ (panel $\mathrm{B}$ ) in the presence of $50 \mu \mathrm{M} \mathrm{dT}$. Lanes 4,9 , and 14 correspond to reaction mixtures with $20 \mathrm{nM}$ pol $\beta$ (panel B) in the presence of $50 \mu \mathrm{M} \mathrm{dC}$. Lanes 5,10 and 15 correspond to reaction mixtures with $20 \mathrm{nM}$ pol $\beta$ (panel B) in the presence of $50 \mu \mathrm{M} \mathrm{dG}$. Substrates are illustrated schematically above the gel.

$\mathrm{nM}$ pol $\beta$ mainly inserted $\mathrm{dA}$ and $\mathrm{dC}$ when bypassing the lesion (Figure 3.5, lanes 12, 14). It also inserted $\mathrm{dT}$ and $\mathrm{dG}$ with a relatively low efficiency in bypassing an 8-oxoG (Figure 3.5 , lanes 13,15$)$. Interestingly, the results showed pol $\beta$ preferentially to insert $\mathrm{dG}$ when bypassing an 8-oxoG in the open template, 1-nt gap and 1-nt gap-THF substrates (Figure 3.5, lanes 5, 10, and 15). Furthermore, pol $\beta$ exhibited a higher efficiency to insert dG when bypassing an 8-oxoG in the 1-nt gap-THF substrate compared to the 1-nt gap substrate. The results indicate that pol $\beta$ misinserted dG to bypass an 8-oxoG lesion during BER in CpGs. This is consistent with our results showing that bromate and chromate predominantly induced a $\mathrm{C}$ to $\mathrm{G}$ mutation via a $\mathrm{G}: \mathrm{G}$ mispair (Figures 3.1 and 3.2).

\subsection{Pol $\beta$ interacts with DNMT1 in HEK293 cells upon chromate treatment}

Previous studies have shown that DNA repair proteins such as HR enzymes can interact with DNMT3a, The BER enzyme TDG can also interact with DNMT3a alteration 
of DNA methylation pattern and gene transcription (Li et al., 2007; Russo et al., 2016). Since our results showed that bromate and chromate induced additional DNA methylation site at BRCA1 gene, this suggests a crosstalk between DNA damage, BER and DNMTs. We then examined a possibility that pol $\beta$ and DNMT1 may interact with each other in cells with co-immunoprecipitation. We found that pol $\beta$ and DNMT1 coimmunoprecipitated upon treatment of $10 \mu \mathrm{M}$ chromate without recovery (Figure 3.6, lane 6) suggesting that pol $\beta$ recruited DNMT to the region adjacent chromate-induced DNA base lesions in cells. Interestingly, pol $\beta$ exhibited little interaction with DNMT1 after 24 $\mathrm{h}$ recovery from chromate treatment (Figure 3.6, lane 7) suggesting that the two proteins interacted immediately after DNA damage occurred at BRCA1 gene.

\section{DISCUSSION}

For the first time, we showed that environmental toxicants, bromate and chromate altered the DNA methylation pattern of the BRCA1 gene by directly causing the loss of $5 \mathrm{mCs}$, mutations and indirectly creating additional new $5 \mathrm{mCs}$. We found that bromate- and chromate-induced oxidative DNA damage resulted in the loss of $5 \mathrm{mCs}$ specifically on CpGs located at $-134,-29,-19$ and +19 as well as point mutations on CpGs at -189 and +16 (Figure 3.2). We further demonstrated that bromate and chromate predominantly caused C to $\mathrm{G}$ and $\mathrm{C}$ to $\mathrm{A}$ mutations (Figure 3.3), and this was mediated by pol $\beta$ nucleotide misinsertion in bypassing an 8-oxoG on the template strand (Figure 3.5). Surprisingly, we discovered that bromate and chromate induced additional methylation on CpGs at $-80,-55$, -21 and +8 , which are adjacent to the sites with loss of $5 \mathrm{mCs}$ and point mutations (Figures 
3.1 and 3.2). We further identified an interaction between pol $\beta$ and DNMT1 (Figure 3.6).

On the basis of the results, we propose a hypothetical model during which bromate and chromate induce oxidative DNA base damage on the CpGs at the BRCA1 gene. Pol $\beta$ is then recruited to the lesions to perform its gap-filling synthesis. This subsequently leads to loss of $5 \mathrm{mCs}$ via substitution of a $5 \mathrm{mC}$ with a $\mathrm{C}$ and mutations at the $\mathrm{CpGs}$ via pol $\beta$ mediated nucleotide misinsertion for bypass of an 8-oxoG on the template strand. Simultaneously, bromate- and chromate-induced base lesions induce the interaction

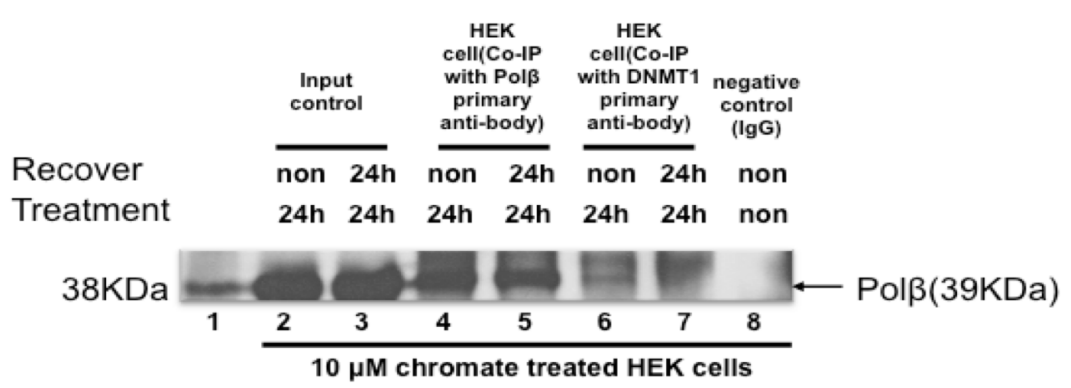

Figure 3.6 Chromate exposure resulted in the interaction between pol $\beta$ and DNMT1. Lane 1 represents molecular weight marker. Lanes 2-3 represent the input control. Lanes 45 illustrate the immunoprecipitates resulting from a pol $\beta$ antibody. Lanes 6-7 represent the immunoprecipitates resulting from a DNMT1 antibody. Lane 8 correspond to the IgG control. Lanes 3, 5, 7 illustrate the samples obtained after $24 \mathrm{~h}$ recovery. Cells were treated with $10 \mu \mathrm{M}$ chromate for $24 \mathrm{~h}$.

between pol $\beta$ and DNMT1, which recruit DNMT1 to create additional methylated CpGs at the BRCA gene (Figure 3.7). 


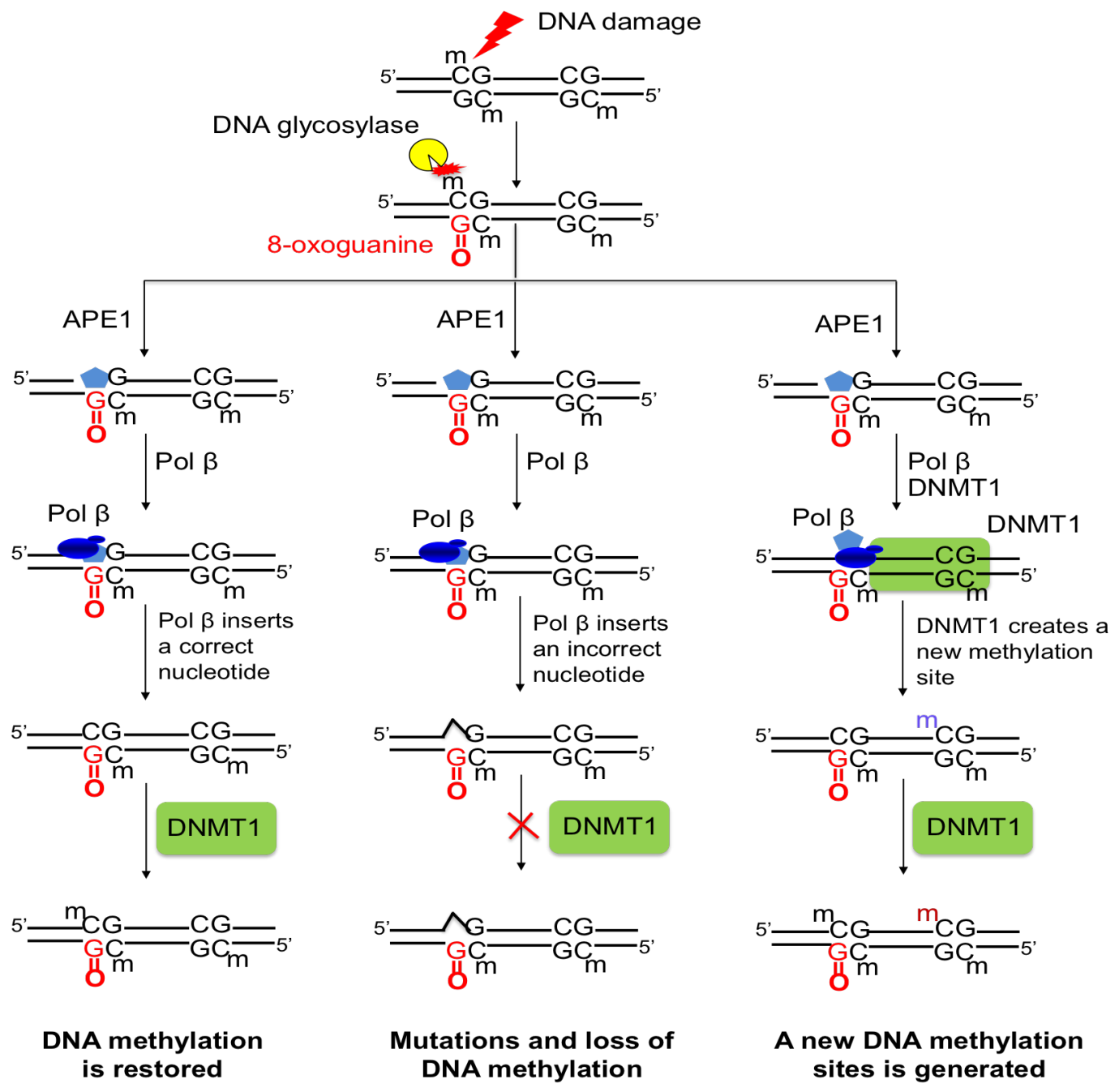

Figure 3.7 The hypothetical model for bromate and chromate to alter DNA methylation pattern of the BRCA1 gene via DNA base lesions and BER in human cells.

Previous studies showed that DNMT1, DNMT3a can interact with DNA repair enzymes such as TDG, therefore alter DNA methylation pattern via DNA damage (Li et al., 2007). Giusi Russo's group has further demonstrated that DSBs also modulate DNA methylation (Russo et al., 2016). However, the mechanisms and biological significance of DNA damage-induced DNA methylation pattern change remain unknown. Since TDG plays a critical role in mediating one of the subpathways of BER-mediated active DNA 
demethylation, the interaction between TDG and DNMTs suggests that the BER-mediated active DNA demethylation coordinates with DNMTs to sustain the stability of DNA methylation. Here, we provided the first evidence showing that a repair DNA polymerase, pol $\beta$ interacted DNMT1 to recruit it to the sites adjacent to a base lesion producing additional 5mCs on the CpGs at BRCA1 gene. The results further suggest that DNA base lesions can modulate DNA methylation pattern of BRCA1 gene by facilitating the interactions between BER enzymes and DNMT1.

Recent studies have shown that oxidative DNA damage can induce the recruitment of DNMT1 and DNMT3B and histone posttranslational modification enzymes to DNA damaging sites, and this has been implicated in inflammation-induced tumorigenesis (Maiuri et al., 2017; O'Hagan et al., 2011). It has been further shown that the mismatch repair proteins, MSH2 and MSH2-MSH6 can be recruited to oxidative DNA damage to recruit DNMT1 to the damaging sites causing DNA methylation pattern changes (Ding et al., 2016; Maiuri et al., 2017). This indicates that mismatch repair proteins may mediate the recruitment of DNMTs to DNA base lesions. Yet, it remains unknown if DNA repair enzymes and proteins from the other DNA repair pathways can interact DNMTs and recruit them to DNA damage sites. Here, we made the first discovery that the central component of BER, pol $\beta$ interacted with DNMT1 to modulate DNA methylation pattern in the BRCA1 gene. Interestingly, we have recently found that MSH2-MSH3 can physically interact with pol $\beta$ in cells (Lai et al., 2016). Thus, it is possible that pol $\beta$ may interact with DNMT1 through MSH2 protein in cells. 


\section{SUMMARY}

DNA base excision repair (BER) is responsible for repairing DNA base lesions such as alkylated, oxidized and deaminated bases as well as ssDNA breaks. Thus, it is an essential repair pathway of maintaining genomic stability and integrity. Moreover, BER plays a critical role in mediating active DNA demethylation thereby exhibiting a dual role in DNA damage repair and regulation of DNA methylation. In addition, BER is also involved in lesion bypass during DNA replication and repair particularly the bypass of cyclodeoxynucleotides $(\mathrm{cdPu})$ lesions that are resistant to DNA repair and readily accumulate in the genome. In Chapter 1 of this dissertation, we explored the mechanisms underlying bypass of cdA lesions by pol $\beta$ during DNA replication and repair. We showed that pol $\beta$ knockout MEF cell extract failed to produce repair product via bypass of a $R$ cdA or $S$-cdA lesion during BER indicating that pol $\beta$ played a major role in bypassing cdA lesions. We demonstrated that pol $\beta$ bypassed a $R$-cdA by incorporating both correct and incorrect nucleotides and failed to bypass a $S$-cdA lesion, thereby resulting in accumulation of DNA strand breaks. This study identified a new role of pol $\beta$, the central component of BER in bypassing cdPu lesions during DNA replication and BER. In Chapter 2, we explored the dual roles of key BER enzymes, pol $\beta$ and APE1 in processing an intermediate formed during active DNA demethylation and a BER intermediate simultaneously in the context of CpGs and their effects on genome integrity. We found that when the BER intermediate, abasic site (AP site) is generated next to the 5-methylcytosine deamination product, a T/G mismatch, TDG failed to remove the mismatched T. Yet, the abasic site could still be efficiently incised by APE1 leading to a 3'-mismatched T. We 
found that pol $\beta$ efficiently extended the 3'-mismatched T resulting in the sustainment of a $\mathrm{C}$ to T mutation. However, we found that high concentrations of APE1 3'-5' exonuclease activity efficiently removed the mismatched $\mathrm{T}$ before pol $\beta$ could extend the 3 '-mismatched $\mathrm{T}$, thereby preventing the $\mathrm{C}$ to $\mathrm{T}$ mutation. We found that APE1 3'-5' exonuclease cleaved the mismatched T 10-fold faster than pol $\beta$ extended the 3'-mismatched T during BER. Since the amount of APE1 is much more abundant than pol $\beta$ in cells, the results indicated that APE1 3'-5' exonuclease cooperated with pol $\beta$ to serve as a proofreading factor of pol $\beta$ improving the fidelity of the polymerase during BER and preventing pol $\beta$-mediated mutagenesis. In Chapter 3, we further explored how bromate and chromate-induced oxidative DNA base lesions may modulate DNA methylation pattern at the tumor suppressor, BRCA1 gene via BER. We found that bromate and chromate-induced oxidative base lesions resulted in loss of $5 \mathrm{mCs}$ and mutations in the promoter and encoding regions of BRCA1 gene in HEK293 cells. Surprisingly, we found that bromate and chromate also induced additional DNA methylations at the CpGs adjacent to the CpGs bearing loss of $5 \mathrm{mCs}$ and mutations. Further mutational analysis on the mutation spectrum showed that bromate and chromate predominantly resulted in $\mathrm{C}$ to $\mathrm{G}$ and $\mathrm{C}$ to $\mathrm{A}$ mutations at the $\mathrm{CpGs}$ located at the BRCA1 gene promoter and encoding region. Furthermore, employing immunofluorescence, we showed that bromate and chromate induced the formation of pol $\beta$ foci in the nucleus of HEK293 cells indicating that pol $\beta$ was recruited to the DNA damage sites induced by the DNA damaging agents. We further demonstrated that the $\mathrm{C}$ to $\mathrm{G}$ and $\mathrm{C}$ to $\mathrm{A}$ mutations were mediated by pol $\beta$ nucleotide misinsertion in bypassing an 8 oxoG on the complementary strand of the gene. Furthermore, employing coimmunoprecipitation, we discovered an interaction between pol $\beta$ and DNMT1 in HEK293 
cells upon bromate and chromate treatment. The results showed that pol $\beta$ and DNMT1 interacted immediately after cells were treated with the DNA damaging agents. Yet, the interaction disappeared after $24 \mathrm{~h}$ recovery from the DNA damaging agents. We suggest that bromate and chromate-induced oxidative DNA damage recruited pol $\beta$ which in turn interacts and recruits DNMT1 to the sites adjacent to the DNA damage sites. This subsequently may allow DNMT1 to create additional $5 \mathrm{mCs}$ at the $\mathrm{CpGs}$ located at BRCA1 gene promoter and encoding regions, thereby altering DNA methylation pattern of the gene. Our results provide new insights into the mechanisms by which DNA base lesions modulate epigenetic features via BER that plays a central role in mediating the interaction between the human genome and epigenome. 


\section{REFERENCES}

Ahmad, M.K., Amani, S., and Mahmood, R. (2014). Potassium bromate causes cell lysis and induces oxidative stress in human erythrocytes. Environ Toxicol 29, 138-145.

Ahmad, M.K., Naqshbandi, A., Fareed, M., and Mahmood, R. (2012). Oral administration of a nephrotoxic dose of potassium bromate, a food additive, alters renal redox and metabolic status and inhibits brush border membrane enzymes in rats. Food Chem 134, 980-985.

Ajarem, J., Altoom, N.G., Allam, A.A., Maodaa, S.N., Abdel-Maksoud, M.A., and Chow, B.K. (2016). Oral administration of potassium bromate induces neurobehavioral changes, alters cerebral neurotransmitters level and impairs brain tissue of swiss mice. Behav Brain Funct : BBF 12, 14.

Alabdulkareem, H., Pinchinat, T., Khan, S., Landers, A., Christos, P., Simmons, R., and Moo, T.A. (2017). The impact of molecular subtype on breast cancer recurrence in young women treated with contemporary adjuvant therapy. Breast J DOI: 10.1111/tbj.12853, 16.

Alexander, J., and Aaseth, J. (1995). Uptake of chromate in human red blood cells and isolated rat liver cells: the role of the anion carrier. Analyst 120, 931-933.

Bakhanashvili, M. (2001). Exonucleolytic proofreading by p53 protein. Eur J Biochem 268, 2047-2054.

Balakrishnan, L., and Bambara, R.A. (2013). Flap endonuclease 1. Annu Rev Biochem 82, 119-138.

Ballmaier, D., and Epe, B. (2006). DNA damage by bromate: mechanism and consequences. Toxicology 221, 166-171.

Barlow, J.H., Faryabi, R.B., Callen, E., Wong, N., Malhowski, A., Chen, H.T., GutierrezCruz, G., Sun, H.W., McKinnon, P., Wright, G., et al. (2013). Identification of early replicating fragile sites that contribute to genome instability. Cell 152, 620-632.

Barnes, D.E., and Lindahl, T. (2004). Repair and genetic consequences of endogenous DNA base damage in mammalian cells. Annu Rev Genet 38, 445-476.

Bassett, E., Vaisman, A., Tropea, K.A., McCall, C.M., Masutani, C., Hanaoka, F., and Chaney, S.G. (2002a). Frameshifts and deletions during in vitro translesion synthesis past Pt-DNA adducts by DNA polymerases beta and eta. DNA Repair 1, 1003-1016. 
Bassett, E., Vaisman, A., Tropea, K.A., McCall, C.M., Masutani, C., Hanaoka, F., and Chaney, S.G. (2002b). Frameshifts and deletions during in vitro translesion synthesis past Pt-DNA adducts by DNA polymerases beta and eta. DNA Repair 1, 1003-1016.

Batra, V.K., Shock, D.D., Prasad, R., Beard, W.A., Hou, E.W., Pedersen, L.C., Sayer, J.M., Yagi, H., Kumar, S., Jerina, D.M., et al. (2006). Structure of DNA polymerase beta with a benzo[c]phenanthrene diol epoxide-adducted template exhibits mutagenic features. Proc Natl Acad Sci U S A 103, 17231-17236.

Bayon, H. (1912). Epithelial proliferation induced by the injection of gasworks tar. Lancet $180,2$.

Beard, W.A., Shock, D.D., Batra, V.K., Pedersen, L.C., and Wilson, S.H. (2009). DNA polymerase beta substrate specificity: side chain modulation of the "A-rule". The J Biol Chem 284, 31680-31689.

Beard, W.A., Shock, D.D., Batra, V.K., Prasad, R., and Wilson, S.H. (2014). Substrateinduced DNA Polymerase beta Activation. J Biol Chem 289, 31411-31422.

Beard, W.A., and Wilson, S.H. (2006). Structure and mechanism of DNA polymerase Beta. Chem Rev 106, 361-382.

Beard, W.A., and Wilson, S.H. (2014). Structure and mechanism of DNA polymerase beta. Biochemistry-US 53, 2768-2780.

Becker, P.B., Ruppert, S., and Schutz, G. (1987). Genomic footprinting reveals cell typespecific DNA binding of ubiquitous factors. Cell 51, 435-443.

Bellacosa, A., and Drohat, A.C. (2015). Role of base excision repair in maintaining the genetic and epigenetic integrity of CpG sites. DNA Repair 32, 33-42.

Belmadoui, N., Boussicault, F., Guerra, M., Ravanat, J.L., Chatgilialoglu, C., and Cadet, J. (2010). Radiation-induced formation of purine 5',8-cyclonucleosides in isolated and cellular DNA: high stereospecificity and modulating effect of oxygen. Org Biomol Chem $8,3211-3219$.

Berger, S.L. (2002). Histone modifications in transcriptional regulation. Curr Opin Genet Dev 12, 142-148.

Bhutani, N., Burns, D.M., and Blau, H.M. (2011). DNA demethylation dynamics. Cell 146, 866-872.

Biade, S., Sobol, R.W., Wilson, S.H., and Matsumoto, Y. (1998a). Impairment of proliferating cell nuclear antigen-dependent apurinic/apyrimidinic site repair on linear DNA. J Biol Chem 273, 898-902. 
Biade, S., Sobol, R.W., Wilson, S.H., and Matsumoto, Y. (1998b). Impairment of proliferating cell nuclear antigen-dependent apurinic/apyrimidinic site repair on linear DNA. J Biol Chem 273, 898-902.

Bird, A. (1992). The essentials of DNA methylation. Cell 70, 5-8.

Bird, A. (2002). DNA methylation patterns and epigenetic memory. Genes Dev 16, 6-21.

Bird, A., Taggart, M., Frommer, M., Miller, O.J., and Macleod, D. (1985). A fraction of the mouse genome that is derived from islands of nonmethylated, CpG-rich DNA. Cell 40, 91-99.

Bird, A.P. (1986). CpG-rich islands and the function of DNA methylation. Nature 321, 209-213.

Bjelland, S., Eide, L., Time, R.W., Stote, R., Eftedal, I., Volden, G., and Seeberg, E. (1995). Oxidation of thymine to 5-formyluracil in DNA: mechanisms of formation, structural implications, and base excision by human cell free extracts. Biochemistry 34, 1475814764.

Bochtler, M., Kolano, A., and Xu, G.L. (2017). DNA demethylation pathways: Additional players and regulators. Bioessays 39, 1-13.

Bosviel, R., Garcia, S., Lavediaux, G., Michard, E., Dravers, M., Kwiatkowski, F., Bignon, Y.J., and Bernard-Gallon, D.J. (2012). BRCA1 promoter methylation in peripheral blood DNA was identified in sporadic breast cancer and controls. Cancer Epidemiol 36, e177182.

Braithwaite, D.K., and Ito, J. (1993). Compilation, alignment, and phylogenetic relationships of DNA polymerases. Nucleic Acids Res 21, 787-802.

Brooks, P.J. (2008). The 8,5'-cyclopurine-2'-deoxynucleosides: candidate neurodegenerative DNA lesions in xeroderma pigmentosum, and unique probes of transcription and nucleotide excision repair. DNA Repair 7, 1168-1179.

Brooks, P.J., Wise, D.S., Berry, D.A., Kosmoski, J.V., Smerdon, M.J., Somers, R.L., Mackie, H., Spoonde, A.Y., Ackerman, E.J., Coleman, K., et al. (2000). The oxidative DNA lesion 8,5'-(S)-cyclo-2'-deoxyadenosine is repaired by the nucleotide excision repair pathway and blocks gene expression in mammalian cells. J Biol Chem 275, 22355-22362.

Brooks, S.C., Adhikary, S., Rubinson, E.H., and Eichman, B.F. (2013). Recent advances in the structural mechanisms of DNA glycosylases. Biochim Biophys Acta 1834, 247-271. 
Burma, S., Chen, B.P., Murphy, M., Kurimasa, A., and Chen, D.J. (2001). ATM phosphorylates histone $\mathrm{H} 2 \mathrm{AX}$ in response to DNA double-strand breaks. The J Biol Chem 276, 42462-42467.

Cadet, J., Delatour, T., Douki, T., Gasparutto, D., Pouget, J.P., Ravanat, J.L., and Sauvaigo, S. (1999). Hydroxyl radicals and DNA base damage. Mutat Res 424, 9-21.

Cadet, J., Douki, T., Gasparutto, D., and Ravanat, J.L. (2003). Oxidative damage to DNA: formation, measurement and biochemical features. Mutat Res 531, 5-23.

Campbell, K.C. (2006). Bromate-induced ototoxicity. Toxicology 221, 205-211.

Canitrot, Y., Hoffmann, J.S., Calsou, P., Hayakawa, H., Salles, B., and Cazaux, C. (2000). Nucleotide excision repair DNA synthesis by excess DNA polymerase beta: a potential source of genetic instability in cancer cells. FASEB J 14, 1765-1774.

Casadevall, M., and Kortenkamp, A. (1994). The generation of apurinic/apyrimidinic sites in isolated DNA during the reduction of chromate by glutathione. Carcinogenesis 15, 407409.

Casadevall, M., and Kortenkamp, A. (1995). The formation of both apurinic/apyrimidinic sites and single-strand breaks by chromate and glutathione arises from attack by the same single reactive species and is dependent on molecular oxygen. Carcinogenesis 16, 805-809.

Cavanagh, J., Weinberg, H., Gold, A., Sangalah, R., Marbury, D., Glaze, W. (1992). Ozonation Byproducts: Identification of Bromohydrins from the Ozonation of Natural Waters with Enhanced Bromide levels. Environ Sci Technol 26, 5.

Chary, P., Beard, W.A., Wilson, S.H., and Lloyd, R.S. (2012a). DNA polymerase beta gapfilling translesion DNA synthesis. Chem Res Toxicol 25, 2744-2754.

Chary, P., Beard, W.A., Wilson, S.H., and Lloyd, R.S. (2012b). DNA polymerase beta gapfilling translesion DNA synthesis. Chem Res Toxicol 25, 2744-2754.

Chatgilialoglu, C., D'Angelantonio, M., Kciuk, G., and Bobrowski, K. (2011a). New insights into the reaction paths of hydroxyl radicals with 2'-deoxyguanosine. Chem Res Toxicol 24, 2200-2206.

Chatgilialoglu, C., Ferreri, C., Masi, A., Sansone, A., Terzidis, M.A., and Tsakos, M. (2014). A problem solving approach for the diastereoselective synthesis of (5'S)- and (5'R)5',8-cyclopurine lesions. Org Chem Front 1, 698-702.

Chatgilialoglu, C., Ferreri, C., and Terzidis, M.A. (2011b). Purine 5',8-cyclonucleoside lesions: chemistry and biology. Chem Soc Rev 40, 1368-1382. 
Chen, Z.X., and Riggs, A.D. (2011). DNA methylation and demethylation in mammals. J Biol Chem 286, 18347-18353.

Cheung, P., Allis, C.D., and Sassone-Corsi, P. (2000). Signaling to chromatin through histone modifications. Cell 103, 263-271.

Chiarle, R., Zhang, Y., Frock, R.L., Lewis, S.M., Molinie, B., Ho, Y.J., Myers, D.R., Choi, V.W., Compagno, M., Malkin, D.J., et al. (2011). Genome-wide translocation sequencing reveals mechanisms of chromosome breaks and rearrangements in B cells. Cell 147, 107119.

Chou, K.M., and Cheng, Y.C. (2002). An exonucleolytic activity of human apurinic/apyrimidinic endonuclease on 3' mispaired DNA. Nature 415, 655-659.

Chuang, L.S., Ian, H.I., Koh, T.W., Ng, H.H., Xu, G., and Li, B.F. (1997). Human DNA(cytosine-5) methyltransferase-PCNA complex as a target for p21WAF1. Science 277, 1996-2000.

Cistulli, C., Lavrik, O.I., Prasad, R., Hou, E., and Wilson, S.H. (2004). AP endonuclease and poly(ADP-ribose) polymerase-1 interact with the same base excision repair intermediate. DNA Repair (Amst) 3, 581-591.

Clark, S.J., Harrison, J., and Molloy, P.L. (1997). Sp1 binding is inhibited by (m) $\mathrm{Cp}(\mathrm{m}) \mathrm{CpG}$ methylation. Gene 195, 67-71.

Clark, S.L., Rodriguez, A.M., Snyder, R.R., Hankins, G.D., and Boehning, D. (2012). Structure-Function Of The Tumor Suppressor BRCA1. Comput Struct Biotechnol J 1.

Cortellino, S., Xu, J., Sannai, M., Moore, R., Caretti, E., Cigliano, A., Le Coz, M., Devarajan, K., Wessels, A., Soprano, D., et al. (2011). Thymine DNA glycosylase is essential for active DNA demethylation by linked deamination-base excision repair. Cell 146, 67-79.

Cousineau, I., Abaji, C., and Belmaaza, A. (2005). BRCA1 regulates RAD51 function in response to DNA damage and suppresses spontaneous sister chromatid replication slippage: implications for sister chromatid cohesion, genome stability, and carcinogenesis. Cancer Res 65, 11384-11391.

Das, R.S., Samaraweera, M., Morton, M., Gascon, J.A., and Basu, A.K. (2012). Stability of N-glycosidic bond of (5'S)-8,5'-cyclo-2'-deoxyguanosine. Chem Res Toxicol 25, 24512461.

Datta, A., and Jinks-Robertson, S. (1995). Association of increased spontaneous mutation rates with high levels of transcription in yeast. Science 268, 1616-1619. 
David, S.S., O'Shea, V.L., and Kundu, S. (2007). Base-excision repair of oxidative DNA damage. Nature 447, 941-950.

David, S.S., and Williams, S.D. (1998). Chemistry of Glycosylases and Endonucleases Involved in Base-Excision Repair. Chem Rev 98, 1221-1262.

Dianov, G.L., Prasad, R., Wilson, S.H., and Bohr, V.A. (1999). Role of DNA polymerase beta in the excision step of long patch mammalian base excision repair. J Biol Chem 274, 13741-13743.

Ding, N., Bonham, E.M., Hannon, B.E., Amick, T.R., Baylin, S.B., and O'Hagan, H.M. (2016). Mismatch repair proteins recruit DNA methyltransferase 1 to sites of oxidative DNA damage. J Mol Cell Biol 8, 244-254.

Dizdaroglu, M., Jaruga, P., and Rodriguez, H. (2001a). Identification and quantification of 8,5'-cyclo-2'-deoxy-adenosine in DNA by liquid chromatography/ mass spectrometry. Free Radic Biol Med 30, 774-784.

Dizdaroglu, M., Jaruga, P., and Rodriguez, H. (2001b). Measurement of 8-hydroxy-2'deoxyguanosine in DNA by high-performance liquid chromatography-mass spectrometry: comparison with measurement by gas chromatography-mass spectrometry. Nucleic Acids Res 29, E12.

Dobrovic, A., and Simpfendorfer, D. (1997). Methylation of the BRCA1 gene in sporadic breast cancer. Cancer Res 57, 3347-3350.

Dongmei, L., Zhiwei, W., Qi, Z., Fuyi, C., Yujuan, S., and Xiaodong, L. (2015). Drinking water toxicity study of the environmental contaminant--Bromate. Regul Toxicol Pharmacol: RTP 73, 802-810.

Drohat, A.C., and Coey, C.T. (2016b). Role of Base Excision "Repair" Enzymes in Erasing Epigenetic Marks from DNA. Chem Rev 116, 12711-12729.

Efrati, E., Tocco, G., Eritja, R., Wilson, S.H., and Goodman, M.F. (1997). Abasic translesion synthesis by DNA polymerase beta violates the "A-rule". Novel types of nucleotide incorporation by human DNA polymerase beta at an abasic lesion in different sequence contexts. J Biol Chem 272, 2559-2569.

Egger, G., Jeong, S., Escobar, S.G., Cortez, C.C., Li, T.W., Saito, Y., Yoo, C.B., Jones, P.A., and Liang, G. (2006). Identification of DNMT1 (DNA methyltransferase 1) hypomorphs in somatic knockouts suggests an essential role for DNMT1 in cell survival. Proc Natl Acad Sci U S A 103, 14080-14085.

Ehrlich, M. (2005). The controversial denouement of vertebrate DNA methylation research. Biochemistry (Mosc) 70, 568-575. 
Ehrlich, M., Gama-Sosa, M.A., Huang, L.H., Midgett, R.M., Kuo, K.C., McCune, R.A., and Gehrke, C. (1982). Amount and distribution of 5-methylcytosine in human DNA from different types of tissues of cells. Nucleic Acids Res 10, 2709-2721.

Esteller, M., Silva, J.M., Dominguez, G., Bonilla, F., Matias-Guiu, X., Lerma, E., Bussaglia, E., Prat, J., Harkes, I.C., Repasky, E.A., et al. (2000). Promoter hypermethylation and BRCA1 inactivation in sporadic breast and ovarian tumors. J Natl Cancer Inst 92, 564-569.

Fabbro, M., Savage, K., Hobson, K., Deans, A.J., Powell, S.N., McArthur, G.A., and Khanna, K.K. (2004). BRCA1-BARD1 complexes are required for p53Ser-15 phosphorylation and a G1/S arrest following ionizing radiation-induced DNA damage. J Biol Chem 279, 31251-31258.

Feng, L., Huang, J., and Chen, J. (2009). MERIT40 facilitates BRCA1 localization and DNA damage repair. Genes Dev 23, 719-728.

Fleming, A.M., Ding, Y., and Burrows, C.J. (2017). Oxidative DNA damage is epigenetic by regulating gene transcription via base excision repair. Proc Natl Acad Sci U S A 114, 2604-2609.

Fortini, P., Pascucci, B., Parlanti, E., Sobol, R.W., Wilson, S.H., and Dogliotti, E. (1998). Different DNA polymerases are involved in the short- and long-patch base excision repair in mammalian cells. Biochemistry 37, 3575-3580.

Friedberg, E.C., Lehmann, A.R., and Fuchs, R.P. (2005). Trading places: how do DNA polymerases switch during translesion DNA synthesis? Mol Cell 18, 499-505.

Friedberg, E.C., and Lindahl, T. (2004). Inroads into base excision repair II. The discovery of DNA glycosylases. "An N-glycosidase from Escherichia coli that releases free uracil from DNA containing deaminated cytosine residues," Proc. Nat. Acad. Sci. USA, 1974. DNA repair 3, 1532-1536; discussion 1531-1532.

Fromme, J.C., Banerjee, A., and Verdine, G.L. (2004). DNA glycosylase recognition and catalysis. Curr Opin Struct Biol 14, 43-49.

Fromme, J.C., and Verdine, G.L. (2004). Base excision repair. Adv Protein Chem 69, 141.

Frosina, G., Fortini, P., Rossi, O., Carrozzino, F., Raspaglio, G., Cox, L.S., Lane, D.P., Abbondandolo, A., and Dogliotti, E. (1996). Two pathways for base excision repair in mammalian cells. J Biol Chem 271, 9573-9578. 
Fujiki, H. (2014). Gist of Dr. Katsusaburo Yamagiwa's papers entitled "Experimental study on the pathogenesis of epithelial tumors" (I to VI reports). Cancer Sci 105, 143-149.

Furlan, D., Trapani, D., Berrino, E., Debernardi, C., Panero, M., Libera, L., Sahnane, N., Riva, C., Tibiletti, M.G., Sessa, F., et al. (2017). Oxidative DNA damage induces hypomethylation in a compromised base excision repair colorectal tumourigenesis. $\mathrm{Br} \mathrm{J}$ Cancer 116, 793-801.

Gao, M., Binks, S.P., Chipman, J.K., Levy, L.S., Braithwaite, R.A., and Brown, S.S. (1992). Induction of DNA strand breaks in peripheral lymphocytes by soluble chromium compounds. Hum Exp Toxicol 11, 77-82.

Gehring, M., Reik, W., and Henikoff, S. (2009). DNA demethylation by DNA repair. Trends Genet 25, 82-90.

Gong, Z., and Zhu, J.K. (2011). Active DNA demethylation by oxidation and repair. Cell Res $21,1649-1651$.

Greenberg, R.A., Sobhian, B., Pathania, S., Cantor, S.B., Nakatani, Y., and Livingston, D.M. (2006). Multifactorial contributions to an acute DNA damage response by BRCA1/BARD1-containing complexes. Genes Dev 20, 34-46.

Ha, K., Lee, G.E., Palii, S.S., Brown, K.D., Takeda, Y., Liu, K., Bhalla, K.N., and Robertson, K.D. (2011). Rapid and transient recruitment of DNMT1 to DNA double-strand breaks is mediated by its interaction with multiple components of the DNA damage response machinery. Hum Mol Genet 20, 126-140.

Harrington, J.J., and Lieber, M.R. (1994). The characterization of a mammalian DNA structure-specific endonuclease. EMBO J 13, 1235-1246.

Hartwell, L.H., and Kastan, M.B. (1994). Cell cycle control and cancer. Science 266, 18211828.

Hashim, M.F., Schnetz-Boutaud, N., and Marnett, L.J. (1997a). Replication of templateprimers containing propanodeoxyguanosine by DNA polymerase beta. Induction of base pair substitution and frameshift mutations by template slippage and deoxynucleoside triphosphate stabilization. J Biol Chem 272, 20205-20212.

Hashim, M.F., Schnetz-Boutaud, N., and Marnett, L.J. (1997b). Replication of templateprimers containing propanodeoxyguanosine by DNA polymerase beta. Induction of base pair substitution and frameshift mutations by template slippage and deoxynucleoside triphosphate stabilization. J Biol Chem 272, 20205-20212. 
He, Y.F., Li, B.Z., Li, Z., Liu, P., Wang, Y., Tang, Q., Ding, J., Jia, Y., Chen, Z., Li, L., et al. (2011). Tet-mediated formation of 5-carboxylcytosine and its excision by TDG in mammalian DNA. Science 333, 1303-1307.

Henderson, B.R. (2012). The BRCA1 Breast Cancer Suppressor: Regulation of Transport, Dynamics, and Function at Multiple Subcellular Locations. Scientifica 2012, 796808.

Hendrich, B., and Bird, A. (1998). Identification and characterization of a family of mammalian methyl-CpG binding proteins. Mol Cell Biol 18, 6538-6547.

Herman, J.G., and Baylin, S.B. (2003). Gene silencing in cancer in association with promoter hypermethylation. N Engl J Med 349, 2042-2054.

Hodges, N.J., Adam, B., Lee, A.J., Cross, H.J., and Chipman, J.K. (2001). Induction of DNA-strand breaks in human peripheral blood lymphocytes and A549 lung cells by sodium dichromate: association with 8-oxo-2-deoxyguanosine formation and inter-individual variability. Mutagenesis 16, 467-474.

Hoeijmakers, J.H. (2001). Genome maintenance mechanisms for preventing cancer. Nature $411,366-374$.

Hoffmann, J.S., Pillaire, M.J., Garcia-Estefania, D., Lapalu, S., and Villani, G. (1996a). In vitro bypass replication of the cisplatin-d(GpG) lesion by calf thymus DNA polymerase beta and human immunodeficiency virus type I reverse transcriptase is highly mutagenic. J Biol Chem 271, 15386-15392.

Hoffmann, J.S., Pillaire, M.J., Garcia-Estefania, D., Lapalu, S., and Villani, G. (1996b). In vitro bypass replication of the cisplatin-d(GpG) lesion by calf thymus DNA polymerase beta and human immunodeficiency virus type I reverse transcriptase is highly mutagenic. J Biol Chem 271, 15386-15392.

Horton, J.K., Baker, A., Berg, B.J., Sobol, R.W., and Wilson, S.H. (2002). Involvement of DNA polymerase beta in protection against the cytotoxicity of oxidative DNA damage. DNA Repair 1,317-333.

Hu, Y.F., Hao, Z.L., and Li, R. (1999). Chromatin remodeling and activation of chromosomal DNA replication by an acidic transcriptional activation domain from BRCA1. Genes Dev 13, 637-642.

Huen, M.S., Grant, R., Manke, I., Minn, K., Yu, X., Yaffe, M.B., and Chen, J. (2007). RNF8 transduces the DNA-damage signal via histone ubiquitylation and checkpoint protein assembly. Cell 131, 901-914.

Huen, M.S., Sy, S.M., and Chen, J. (2010). BRCA1 and its toolbox for the maintenance of genome integrity. Nat Rev Mol Cell Biol 11, 138-148. 
Illingworth, R., Kerr, A., Desousa, D., Jorgensen, H., Ellis, P., Stalker, J., Jackson, D., Clee, C., Plumb, R., Rogers, J., et al. (2008). A novel CpG island set identifies tissuespecific methylation at developmental gene loci. PLoS Biol 6, e22.

Ito, S., and Kuraoka, I. (2015). Epigenetic modifications in DNA could mimic oxidative DNA damage: A double-edged sword. DNA Repair 32, 52-57.

Ito, S., Shen, L., Dai, Q., Wu, S.C., Collins, L.B., Swenberg, J.A., He, C., and Zhang, Y. (2011). Tet proteins can convert 5-methylcytosine to 5-formylcytosine and 5carboxylcytosine. Science 333, 1300-1303.

Jaruga, P., and Dizdaroglu, M. (2008). 8,5'-Cyclopurine-2'-deoxynucleosides in DNA: mechanisms of formation, measurement, repair and biological effects. DNA Repair 7, $1413-1425$.

Jennette, K.W. (1979). Chromate metabolism in liver microsomes. Biol Trace Elem Res 1, $55-62$.

Jiang, Z., Xu, M., Lai, Y., Laverde, E.E., Terzidis, M.A., Masi, A., Chatgilialoglu, C., and Liu, Y. (2015). Bypass of a 5',8-cyclopurine-2'-deoxynucleoside by DNA polymerase beta during DNA replication and base excision repair leads to nucleotide misinsertions and DNA strand breaks. DNA Repair 33, 24-34.

Jones, P.A. (2012). Functions of DNA methylation: islands, start sites, gene bodies and beyond. Nat Rev Genet 13, 484-492.

Jones, P.A., and Baylin, S.B. (2002). The fundamental role of epigenetic events in cancer. Nat Rev Genet 3, 415-428.

Jones, P.A., and Baylin, S.B. (2007). The epigenomics of cancer. Cell 128, 683-692.

Jones, P.A., and Takai, D. (2001). The role of DNA methylation in mammalian epigenetics. Science 293, 1068-1070.

Kamakura, N., Yamamoto, J., Brooks, P.J., Iwai, S., and Kuraoka, I. (2012). Effects of 5',8cyclodeoxyadenosine triphosphates on DNA synthesis. Chem Res Toxicol 25, 2718-2724.

Kareta, M.S., Botello, Z.M., Ennis, J.J., Chou, C., and Chedin, F. (2006). Reconstitution and mechanism of the stimulation of de novo methylation by human DNMT3L. J Biol Chem 281, 25893-25902.

Karwowski, B.T., Bellon, S., O'Neill, P., Lomax, M.E., and Cadet, J. (2014). Effects of (5'S)-5',8-cyclo-2'-deoxyadenosine on the base excision repair of oxidatively generated 
clustered DNA damage. A biochemical and theoretical study. Org Biomol Chem 12, 86718682.

Kass, S.U., Landsberger, N., and Wolffe, A.P. (1997). DNA methylation directs a timedependent repression of transcription initiation. Curr Biol 7, 157-165.

Kawanishi, S., Hiraku, Y., Murata, M., and Oikawa, S. (2002). The role of metals in sitespecific DNA damage with reference to carcinogenesis. Free Radic Biol Med 32, 822-832.

Kawanishi, S., Hiraku, Y., and Oikawa, S. (2001). Mechanism of guanine-specific DNA damage by oxidative stress and its role in carcinogenesis and aging. Mutat Res 488, 65-76.

Kawanishi, S., and Murata, M. (2006). Mechanism of DNA damage induced by bromate differs from general types of oxidative stress. Toxicology 221, 172-178.

Khan, N., Sharma, S., and Sultana, S. (2004). Attenuation of potassium bromate-induced nephrotoxicity by coumarin (1,2-benzopyrone) in Wistar rats: chemoprevention against free radical-mediated renal oxidative stress and tumor promotion response. Redox Rep 9, $19-28$.

Kim, H., Chen, J., and Yu, X. (2007). Ubiquitin-binding protein RAP80 mediates BRCA1dependent DNA damage response. Science 316, 1202-1205.

Kirkali, G., de Souza-Pinto, N.C., Jaruga, P., Bohr, V.A., and Dizdaroglu, M. (2009). Accumulation of (5'S)-8,5'-cyclo-2'-deoxyadenosine in organs of Cockayne syndrome complementation group B gene knockout mice. DNA Repair 8, 274-278.

Kirkali, G., Tunca, M., Genc, S., Jaruga, P., and Dizdaroglu, M. (2008). Oxidative DNA damage in polymorphonuclear leukocytes of patients with familial Mediterranean fever. Free Radic Biol Med 44, 386-393.

Klein, I.A., Resch, W., Jankovic, M., Oliveira, T., Yamane, A., Nakahashi, H., Di Virgilio, M., Bothmer, A., Nussenzweig, A., Robbiani, D.F., et al. (2011). Translocation-capture sequencing reveals the extent and nature of chromosomal rearrangements in $\mathrm{B}$ lymphocytes. Cell 147, 95-106.

Klungland, A., and Lindahl, T. (1997). Second pathway for completion of human DNA base excision-repair: reconstitution with purified proteins and requirement for DNase IV (FEN1). EMBO J 16, 3341-3348.

Kolas, N.K., Chapman, J.R., Nakada, S., Ylanko, J., Chahwan, R., Sweeney, F.D., Panier, S., Mendez, M., Wildenhain, J., Thomson, T.M., et al. (2007). Orchestration of the DNAdamage response by the RNF8 ubiquitin ligase. Science 318, 1637-1640. 
Kote-Jarai, Z., and Eeles, R.A. (1999). BRCA1, BRCA2 and their possible function in DNA damage response. Br J Cancer 81, 1099-1102.

Kropachev, K., Ding, S., Terzidis, M.A., Masi, A., Liu, Z., Cai, Y., Kolbanovskiy, M., Chatgilialoglu, C., Broyde, S., Geacintov, N.E., et al. (2014). Structural basis for the recognition of diastereomeric 5',8-cyclo-2'-deoxypurine lesions by the human nucleotide excision repair system. Nucleic Acids Res 42, 5020-5032.

Kuraoka, I., Bender, C., Romieu, A., Cadet, J., Wood, R.D., and Lindahl, T. (2000). Removal of oxygen free-radical-induced 5',8-purine cyclodeoxynucleosides from DNA by the nucleotide excision-repair pathway in human cells. Proc Natl Acad Sci U S A 97, 38323837.

Kuraoka, I., Robins, P., Masutani, C., Hanaoka, F., Gasparutto, D., Cadet, J., Wood, R.D., and Lindahl, T. (2001). Oxygen free radical damage to DNA. Translesion synthesis by human DNA polymerase eta and resistance to exonuclease action at cyclopurine deoxynucleoside residues. J Biol Chem 276, 49283-49288.

Kurokawa, Y., Aoki, S., Matsushima, Y., Takamura, N., Imazawa, T., and Hayashi, Y. (1986). Dose-response studies on the carcinogenicity of potassium bromate in F344 rats after long-term oral administration. J Natl Cancer Inst 77, 977-982.

Lai, Y., Budworth, H., Beaver, J.M., Chan, N.L., Zhang, Z., McMurray, C.T., and Liu, Y. (2016). Crosstalk between MSH2-MSH3 and polbeta promotes trinucleotide repeat expansion during base excision repair. Nat Commun 7, 12465.

Lan, L., Nakajima, S., Oohata, Y., Takao, M., Okano, S., Masutani, M., Wilson, S.H., and Yasui, A. (2004). In situ analysis of repair processes for oxidative DNA damage in mammalian cells. Proc Natl Acad Sci U S A 101, 13738-13743.

Lee, B., Morano, A., Porcellini, A., and Muller, M.T. (2012). GADD45alpha inhibition of DNMT1 dependent DNA methylation during homology directed DNA repair. Nucleic Acids Res 40, 2481-2493.

Leonhardt, H., Page, A.W., Weier, H.U., and Bestor, T.H. (1992). A targeting sequence directs DNA methyltransferase to sites of DNA replication in mammalian nuclei. Cell 71, 865-873.

Lewis, J.D., Meehan, R.R., Henzel, W.J., Maurer-Fogy, I., Jeppesen, P., Klein, F., and Bird, A. (1992). Purification, sequence, and cellular localization of a novel chromosomal protein that binds to methylated DNA. Cell 69, 905-914.

Li, E., Bestor, T.H., and Jaenisch, R. (1992). Targeted mutation of the DNA methyltransferase gene results in embryonic lethality. Cell 69, 915-926. 
Li, J., Braganza, A., and Sobol, R.W. (2013). Base excision repair facilitates a functional relationship between Guanine oxidation and histone demethylation. Antioxid Redox Signal $18,2429-2443$.

Li, Y.Q., Zhou, P.Z., Zheng, X.D., Walsh, C.P., and Xu, G.L. (2007). Association of Dnmt3a and thymine DNA glycosylase links DNA methylation with base-excision repair. Nucleic Acids Res 35, 390-400.

Lindahl, T., and Barnes, D.E. (2000). Repair of endogenous DNA damage. Cold Spring Harb Symp Quant Biol 65, 127-133.

Lindahl, T., and Wood, R.D. (1999). Quality control by DNA repair. Science 286, 18971905.

Liu, X., Holstege, H., van der Gulden, H., Treur-Mulder, M., Zevenhoven, J., Velds, A., Kerkhoven, R.M., van Vliet, M.H., Wessels, L.F., Peterse, J.L., et al. (2007a). Somatic loss of BRCA1 and p53 in mice induces mammary tumors with features of human BRCA1mutated basal-like breast cancer. Proc Natl Acad Sci U S A 104, 12111-12116.

Liu, Y., Beard, W.A., Shock, D.D., Prasad, R., Hou, E.W., and Wilson, S.H. (2005). DNA polymerase beta and flap endonuclease 1 enzymatic specificities sustain DNA synthesis for long patch base excision repair. J Biol Chem 280, 3665-3674.

Liu, Y., Kao, H.I., and Bambara, R.A. (2004). Flap endonuclease 1: a central component of DNA metabolism. Annu Rev Biochem 73, 589-615.

Liu, Y., Prasad, R., Beard, W.A., Kedar, P.S., Hou, E.W., Shock, D.D., and Wilson, S.H. (2007b). Coordination of steps in single-nucleotide base excision repair mediated by apurinic/apyrimidinic endonuclease 1 and DNA polymerase beta. J Biol Chem 282, 1353213541.

Liu, Y., and Wilson, S.H. (2012). DNA base excision repair: a mechanism of trinucleotide repeat expansion. Trends Biochem Sci 37, 162-172.

Liu, Z., Wu, J., and Yu, X. (2007c). CCDC98 targets BRCA1 to DNA damage sites. Nat Struct Mol Biol 14, 716-720.

Lodato, M.A., Woodworth, M.B., Lee, S., Evrony, G.D., Mehta, B.K., Karger, A., Lee, S., Chittenden, T.W., D'Gama, A.M., Cai, X., et al. (2015). Somatic mutation in single human neurons tracks developmental and transcriptional history. Science 350, 94-98.

Long, D.T., and Walter, J.C. (2012). A novel function for BRCA1 in crosslink repair. Mol Cell 46, 111-112. 
Lutsenko, E., and Bhagwat, A.S. (1999). Principal causes of hot spots for cytosine to thymine mutations at sites of cytosine methylation in growing cells. A model, its experimental support and implications. Mutat Res 437, 11-20.

Maga, G., van Loon, B., Crespan, E., Villani, G., and Hubscher, U. (2009a). The block of DNA polymerase delta strand displacement activity by an abasic site can be rescued by the concerted action of DNA polymerase beta and Flap endonuclease 1. J Biol Chem 284, 14267-14275.

Maga, G., van Loon, B., Crespan, E., Villani, G., and Hubscher, U. (2009b). The block of DNA polymerase delta strand displacement activity by an abasic site can be rescued by the concerted action of DNA polymerase beta and Flap endonuclease 1. J Biol Chem 284, $14267-14275$.

Mahfoudhi, E., Talhaoui, I., Cabagnols, X., Della Valle, V., Secardin, L., Rameau, P., Bernard, O.A., Ishchenko, A.A., Abbes, S., Vainchenker, W., et al. (2016). TET2-mediated 5-hydroxymethylcytosine induces genetic instability and mutagenesis. DNA Repair 43, 7888.

Mailand, N., Bekker-Jensen, S., Faustrup, H., Melander, F., Bartek, J., Lukas, C., and Lukas, J. (2007). RNF8 ubiquitylates histones at DNA double-strand breaks and promotes assembly of repair proteins. Cell 131, 887-900.

Maiti, A., and Drohat, A.C. (2011). Thymine DNA glycosylase can rapidly excise 5formylcytosine and 5-carboxylcytosine: potential implications for active demethylation of CpG sites. J Biol Chem 286, 35334-35338.

Maiuri, A.R., Peng, M., Sriramkumar, S., Kamplain, C.M., DeStefano Shields, C.E., Sears, C.L., and O'Hagan, H.M. (2017). Mismatch Repair Proteins Initiate Epigenetic Alterations during Inflammation-Driven Tumorigenesis. Cancer Res 77, 3467-3478.

Marietta, C., Gulam, H., and Brooks, P.J. (2002). A single 8,5'-cyclo-2'-deoxyadenosine lesion in a TATA box prevents binding of the TATA binding protein and strongly reduces transcription in vivo. DNA Repair 1, 967-975.

Masaoka, A., Matsubara, M., Hasegawa, R., Tanaka, T., Kurisu, S., Terato, H., Ohyama, Y., Karino, N., Matsuda, A., and Ide, H. (2003). Mammalian 5-formyluracil-DNA glycosylase. 2. Role of SMUG1 uracil-DNA glycosylase in repair of 5-formyluracil and other oxidized and deaminated base lesions. Biochemistry 42, 5003-5012.

Masuda, Y., Bennett, R.A., and Demple, B. (1998). Dynamics of the interaction of human apurinic endonuclease (Ape1) with its substrate and product. J Biol Chem 273, 3035230359. 
Matros, E., Wang, Z.C., Lodeiro, G., Miron, A., Iglehart, J.D., and Richardson, A.L. (2005). BRCA1 promoter methylation in sporadic breast tumors: relationship to gene expression profiles. Breast Cancer Res Treat 91, 179-186.

Meehan, R.R., Lewis, J.D., McKay, S., Kleiner, E.L., and Bird, A.P. (1989). Identification of a mammalian protein that binds specifically to DNA containing methylated CpGs. Cell $58,499-507$.

Messer, J., Reynolds, M., Stoddard, L., and Zhitkovich, A. (2006). Causes of DNA singlestrand breaks during reduction of chromate by glutathione in vitro and in cells. Free Radic. Biol. Med 40, 1981-1992.

Miki, Y., Swensen, J., Shattuck-Eidens, D., Futreal, P.A., Harshman, K., Tavtigian, S., Liu, Q., Cochran, C., Bennett, L.M., Ding, W., et al. (1994). A strong candidate for the breast and ovarian cancer susceptibility gene BRCA1. Science 266, 66-71.

Mitra, D., Luo, X., Morgan, A., Wang, J., Hoang, M.P., Lo, J., Guerrero, C.R., Lennerz, J.K., Mihm, M.C., Wargo, J.A., et al. (2012). An ultraviolet-radiation-independent pathway to melanoma carcinogenesis in the red hair/fair skin background. Nature 491, 449453.

Morgan, H.D., Dean, W., Coker, H.A., Reik, W., and Petersen-Mahrt, S.K. (2004). Activation-induced cytidine deaminase deaminates 5-methylcytosine in DNA and is expressed in pluripotent tissues: implications for epigenetic reprogramming. J Biol Chem $279,52353-52360$.

Mortusewicz, O., Schermelleh, L., Walter, J., Cardoso, M.C., and Leonhardt, H. (2005). Recruitment of DNA methyltransferase I to DNA repair sites. Proc Natl Acad Sci U S A 102, 8905-8909.

Moynahan, M.E., Chiu, J.W., Koller, B.H., and Jasin, M. (1999). Brcal controls homologydirected DNA repair. Mol. Cell 4, 511-518.

Murante, R.S., Rust, L., and Bambara, R.A. (1995). Calf 5' to 3' exo/endonuclease must slide from a 5 ' end of the substrate to perform structure-specific cleavage. J Biol Chem 270, 30377-30383.

Murata, M., Bansho, Y., Inoue, S., Ito, K., Ohnishi, S., Midorikawa, K., and Kawanishi, S. (2001). Requirement of glutathione and cysteine in guanine-specific oxidation of DNA by carcinogenic potassium bromate. Chem Res Toxicol 14, 678-685.

Neddermann, P., and Jiricny, J. (1993). The purification of a mismatch-specific thymineDNA glycosylase from HeLa cells. J Biol Chem 268, 21218-21224. 
Neddermann, P., and Jiricny, J. (1994). Efficient removal of uracil from G.U mispairs by the mismatch-specific thymine DNA glycosylase from HeLa cells. Proc Natl Acad Sci U S A

Neeley, W.L., and Essigmann, J.M. (2006). Mechanisms of formation, genotoxicity, and mutation of guanine oxidation products. Chemical research in toxicology 19, 491-505.

Niehrs, C. (2009). Active DNA demethylation and DNA repair. Differentiation 77, 1-11.

Niehrs, C., and Schafer, A. (2012). Active DNA demethylation by Gadd45 and DNA repair. Trends Cell Biol 22, 220-227.

O'Connell, M.J., Walworth, N.C., and Carr, A.M. (2000). The G2-phase DNA-damage checkpoint. Trends Cell Biol 10, 296-303.

O'Hagan, H.M., Wang, W., Sen, S., Destefano Shields, C., Lee, S.S., Zhang, Y.W., Clements, E.G., Cai, Y., Van Neste, L., Easwaran, H., et al. (2011). Oxidative damage targets complexes containing DNA methyltransferases, SIRT1, and polycomb members to promoter CpG Islands. Cancer Cell 20, 606-619.

Occupational, S., and Health Administration, D.o.L. (2006). Occupational exposure to hexavalent chromium. Final rule. Fed Regist 71, 10099-10385.

Okano, M., Bell, D.W., Haber, D.A., and Li, E. (1999). DNA methyltransferases Dnmt3a and Dnmt3b are essential for de novo methylation and mammalian development. Cell 99, 247-257.

Parikh, S.S., Mol, C.D., Slupphaug, G., Bharati, S., Krokan, H.E., and Tainer, J.A. (1998). Base excision repair initiation revealed by crystal structures and binding kinetics of human uracil-DNA glycosylase with DNA. The EMBO J 17, 5214-5226.

Parsons, J.L., Dianova, II, and Dianov, G.L. (2005). APE1-dependent repair of DNA single-strand breaks containing 3'-end 8-oxoguanine. Nucleic Acids Res 33, 2204-2209.

Pednekar, V., Weerasooriya, S., Jasti, V.P., and Basu, A.K. (2014). Mutagenicity and genotoxicity of (5'S)-8,5'-cyclo-2'-deoxyadenosine in Escherichia coli and replication of (5'S)-8,5'-cyclopurine-2'-deoxynucleosides in vitro by DNA polymerase IV, exo-free Klenow fragment, and Dpo4. Chem Res Toxicol 27, 200-210.

Perry, J.A., and Kornbluth, S. (2007). Cdc25 and Wee1: analogous opposites? Cell Div 2, 12.

Podlutsky, A.J., Dianova, II, Podust, V.N., Bohr, V.A., and Dianov, G.L. (2001). Human DNA polymerase beta initiates DNA synthesis during long-patch repair of reduced AP sites in DNA. EMBO J 20, 1477-1482. 
Prasad, R., Dianov, G.L., Bohr, V.A., and Wilson, S.H. (2000). FEN1 stimulation of DNA polymerase beta mediates an excision step in mammalian long patch base excision repair. J Biol Chem 275, 4460-4466.

Quievryn, G., Messer, J., and Zhitkovich, A. (2002). Carcinogenic chromium(VI) induces cross-linking of vitamin C to DNA in vitro and in human lung A549 cells. Biochemistry 41, 3156-3167.

Quievryn, G., Peterson, E., Messer, J., and Zhitkovich, A. (2003). Genotoxicity and mutagenicity of chromium(VI)/ascorbate-generated DNA adducts in human and bacterial cells. Biochemistry 42, 1062-1070.

Rahav, G.a.B. (2013). The Role of P53 Exonuclease in Accuracy of DNA Synthesis and Sensitivity to Nucleotide Analogs in Various Compartments of Cells. In New Research Directions in DNA Repair, C. Chen, ed. (InTech), pp. 281-308.

Randerath, K., Zhou, G.D., Somers, R.L., Robbins, J.H., and Brooks, P.J. (2001). A 32Ppostlabeling assay for the oxidative DNA lesion 8,5'-cyclo-2'-deoxyadenosine in mammalian tissues: evidence that four type II I-compounds are dinucleotides containing the lesion in the 3' nucleotide. J Biol Chem 276, 36051-36057.

Rice, J.C., Massey-Brown, K.S., and Futscher, B.W. (1998). Aberrant methylation of the BRCA1 CpG island promoter is associated with decreased BRCA1 mRNA in sporadic breast cancer cells. Oncogene 17, 1807-1812.

Rice, J.C., Ozcelik, H., Maxeiner, P., Andrulis, I., and Futscher, B.W. (2000). Methylation of the BRCA1 promoter is associated with decreased BRCA1 mRNA levels in clinical breast cancer specimens. Carcinogenesis 21, 1761-1765.

Rideout, W.M., 3rd, Coetzee, G.A., Olumi, A.F., and Jones, P.A. (1990). 5-Methylcytosine as an endogenous mutagen in the human LDL receptor and p53 genes. Science 249, 12881290.

Riggs, A.D., and Xiong, Z. (2004). Methylation and epigenetic fidelity. Proc Natl Acad Sci U S A 101, 4-5.

Robertson, K.D. (2005). DNA methylation and human disease. Nat Rev Genet 6, 597-610.

Russo, G., Landi, R., Pezone, A., Morano, A., Zuchegna, C., Romano, A., Muller, M.T., Gottesman, M.E., Porcellini, A., and Avvedimento, E.V. (2016). DNA damage and Repair Modify DNA methylation and Chromatin Domain of the Targeted Locus: Mechanism of allele methylation polymorphism. Sci Rep 6, 33222. 
Salnikow, K., and Zhitkovich, A. (2008). Genetic and epigenetic mechanisms in metal carcinogenesis and cocarcinogenesis: nickel, arsenic, and chromium. Chem Res Toxicol $21,28-44$.

Sampath, H., McCullough, A.K., and Lloyd, R.S. (2012). Regulation of DNA glycosylases and their role in limiting disease. Free Radic Res 46, 460-478.

Sarni, D., and Kerem, B. (2016). The complex nature of fragile site plasticity and its importance in cancer. Curr Opin Cell Biol 40, 131-136.

Sassa, A., Beard, W.A., Prasad, R., and Wilson, S.H. (2012). DNA sequence context effects on the glycosylase activity of human 8-oxoguanine DNA glycosylase. J Biol Chem 287, 36702-36710.

Sassa, A., Caglayan, M., Dyrkheeva, N.S., Beard, W.A., and Wilson, S.H. (2014). Base excision repair of tandem modifications in a methylated $\mathrm{CpG}$ dinucleotide. J Biol Chem 289, 13996-14008.

Scanlon, K.J., Kashani-Sabet, M., and Miyachi, H. (1989). Differential gene expression in human cancer cells resistant to cisplatin. Cancer Invest 7, 581-587.

Scully, R., Chen, J., Plug, A., Xiao, Y., Weaver, D., Feunteun, J., Ashley, T., and Livingston, D.M. (1997). Association of BRCA1 with Rad51 in mitotic and meiotic cells. Cell 88, 265-275.

Seisenberger, S., Peat, J.R., Hore, T.A., Santos, F., Dean, W., and Reik, W. (2013). Reprogramming DNA methylation in the mammalian life cycle: building and breaking epigenetic barriers. Philos Trans R Soc Lond B Biol Sci 368, 20110330.

Servant, L., Cazaux, C., Bieth, A., Iwai, S., Hanaoka, F., and Hoffmann, J.S. (2002a). A role for DNA polymerase beta in mutagenic UV lesion bypass. J Biol Chem 277, 5004650053.

Servant, L., Cazaux, C., Bieth, A., Iwai, S., Hanaoka, F., and Hoffmann, J.S. (2002b). A role for DNA polymerase beta in mutagenic UV lesion bypass. J Biol Chem 277, 5004650053.

Shah, A.M., Maitra, M., and Sweasy, J.B. (2003). Variants of DNA polymerase Beta extend mispaired DNA due to increased affinity for nucleotide substrate. Biochemistry 42 , 10709-10717.

Shaked, H., Hofseth, L.J., Chumanevich, A., Chumanevich, A.A., Wang, J., Wang, Y., Taniguchi, K., Guma, M., Shenouda, S., Clevers, H., et al. (2012). Chronic epithelial NFkappaB activation accelerates APC loss and intestinal tumor initiation through iNOS upregulation. Proc Natl Acad Sci U S A 109, 14007-14012. 
Shao, G., Patterson-Fortin, J., Messick, T.E., Feng, D., Shanbhag, N., Wang, Y., and Greenberg, R.A. (2009). MERIT40 controls BRCA1-Rap80 complex integrity and recruitment to DNA double-strand breaks. Genes Dev 23, 740-754.

Siegfried, Z., and Cedar, H. (1997). DNA methylation: a molecular lock. Curr Biol: CB 7, R305-307.

Slade, P.G., Hailer, M.K., Martin, B.D., and Sugden, K.D. (2005). Guanine-specific oxidation of double-stranded DNA by $\mathrm{Cr}(\mathrm{VI})$ and ascorbic acid forms spiroiminodihydantoin and 8-oxo-2'-deoxyguanosine. Chem Res Toxicol 18, 1140-1149.

Smith, Z.D., and Meissner, A. (2013). DNA methylation: roles in mammalian development. Nat Rev Genet 14, 204-220.

Sobhian, B., Shao, G., Lilli, D.R., Culhane, A.C., Moreau, L.A., Xia, B., Livingston, D.M., and Greenberg, R.A. (2007). RAP80 targets BRCA1 to specific ubiquitin structures at DNA damage sites. Science 316, 1198-1202.

Srivastava, D.K., Husain, I., Arteaga, C.L., and Wilson, S.H. (1999). DNA polymerase beta expression differences in selected human tumors and cell lines. Carcinogenesis 20, 1049-1054.

Stearns, D.M., and Wetterhahn, K.E. (1994). Reaction of chromium(VI) with ascorbate produces chromium(V), chromium(IV), and carbon-based radicals. Chem Res Toxicol 7, 219-230.

Stevens, A.J., Guan, L., Bebenek, K., Kunkel, T.A., and Greenberg, M.M. (2013). DNA polymerase lambda inactivation by oxidized abasic sites. Biochemistry 52, 975-983.

Stirzaker, C., Millar, D.S., Paul, C.L., Warnecke, P.M., Harrison, J., Vincent, P.C., Frommer, M., and Clark, S.J. (1997). Extensive DNA methylation spanning the Rb promoter in retinoblastoma tumors. Cancer Res 57, 2229-2237.

Stucki, M., Clapperton, J.A., Mohammad, D., Yaffe, M.B., Smerdon, S.J., and Jackson, S.P. (2005). MDC1 directly binds phosphorylated histone H2AX to regulate cellular responses to DNA double-strand breaks. Cell 123, 1213-1226.

Subramaniam, D., Thombre, R., Dhar, A., and Anant, S. (2014). DNA methyltransferases: a novel target for prevention and therapy. Front Oncol 4, 80.

Sugiyama, M., Wang, X.W., and Costa, M. (1986). Comparison of DNA lesions and cytotoxicity induced by calcium chromate in human, mouse, and hamster cell lines. Cancer Res 46, 4547-4551. 
Sun, H., Zhou, X., Chen, H., Li, Q., and Costa, M. (2009). Modulation of histone methylation and MLH1 gene silencing by hexavalent chromium. Toxicol Appl Pharmacol 237, 258-266.

Suzuki, M.M., and Bird, A. (2008). DNA methylation landscapes: provocative insights from epigenomics. Nat Rev Genet 9, 465-476.

Tate, P., Skarnes, W., and Bird, A. (1996). The methyl-CpG binding protein MeCP2 is essential for embryonic development in the mouse. Nat Genet 12, 205-208.

Tate, P.H., and Bird, A.P. (1993). Effects of DNA methylation on DNA-binding proteins and gene expression. Curr Opin Genet Dev 3, 226-231.

Tilstra, J.S., Robinson, A.R., Wang, J., Gregg, S.Q., Clauson, C.L., Reay, D.P., Nasto, L.A., St Croix, C.M., Usas, A., Vo, N., et al. (2012). NF-kappaB inhibition delays DNA damage-induced senescence and aging in mice. J Clin Invest 122, 2601-2612.

Tomasetti, C., and Vogelstein, B. (2015). Cancer etiology. Variation in cancer risk among tissues can be explained by the number of stem cell divisions. Science 347, 78-81.

Tubbs, A., and Nussenzweig, A. (2017). Endogenous DNA Damage as a Source of Genomic Instability in Cancer. Cell 168, 644-656.

Ueno, S., Kashimoto, T., Susa, N., Furukawa, Y., Ishii, M., Yokoi, K., Yasuno, M., Sasaki, Y.F., Ueda, J., Nishimura, Y., et al. (2001). Detection of dichromate (VI)-induced DNA strand breaks and formation of paramagnetic chromium in multiple mouse organs. Toxicol Appl Pharmacol 170, 56-62.

Vaisman, A., and Chaney, S.G. (2000a). The efficiency and fidelity of translesion synthesis past cisplatin and oxaliplatin GpG adducts by human DNA polymerase beta. J Biol Chem $275,13017-13025$.

Vaisman, A., and Chaney, S.G. (2000b). The efficiency and fidelity of translesion synthesis past cisplatin and oxaliplatin GpG adducts by human DNA polymerase beta. J Biol Chem $275,13017-13025$.

Valinluck, V., and Sowers, L.C. (2007). Inflammation-mediated cytosine damage: a mechanistic link between inflammation and the epigenetic alterations in human cancers. Cancer Res 67, 5583-5586.

Villani, G., Hubscher, U., Gironis, N., Parkkinen, S., Pospiech, H., Shevelev, I., di Cicco, G., Markkanen, E., Syvaoja, J.E., and Tanguy Le Gac, N. (2011a). In vitro gap-directed translesion DNA synthesis of an abasic site involving human DNA polymerases epsilon, lambda, and beta. J Biol Chem 286, 32094-32104. 
Villani, G., Hubscher, U., Gironis, N., Parkkinen, S., Pospiech, H., Shevelev, I., di Cicco, G., Markkanen, E., Syvaoja, J.E., and Tanguy Le Gac, N. (2011b). In vitro gap-directed translesion DNA synthesis of an abasic site involving human DNA polymerases epsilon, lambda, and beta. J Biol Chem 286, 32094-32104.

Voet, D. and Voet, J.G. (2011). Biochemistry $4^{\text {th }}$ edition p94.

Voitkun, V., Zhitkovich, A., and Costa, M. (1994). Complexing of amino acids to DNA by chromate in intact cells. Environ Health Perspect 102 Suppl 3, 251-255.

Voitkun, V., Zhitkovich, A., and Costa, M. (1998). Cr(III)-mediated crosslinks of glutathione or amino acids to the DNA phosphate backbone are mutagenic in human cells. Nucleic Acids Res 26, 2024-2030.

Waldron, H.A. (1983a). A brief history of scrotal cancer. Br J Ind Med 40, 390-401. Waldron, H.A. (1983b). On the history of scrotal cancer. Ann R Coll Surg Engl 65, 420422.

Wallace, S.S. (2014). Base excision repair: a critical player in many games. DNA Repair 19, 14-26.

Wang, B., Hurov, K., Hofmann, K., and Elledge, S.J. (2009). NBA1, a new player in the Brcal A complex, is required for DNA damage resistance and checkpoint control. Genes Dev 23, 729-739.

Wang, B., Matsuoka, S., Ballif, B.A., Zhang, D., Smogorzewska, A., Gygi, S.P., and Elledge, S.J. (2007). Abraxas and RAP80 form a BRCA1 protein complex required for the DNA damage response. Science 316, 1194-1198.

Wang, J., Clauson, C.L., Robbins, P.D., Niedernhofer, L.J., and Wang, Y. (2012). The oxidative DNA lesions 8,5'-cyclopurines accumulate with aging in a tissue-specific manner. Aging cell 11, 714-716.

Wang, J., Yuan, B., Guerrero, C., Bahde, R., Gupta, S., and Wang, Y. (2011). Quantification of oxidative DNA lesions in tissues of Long-Evans Cinnamon rats by capillary high-performance liquid chromatography-tandem mass spectrometry coupled with stable isotope-dilution method. Anal Chem 83, 2201-2209.

Wang, Y. (2008). Bulky DNA lesions induced by reactive oxygen species. Chem Res Toxicol 21, 276-281.

Wei, M., Grushko, T.A., Dignam, J., Hagos, F., Nanda, R., Sveen, L., Xu, J., Fackenthal, J., Tretiakova, M., Das, S., et al. (2005). BRCA1 promoter methylation in sporadic breast cancer is associated with reduced BRCA1 copy number and chromosome 17 aneusomy. Cancer Res 65, 10692-10699. 
Williams, K., Christensen, J., Pedersen, M.T., Johansen, J.V., Cloos, P.A., Rappsilber, J., and Helin, K. (2011). TET1 and hydroxymethylcytosine in transcription and DNA methylation fidelity. Nature 473, 343-348.

Wilson, D.M., 3rd, Takeshita, M., Grollman, A.P., and Demple, B. (1995). Incision activity of human apurinic endonuclease (Ape) at abasic site analogs in DNA. J Biol Chem 270, 16002-16007.

Wilson, S.H., Beard, W.A., Shock, D.D., Batra, V.K., Cavanaugh, N.A., Prasad, R., Hou, E.W., Liu, Y., Asagoshi, K., Horton, J.K., et al. (2010). Base excision repair and design of small molecule inhibitors of human DNA polymerase beta. Cell Mol Life Sci 67, 36333647.

Wolfle, W.T., Washington, M.T., Prakash, L., and Prakash, S. (2003). Human DNA polymerase kappa uses template-primer misalignment as a novel means for extending mispaired termini and for generating single-base deletions. Genes Dev 17, 2191-2199.

Wooster, R., Bignell, G., Lancaster, J., Swift, S., Seal, S., Mangion, J., Collins, N., Gregory, S., Gumbs, C., and Micklem, G. (1995). Identification of the breast cancer susceptibility gene BRCA2. Nature 378, 789-792.

Wu, J., Huen, M.S., Lu, L.Y., Ye, L., Dou, Y., Ljungman, M., Chen, J., and Yu, X. (2009). Histone ubiquitination associates with BRCA1-dependent DNA damage response. Mol Cell Biol 29, 849-860.

Xu, B., Kim, S., and Kastan, M.B. (2001). Involvement of Brcal in S-phase and G(2)phase checkpoints after ionizing irradiation. Mol Cell Biol 21, 3445-3450.

Xu, M., Lai, Y., Jiang, Z., Terzidis, M.A., Masi, A., Chatgilialoglu, C., and Liu, Y. (2014). A 5', 8-cyclo-2'-deoxypurine lesion induces trinucleotide repeat deletion via a unique lesion bypass by DNA polymerase beta. Nucleic Acids Res 42, 13749-13763.

Yarden, R.I., Pardo-Reoyo, S., Sgagias, M., Cowan, K.H., and Brody, L.C. (2002). BRCA1 regulates the G2/M checkpoint by activating Chk1 kinase upon DNA damage. Nat Genet $30,285-289$.

You, C., Swanson, A.L., Dai, X., Yuan, B., Wang, J., and Wang, Y. (2013). Translesion synthesis of 8,5'-cyclopurine-2'-deoxynucleosides by DNA polymerases eta, iota, and zeta. J Biol Chem 288, 28548-28556.

Yuan, B., Wang, J., Cao, H., Sun, R., and Wang, Y. (2011). High-throughput analysis of the mutagenic and cytotoxic properties of DNA lesions by next-generation sequencing. Nucleic Acids Res 39, 5945-5954. 
Zhang, F., Fu, L., and Wang, Y. (2013). 6-thioguanine induces mitochondrial dysfunction and oxidative DNA damage in acute lymphoblastic leukemia cells. Mol Cell Proteomics $12,3803-3811$.

Zhang, L., Lu, X., Lu, J., Liang, H., Dai, Q., Xu, G.L., Luo, C., Jiang, H., and He, C. (2012). Thymine DNA glycosylase specifically recognizes 5-carboxylcytosine-modified DNA. Nat Chem Biol 8, 328-330.

Zhang, Y., Wu, X., Yuan, F., Xie, Z., and Wang, Z. (2001). Highly frequent frameshift DNA synthesis by human DNA polymerase mu. Mol Cell Biol 21, 7995-8006.

Zhao, G.Y., Sonoda, E., Barber, L.J., Oka, H., Murakawa, Y., Yamada, K., Ikura, T., Wang, X., Kobayashi, M., Yamamoto, K., et al. (2007). A critical role for the ubiquitinconjugating enzyme Ubc13 in initiating homologous recombination. Mol Cell 25, 663-675.

Zhao, H., Watkins, J.L., and Piwnica-Worms, H. (2002). Disruption of the checkpoint kinase $1 /$ cell division cycle $25 \mathrm{~A}$ pathway abrogates ionizing radiation-induced $\mathrm{S}$ and $\mathrm{G} 2$ checkpoints. Proc Natl Acad Sci U S A 99, 14795-14800.

Zhitkovich, A. (2005). Importance of chromium-DNA adducts in mutagenicity and toxicity of chromium(VI). Chem Res Toxicol 18, 3-11.

Zhitkovich, A., Voitkun, V., and Costa, M. (1995). Glutathione and free amino acids form stable complexes with DNA following exposure of intact mammalian cells to chromate. Carcinogenesis 16, 907-913.

Zhou, J., Ahn, J., Wilson, S.H., and Prives, C. (2001). A role for p53 in base excision repair. EMBO J 20, 914-923.

Zhou, X., Li, Q., Arita, A., Sun, H., and Costa, M. (2009). Effects of nickel, chromate, and arsenite on histone 3 lysine methylation. Toxicol Appl Pharmacol 236, 78-84.

Zhu, J.K. (2009). Active DNA demethylation mediated by DNA glycosylases. Annu Rev Genet 43, 143-166. 
VITA

ZHONGLIANG JIANG

2005-2009

B.S., Pharmacy

Heilongjiang University of Chinese Medicine

Harbin, Heilongjiang, P. R. China

2009-2012

M.S., Pharmacology

Sichuan University

Chengdu, Sichuan, P. R. China

2012-2017

$\mathrm{Ph} . \mathrm{D}$. Candidate, Biochemistry

Florida International University

Miami, Florida, USA

\section{PUBLICATIONS AND PRESENTATIONS}

Lai, Y., Jiang, Z., Zhou, J., Osemota, E., and Liu, Y. (2015) AP endonuclease 1 prevents the extension of a $\mathrm{T} / \mathrm{G}$ mismatch by DNA polymerase $\beta$ to prevent mutations in $\mathrm{CpGs}$ during base excision repair. DNA Repair 43:89-97.

Jiang, Z., Xu, M., Lai, Y., Laverde, E., Terzidis, M., Masi, A., Chatgilialoglu, C., and Liu, Y. (2014) Bypass of a 5',8-cyclopurine-2'-deoxynucleoside by DNA polymerase $\beta$ during DNA replication and base excision repair leads to nucleotide misinsertions and DNA strand breaks. DNA Repair 33: 24-34.

Xu, M., Lai, Y., Jiang, Z., Terzidis, M., Masi, A., Chatgilialoglu, C., and Liu, Y. (2014) A 5',8-cyclo-2'-deoxypurine lesion induces trinucleotide repeat deletion via a unique lesion bypass by DNA polymerase. Nucleic Acids Research 42: 13749-13763.

Jiang, Z., Beaver, J., Lai, Y., Rolle, S., Zamora, M., Rein, H., and Liu, Y., Environmentallyinduced oxidative DNA damage modulates DNA methylation pattern in human breast cancer 1 (BRCA1) gene via base excision repair, 19th Annual Biomedical and Comparative Immunology Symposium, Miami, FL., March 30-31, 2017 (Oral presentation).

Jiang, Z., Beaver, J., Lai, Y., Rolle, S., Zamora, M., Rein, H., and Liu, Y., Environmentallyinduced oxidative DNA damage disrupts DNA methylation pattern in human breast cancer 1 (BRCA1) gene via base excision repair, Graduate Student Appreciation Week 2017, Florida International University Scholarly Forum, Miami, FL., March 27-28, 2017 (Oral presentation). 
Jiang, Z., Beaver, J., Lai, Y., Rolle, S., Zamora, M., Rein, H., and Liu, Y., (2016) Environmentally-induced oxidative DNA damage disrupts DNA methylation pattern in human breast cancer type 1 (BRCA1) gene via base excision repair. $47^{\text {th }}$ Environmental Mutagenesis and Genomics Society Annual Meeting. Kansas City, Missouri, Sept. 24-28, 2016 (Oral presentation).

Jiang, Z., Beaver, J.M., Lai, Y., and Liu, Y. (2016) DNA base lesions and base excision repair modulate the epigenetic stability of breast cancer 1 (BRCA1) gene. 18th Annual Biomedical and Comparative Immunology Symposium. Miami, FL., March 26-27, 2016 (Oral presentation).

Jiang, Z., Beaver, J.M., Lai, Y., and Liu, Y. (2016) Epigenetic stability of breast cancer 1 (BRCA1) gene modulated by DNA base lesions and base excision repair. Florida International University Scholarly Forum. Miami, FL., March 28-29, 2016 (Oral presentation).

Jiang, Z., Xu, M., Lai, Y., Laverde, E., Terzidis, M., Masi, A., Chatgilialoglu, C., and Liu, Y. (2015) Bypass of a 5',8-deoxypurine lesion by DNA polymerase $\beta$ leads to nucleotide misinsertions and DNA strand breaks during DNA replication and base excision repair. Florida International University Scholarly Forum, Miami, FL., April 3-4, 2015 (Oral presentation).

Jiang, Z., Xu, M., Lai, Y., Laverde, E., Terzidis, M., Masi, A., Chatgilialoglu, C., and Liu, Y. (2015) Bypass of a 5',8-deoxypurine lesion by DNA polymerase $\beta$ leads to nucleotide misinsertions and DNA strand breaks during DNA replication and base excision repair.17th Annual Biomedical and Comparative Immunology Symposium, Miami, FL., March 26-27, 2015 (Oral presentation)

Jiang, Z., Xu, M., Lai, Y., Laverde, E., Terzidis, M., Masi, A., Chatgilialoglu, C., and Liu, Y. (2014)DNA polymerase $\beta$ plays a predominant role in bypassing a 5',8-deoxypurine lesion during DNA replication and DNA base excision repair, 45th Annual Environmental Mutagenesis and Genomic Society Conference, Orlando, FL., Sept. 12-17 (2014)(Poster presentation). 